\title{
REGRESSÃo LOGÍSTICA APLICADA NA IDENTIFICAÇÃO DE FATORES DE RISCO PARA DOENÇAS EM ANIMAIS DOMÉSTICOS
}

\author{
SILVANO CESAR DA COSTA \\ Licenciado em Matemática
}

Orientador: Prof. Dr. DÉCIO BARBIN

Dissertação apresentada à Escola Superior de Agricultura "Luiz de Queiroz", Universidade de São Paulo, para obtenção do título de Mestre em Agronomia. Área de Concentração: Estatística e Experimentação Agronômica.

P I R A C I C A B A

Estado de São Paulo - Brasil

Julho - 1997 
Dados Internacionais de Catalogação na Publicação (CIP)

DIVISÃo DE BIBLIOTECA E DOCUMENTAÇÃO - Campus "Luiz de Queiroz"/USP

\section{Costa, Silvano Cesar da}

Regressão logistica aplicada na identificação de fatores de risco para doenças em animais domésticos / Silvano Cesar da Costa. - - Piracicaba, 1997.

$104 \mathrm{p}$.

Dissertação (mestrado) - - Escola Superior de Agricultura Luiz de Queiroz, 1997. Bibliografia.

1. Estatistica aplicada 2. Método da máxima verossimilhança 3. Patologia animal 4. SAS (programa de computador) I. Título 


\section{REGRESSÃo LOGÍSTICA APLICADA NA IDENTIFICAÇÃO DE FATORES DE RISCO PARA DOENÇAS EM ANIMAIS DOMÉSTICOS}

Aprovado em: 05/09/1997

Comissão Julgadora:

Prof. Dr. Décio Barbin ESALQ/USP

Prof . Dra. Clarice Garcia Borges Demétrio ESALQ/USP

Prof ${ }^{\mathrm{a}}$. Dra. Tiemi Matsuo UEL

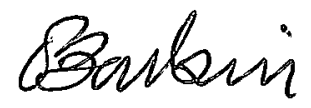

Prof. Dr. Décio Barbin Orientador 
A DEUS

Aos meus pais, JOÃO e MARIA (in memorian)

À minha esposa SIMONI

Dedico. 


\section{AGRADECIMENTOS}

- A DEUS, a capacidade, a inteligência e as oportunidades que me deu.

- Ao Professor Dr. Décio Barbin, a dedicação, a orientação, a amizade e o apoio nos momentos dificeis;

- À Prof $f^{a}$. Dra. Tiemi Matsuo, a sugestão do tema, a orientação, o incentivo e a amizade;

- Aos professores do DMAP - Departamento de Matemática Aplicada da Universidade Estadual de Londrina o incentivo;

- À familia Patriota: Afrânio, Mirian, Adriana, Emerson e Paulo o apoio e a paciência;

- À família Alves: Iza, Rosa e Diva, a acolhida e o carinho;

- Aos amigos Antonio Cláudio, Elizabeth Strapasson, Renata Totti e Rui Lyu Komi, os bons e maus momentos juntos. Os muitos fins de semana que passamos estudando, a amizade, o carinho e a confiança;

- Aos docentes do curso de Pós-Graduação em Estatística e Experimentação Agronômica da ESALQ-USP, o conhecimento e a experiência transmitidos;

- Aos funcionários do Departamento de Matemática e Estatística da ESALQ-USP, Expedita, Luciane, Malvina, Rosni e Solange a amizade e o apoio; 
- Ao Prof. Ms. Pedro Luiz de Camargo, do Hospital Veterinário da Universidade Estadual de Londrina, o fornecimento dos dados;

- À CAPES a concessão da bolsa;

- À bibliotecária Eliana Sabino, a revisão da referência bibliográfica;

- A todos, que de forma direta ou indireta, contribuíram para a realização deste trabalho. 


\section{SUMÁRIO}

RESUMO

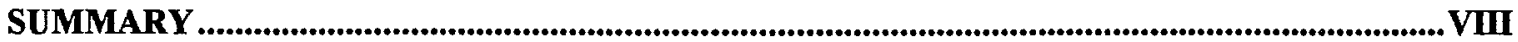

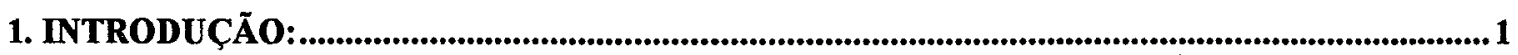

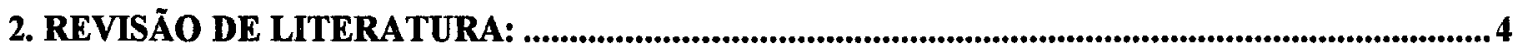

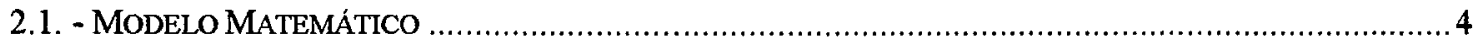

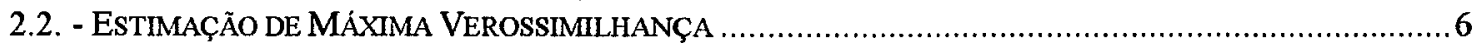

2.2.1. - Máxima Verossimilhança Incondicional .................................................................... 7

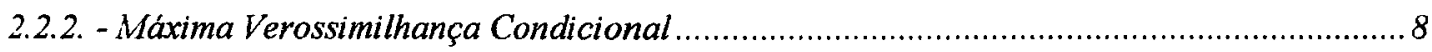

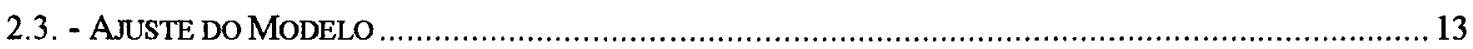

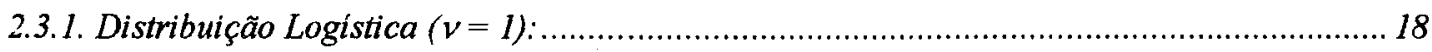

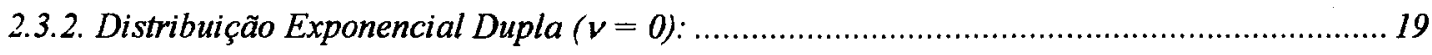

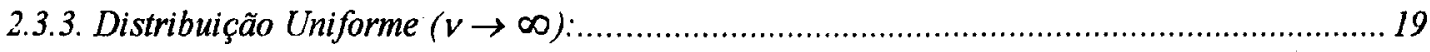

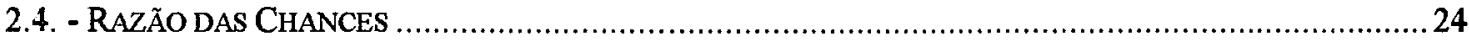

2.5. - "OUTLIERS"

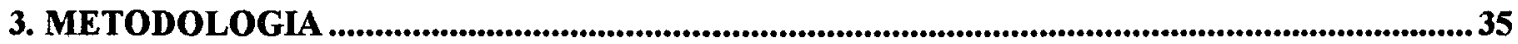

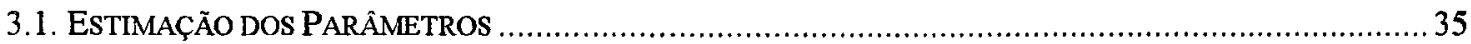

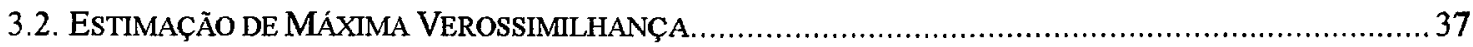

3.3. AJUSTE DE UM MODELo LINEAR GENERALIZAdo A DaDOs BINOMIAIS ...................................40

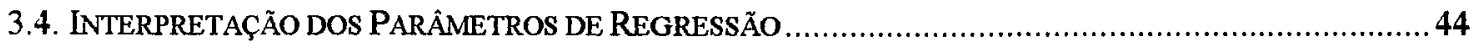

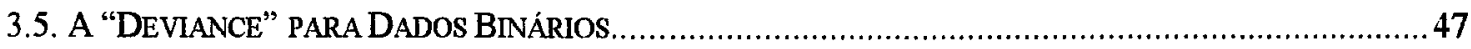

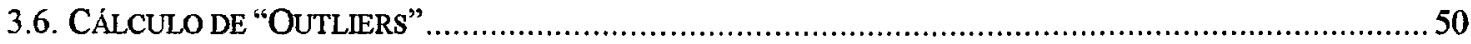

4. EXEMPLO DE APLICAÇÃO

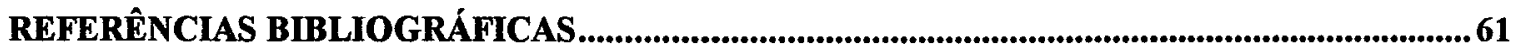

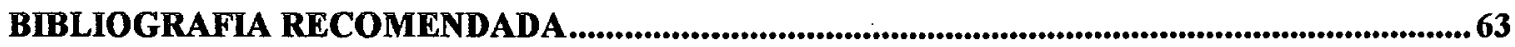

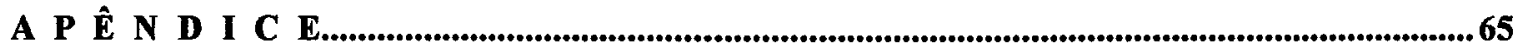




\title{
REGRESSÃo LOGÍSTICA APLICADA NA IDENTIFICAÇÃO DE FATORES DE RISCO PARA DOENÇAS EM ANIMAIS DOMÉSTICOS
}

\author{
Autor: SILVANO CESAR DA COSTA \\ Orientador: Prof. Dr. DÉCIO BARBIN
}

\section{RESUMO}

$\mathrm{Na}$ medicina animal, uma doença que acomete muitos cães é a Gastroenterite Hemorrágica, causada por vírus e pode ser fatal. Muitos são os cães que morrem decorrente da doença.

O presente estudo pretende identificar quais as variáveis que mais contribuem para que o animal, que está contaminado pelo vírus, não resista e venha a morrer, para que se possam minimizar os seus efeitos.

A variável resposta que se tem é binária: o animal morreu ou não morreu. $\mathrm{Na}$ medicina humana é comum utilizarem-se variáveis resposta do tipo binária e o modelo estatístico adequado para tais casos é o modelo de regressão logístico, que além de ser um modelo probabilístico, permite calcular a razão das chances das várias variáveis.

Para a estimação dos parâmetros do modelo, utilizou-se o método da máxima verossimilhança, sendo que o programa estatístico para o cálculo foi o SAS Statistical Analysis System, versão 6.11 para Windows. 
Para exemplificar essa metodologia, foram analisadas 10 (dez) variáveis explanatórias e um total de 641 animais que contraíram a Gastroenterite Hemorrágica e que foram atendidos no Hospital Veterinário da Universidade Estadual de Londrina, no período de 31/08/93 a 31/08/94. Uma análise dos resíduos foi efetuada para se verificar o comportamento dos dados e a existência de pontos influentes.

Observou-se a adequação do modelo ao conjunto de dados, sendo que apenas $1 \%$ dos dados foram considerados discrepantes e cerca de $10 \%$ considerados influentes. Apenas dois pontos, $0.31 \%$, foram considerados discrepantes e influentes ao mesmo tempo. 


\title{
LOGISTIC REGRESSION APPLIED FOR IDENTIFYING RISK FACTORS TO DISEASE IN DOMESTICS ANIMALS
}

\author{
Author: SILVANO CESAR DA COSTA \\ Adviser: Prof. Dr. DÉCIO BARBIN
}

\section{SUMMARY}

In animal medicine, a common disease is Hemorragic Gastroenteritis, which is caused by a virus and may be fatal.

This work aims to identify the variables that most contribute to the death of dogs infected by the virus, so that their effects may be minimized.

The response variable is binary: the animal either died or not. In human medicine, binary response variable are commonly used, and one possible statistical model in such cases is the logistic regression model, which, besides being a probabilistic model, it also allows to calculate the odd ratio of the several variables.

To estimate the model parameters, the maximum likelihood method was used, following the SAS - Statistical Analysis System - Windows, version 6.11 for calculus.

To exemplify the procedure, 10 explanatory variables were analised, and a total of 641 animals infected by Hemorragic Gastroenteritis and assisted at the Veterinary Hospital of Londrina State University were also analised from Aug. 31, 1993 to Aug. 31, 
1994 were also analysed. An analyses of the residuals was carried out to verify data distribution and the existence or not of influencing points.

The adequacy of the model to the data set was observed, and only $1 \%$ of the data was considered to be disagreeing, while about $10 \%$ of the data was influencing. Only two points, $0.31 \%$, were considered to be both disagreeing and influencing. 


\section{INTRODUÇÃO:}

Na prática da pesquisa clínica, é freqüente a necessidade de se estudar a relação entre um fator de risco e uma doença, na presença de um fator de confundimento. A metodologia de Mantel-Haenzel permite resolver este problema se os fatores envolvidos são discretos. Porém, sabe-se que muitas doenças, às vezes, não são causadas por um único agente, mas sim por uma combinação de diferentes circunstâncias, chamadas fatores de exposição ou fatores de risco. No caso do número de fatores de risco e de confundimento presentes no estudo serem maiores que um, a interação entre estes fatores deve ser pesquisada.

Nos aspectos epidemiológicos, os delineamentos básicos usados para a etiologia de uma doença são de coorte e caso-controle. O estudo de coorte envolve o acompanhamento de um grupo de pessoas ao longo de um determinado tempo. No início do estudo, nenhuma das pessoas possui o efeito clínico e são classificadas de acordo com as características (fatores em estudo) que podem estar relacionadas com o efeito clínico. Estas pessoas são acompanhadas ao longo de um tempo, procurando-se o surgimento do efeito clínico. Assim, é possível estabelecer como os fatores iniciais se relacionam com os efeitos subseqüentes. Este estudo possui dois propósitos principais: descritivos, isto é, descrevem a incidência de eventos (efeitos clínicos) no tempo; e analíticos, isto é, permitem a análise das associações entre os fatores em estudo medidos, inicialmente, e os efeitos clínicos medidos ao longo do tempo. São também chamados de estudos de incidência, estudos longitudinais e estudos prospectivos. $\mathrm{O}$ estudo de caso-controle envolve a seleção de duas populações diferentes de pacientes, uma com e outra sem o efeito clínico. A seguir, pesquisa-se a presença ou não do fator em estudo no passado 
(eixo temporal invertido) nas duas populações. Isto facilita a identificação de fatores de risco para doenças raras, com baixo custo. São também chamados de estudos retrospectivos.

O estudo caso-controle foi a primeira ferramenta para o estudo de fatores relacionados à incidência da doença, conforme relata Prentice \& Pike (1979), dado que muitos organismos vivos, vegetais e animais, crescem de acordo com o modelo logístico. Dentro desses estudos a regressão logística é o procedimento mais usado para a análise de dados, quando a variável medida é dicotômica. Este modelo foi sugerido por Berkson (1944), que apresenta o modelo e o método de estimação usando iterativamente mínimos quadrados ponderados, o que tem gerado interesse de vários autores, quanto às suas características matemáticas e quanto ao método de estimação dos parâmetros, devido a sua importância na Biometria para estimar a probabilidade.

Esta técnica é bastante difundida na área médica, porém pouco difundida em outras áreas. Como uma dessas áreas, cita-se a veterinária, especificamente na experimentação com animais. Muitos experimentos e testes estatísticos são realizados em animais produtivos como suínos, aves de corte, gado, etc. Pouca atenção, porém, é dada aos animais que não geram lucros, como por exemplo, cães, que são acometidos por várias doenças, e que são pouco exploradas através de métodos estatísticos, tal como a Gastroenterite Hemorrágica, uma doença que acomete muitos cães na fase jovem, com um grau de mortalidade muito alto e que são atendidos no Hospital Veterinário da Universidade Estadual de Londrina. Os sintomas dessa doença são diarréia com sangue e concomitantemente vômitos, ocasionados por um conjunto de doenças conhecidas e estudadas isoladamente. É uma síndrome que pode ser causada por vírus, vermes, parasitos gastrointestinais e principalmente bactérias.

A literatura descreve fatores importantes que estão relacionados com essas doenças conhecidas, e que dentre eles, podemos citar a idade, raça, porte (peso), estresse ambiental, temperatura ambiental e umidade relativa do ar que favorecem as doenças, porém, ainda consideradas como hipóteses de associação para a Gastroenterite Hemorrágica, devido à falta de um estudo mais conclusivo. 
Através da regressão logística espera-se detectar as variáveis que mais influenciam no óbito de animais com um determinado distúrbio de saúde, calculando a razão das chances dos fatores, sem a preocupação com a doença que o causou. Sendo assim, quando for constatado o sintoma no animal, as variáveis de maior chance de comprometimento do quadro clínico, deverão ser pesquisadas e combatidas mais rapidamente. Com isso espera-se controlar essas variáveis, minimizando o número de animais acometidos por tal doença e que acarretam morte, reduzindo o custo de seu tratamento, e espera-se também disseminar essa técnica a outras áreas de estudo além da área médica.

Como exemplo de aplicação dessa técnica, serão usados dados de Gastroenterite Hemorrágica, de cães. 


\section{REVISÃO DE LITERATURA:}

\section{1. - Modelo Matemático}

A regressão logística tem sido bastante estudada, devido à grande importância na Biometria para o cálculo da probabilidade do desenvolvimento de uma doença, em um período de tempo definido.

Para explicar o grande uso da regressão logística, Kleinbaum (1994) descreve a função logística, apresentando a forma matemática na qual se baseia o modelo logístico:

$$
f(Z)=\frac{1}{1+e^{-Z}},
$$

cujo gráfico é:

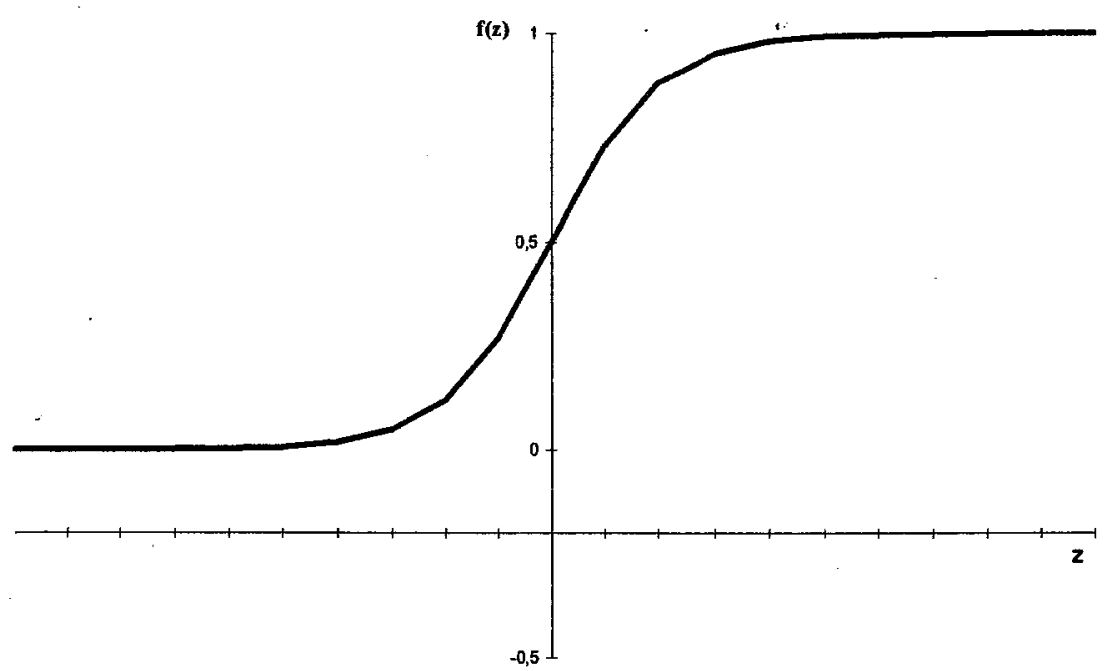


Quando $Z \rightarrow-\infty$, a função tende a zero:

$\lim _{z \rightarrow-\infty} f(Z)=\lim _{Z \rightarrow-\infty} \frac{1}{1+e^{-z}} \Rightarrow f(Z)=0$

e quando $Z \rightarrow \infty \Rightarrow \lim _{Z \rightarrow \infty} f(Z)=\lim _{Z \rightarrow \infty} \frac{1}{1+e^{-z}} \Rightarrow f(Z)=1$

O autor mostra que a função $f(Z)$ assume valores entre 0 e 1 , independente dos valores de $\mathrm{Z}$.

$O$ fato de a função logística $f(Z)$ assumir valores entre 0 e 1 é a primeira razão para o modelo logístico ser tão usado para descrever a probabilidade, que é sempre um número entre 0 e 1 . No contexto epidemiológico, tal probabilidade dá o risco de um indivíduo apresentar um determinado desfecho (doença, morte, recidiva, etc.).

A forma $S$ da função logística agrada aos epidemiologistas que vêem a variável $Z$ como um índice que combina as contribuições de vários fatores de risco, e $f(Z)$ representa o risco para um dado valor de $Z$. Então, a forma $S$ de $f(Z)$ indica que o efeito de $\mathrm{Z}$ sobre um risco individual é mínimo para valores baixos de $Z$, até que algum limiar é alcançado. O risco, então, aumenta rapidamente em certo intervalo de valores $\mathrm{Z}$ intermediários, e então permanece extremamente alto, em torno de 1 , para valores de $Z$ que sejam grandes o suficiente.

Em outras palavras, um modelo com forma $S$ é considerado de grande aplicabilidade por considerar a natureza multivariada de uma questão na pesquisa epidemiológica.

Para obter o modelo logístico de uma função logística, Kleinbaun (1994) escreve $Z$ como: $Z=\beta_{0}+\beta_{1} X_{1}+\beta_{2} X_{2}+\cdots+\beta_{k} X_{k}$, onde $X_{1}, X_{2}, \ldots, X_{k}$ são variáveis independentes de interesse e $\beta_{0}, \beta_{1}, \beta_{2}, \ldots, \beta_{\mathrm{k}}$ são termos constantes representando parâmetros desconhecidos. $\mathrm{Na}$ essência, então, $\mathrm{Z}$ é um indexador que combina os $\mathrm{X}$. Substituindo a soma linear na fórmula para $f(Z)$, resulta:

$$
f(Z)=\frac{1}{1+e^{-\left(\beta_{0}+\beta_{1} X_{1}+\cdot+\beta_{k} X_{k}\right)}}
$$


Para ver esta expressão como um modelo matemático, deve-se colocá-la no contexto epidemiológico. O modelo logístico considera a seguinte estrutura de estudo epidemiológico: tendo observado as variáveis independentes $X_{1}, X_{2}, \ldots, X_{k}$ sobre um grupo de indivíduos, verifica-se o nível de doença (D) e atribui-se o valor 1 ao indivíduo que estiver com a doença e 0 ao indivíduo que estiver sem a doença.

Esta informação é usada para descrever a probabilidade com que a doença se desenvolverá durante um período definido de estudo, digamos $T_{0}$ e $T_{1}$, nos indivíduos livres da doença com os valores das variáveis independentes $X_{1}, X_{2}, \ldots, X_{k}$ que são medidas para $\mathrm{T}_{0}$.

A probabilidade inicialmente modelada pode ser denotada pela probabilidade condicional: $\mathrm{P}\left(\mathrm{D}=1 \mid \mathrm{X}_{1}, \mathrm{X}_{2}, \cdots, \mathrm{X}_{\mathrm{k}}\right)$

$O$ modelo é definido como logístico se a expressão para a probabilidade do desenvolvimento da doença, dadas as variáveis aleatórias independentes $X_{1}, X_{2}, \ldots$, $\mathrm{X}_{\mathrm{k}}$ é dado por:

$$
P\left(D=1 \mid X_{1}, X_{2}, \cdots, X_{k}\right)=\frac{1}{1+e^{-\left(\beta_{0}+\beta_{1} X_{1}+\cdots+\beta_{k} X_{k}\right)}}
$$

Os termos $\beta_{0}, \beta_{1}, \beta_{2}, \ldots, \beta_{\mathrm{k}}$ representam parâmetros desconhecidos, que devem ser estimados, baseado nos valores das variáveis independentes obtidos dos $\mathrm{X}$ 's e do resultado do desfecho $\mathrm{D}$ (resultado da doença) para um grupo de indivíduos.

\section{2. - Estimação de Máxima Verossimilhança}

A estimação dos parâmetros é feita através do método da máxima verossimilhança que não requer restrição de nenhuma espécie sobre as características das variáveis independentes. Deste modo, quando se usa a estimação de máxima verossimilhança, as variáveis independentes podem ser nominais, ordinais ou intervalos.

De acordo com Kleinbaum (1994), a função de verossimilhança L ou L( $\beta$ ), representa a probabilidade conjunta ou verossimilhança dos dados observados que foram 
coletados. Como a função de verossimilhança foi determinada para um certo conjunto de dados, o método da máxima verossimilhança escolherá aquele estimador do conjunto de parâmetros desconhecidos $\beta$, denotado por $\hat{\beta}$, que maximizará a função de verossimilhança $L(\beta)$.

Em geral, maximizar a função de verossimilhança $L(\beta)$ é equivalente a maximizar o logaritmo neperiano ou o logaritmo natural de $L(\beta)$, o que é computacionalmente mais fácil. Os componentes de $\beta$ são encontrados como solução das equações das derivadas parciais, isto é, fazendo-se

$$
\frac{\partial \ln L(\beta)}{\partial \beta_{j}}=0 \quad j=0,1,2, \ldots, k
$$

Se há k parâmetros no total, então o conjunto de equações acima é um conjunto de $\mathrm{k}+1$ equações. Estas equações deverão ser resolvidas iterativamente utilizando-se métodos numéricos. $\mathrm{O}$ método de Newton-Raphson para o $(\mathrm{r}+1)$-ésimo ciclo da interação é dado por:

$$
\hat{\boldsymbol{\beta}}_{\mathrm{r}+1}=\hat{\boldsymbol{\beta}}_{\mathrm{r}}-\mathrm{H}^{-1}\left(\hat{\boldsymbol{\beta}}_{\mathrm{r}}\right) \mathbf{u}\left(\hat{\boldsymbol{\beta}}_{\mathrm{r}}\right), \text { para } \mathrm{r}=0,1,2, \ldots
$$

sendo que, $u\left(\hat{\boldsymbol{\beta}}_{\mathrm{r}}\right)$ é o vetor de escores; H é a matriz Hessiana.

Há, atualmente, duas alternativas de aproximação de máxima verossimilhança que podem ser usadas para estimar os parâmetros no modelo logístico. Estes são chamados de método incondicional e método condicional.

\subsection{1. - Máxima Verossimilhança Incondicional}

Segundo Kleinbaum (1994), a fórmula da verossimilhança incondicional ( $\left.\mathrm{L}_{\mathrm{U}}\right)$ descreve a probabilidade conjunta dos dados como o produto da probabilidade conjunta para os casos (pessoas doentes) e a probabilidade conjunta para não-casos (pessoas não doentes). Estes produtos são usados assumindo-se que as observações são independentes. 


$$
L_{U}=\prod_{l=1}^{m_{l}} P\left(X_{l}\right) \prod_{l=m_{l}+1}^{n}\left[1-P\left(X_{l}\right)\right]
$$

onde: $P(X)=\frac{1}{1+e^{-\left(\beta_{0}+\beta_{1} X_{1}+\cdots+\beta_{k} X_{k}\right)}}$. Substituindo, temos:

$$
L_{U}=\frac{\prod_{l=1}^{n} e^{\left(\beta_{0}+\beta_{1} X_{1}+\cdots+\beta_{k} X_{k}\right)}}{\prod_{l=1}^{n}\left[1+e^{\left(\beta_{0}+\beta_{1} X_{1}+\cdots+\beta_{k} X_{k}\right)}\right]}
$$

A fórmula incondicional tem a vantagem teórica de que ela é desenvolvida diretamente como uma probabilidade conjunta dos dados observados.

\subsection{2. - Máxima Verossimilhança Condicional}

A fórmula da verossimilhança condicional $\left(L_{C}\right)$ dada por Kleinbaum (1994), é:

$$
L_{C}=\frac{\prod_{l=1}^{m_{l}} P\left(X_{l}\right) \prod_{l=m_{l}+1}^{n}\left[1-P\left(X_{l}\right)\right]}{\sum_{u}\left\{\prod_{l=1}^{m_{l}} P\left(X_{u l}\right) \prod_{l=m_{l}+1}^{n}\left[1-P\left(X_{u l}\right)\right]\right\}}
$$

sendo que o numerador é a expressão da verossimilhança indicada para o método incondicional. Quando o autor substitui $\mathrm{P}(\mathrm{X})$, obtém-se:

$$
\mathrm{L}_{\mathrm{c}}=\frac{\prod_{\mathrm{l}=1}^{\mathrm{m}_{\mathrm{l}}} \mathrm{e}^{\sum_{\mathrm{i}=1}^{\mathrm{k}} \beta_{\mathrm{i}} \mathrm{x}_{\mathrm{li}}}}{\sum_{\mathrm{u}}\left[\prod_{\mathrm{l}=1}^{m_{\mathrm{l}}} \mathrm{e}^{\sum_{\mathrm{i}=1}^{\mathrm{k}} \beta_{\mathrm{i}} \mathrm{x}_{\mathrm{lu}_{\mathrm{i}}}}\right]}
$$

$\mathrm{O}$ autor nota que o intercepto foi retirado da verossimilhança e que a remoção do intercepto $\beta_{0}$ da verossimilhança condicional é importante porque significa 
que quando um programa de MV condicional é usado, estimativas são obtidas somente para os coeficientes $\beta_{\mathrm{i}}$ no modelo e não para $\beta_{0}$.

Em particular, se os dados vêm de um estudo de caso-controle, não poderemos estimar $\beta_{0}$, porque não podemos estimar risco, e a função de verossimilhança condicional não permite obter tal estimativa. A fórmula condicional tem a vantagem de possuir um parâmetro a menos para ser estimado que é o intercepto.

$\mathrm{Na}$ escolha entre a aproximação de máxima verossimilhança incondicional e condicional, o pesquisador necessita considerar, primeiramente, o delineamento utilizado e o número de parâmetros no modelo relativo ao número total de indivíduos em estudo. Uma vez definido o melhor delineamento, a estimação de máxima verossimilhança incondicional é preferida se o número de parâmetros no modelo é pequeno em relação ao número de indivíduos. Em contraste, a estimação de máxima verossimilhança condicional é preferida se o número de parâmetros no modelo é grande em relação ao número de indivíduos.

Uma regra segura é usar a estimação de máxima verossimilhança condicional sempre que houver dúvida sobre qual método usar, porque, teoricamente, a aproximação condicional dá sempre resultados não viesados. Em contraste, a aproximação incondicional, quando inadequada, pode dar resultados viesados e, em particular, pode superestimar a razão de chances de interesse.

Assim, após estimar os parâmetros e tendo determinado os valores de $\mathrm{X}_{1}$, $\mathrm{X}_{2}, \ldots, \mathrm{X}_{\mathrm{k}}$ para um particular indivíduo livre da doença, pode-se utilizar a fórmula para obter a probabilidade que este indivíduo tem de desenvolver a doença em algum intervalo de tempo definido.

Por conveniência de notação, denotaremos a probabilidade $P\left(D=1 \mid X_{1}, X_{2}, \cdots, X_{k}\right)$ como simplesmente $\mathbf{P}(\mathbf{X})$ onde o $\mathbf{X}$ é a notação mais simples para a coleção de variáveis $X_{1}, X_{2}, \ldots, X_{k}$. Assim o modelo pode ser escrito como:

$$
P(X)=\frac{1}{1+e^{-\left(\beta_{0}+\beta_{1} X_{1}+\cdots+\beta_{k} X_{k}\right)}} .
$$


Para ilustrar como as inferências estatísticas são feitas usando as estimativas de máxima verossimilhança e seus erros padrões, Kleinbaum (1994) considera os seguintes modelos:

$\log$ it $P_{1}(X)=\beta_{0}+\beta_{1} X_{1}+\beta_{2} X_{2}$

$\log$ it $P_{2}(X)=\beta_{0}+\beta_{1} X_{1}+\beta_{2} X_{2}+\beta_{3} X_{3}$

$\log$ it $P_{3}(X)=\beta_{0}+\beta_{1} X_{1}+\beta_{2} X_{2}+\beta_{3} X_{3}+\beta_{4} X_{1} X_{3}+\beta_{5} X_{2} X_{3}$

e seja $\hat{\mathrm{L}}_{1}, \hat{\mathrm{L}}_{2}$ e $\hat{\mathrm{L}}_{3}$ os valores de verossimilhança maximizados, baseado no ajuste dos modelos, respectivamente. $\mathrm{O}$ ajuste pode ser feito tanto pelo método condicional ou incondicional. A relação entre os $\hat{L}$ 's é similar à propriedade na análise de regressão linear múltipla, onde o modelo com mais parâmetros é o que tem a mais alta estatística $\mathrm{R}^{2}$. Assim, temos que, $\hat{\mathrm{L}}_{1} \leq \hat{\mathrm{L}}_{2} \leq \hat{\mathrm{L}}_{3}$. Segue, de relações algébricas, que:

$\ln \hat{\mathrm{L}}_{1} \leq \ln \hat{\mathrm{L}}_{2} \leq \ln \hat{\mathrm{L}}_{3}$ $-2 \ln \hat{\mathrm{L}}_{3} \leq-2 \ln \hat{\mathrm{L}}_{2} \leq-2 \ln \hat{\mathrm{L}}_{1}$.

A estatística $-2 \ln \hat{\mathrm{L}}_{1}$ é chamada estatística de verossimilhança para o modelo 1, e similarmente, as outras duas estatísticas são as estatísticas de verossimilhança para seus respectivos modelos. Estas estatísticas são importantes porque elas podem ser usadás para testar hipóteses sobre os parâmetros no modelo usando o teste da razão de verossimilhança.

Sabe-se que a diferença entre estatísticas de verossimilhança para dois modelos, um dos quais é um caso especial do outro, tem uma distribuição aproximadamente $\chi^{2}$ em grandes amostras. Tal teste estatístico é chamado razão de verossimilhança ou estatística LR. O número de graus de liberdade (g.l.) para este teste $\chi^{2}$ é igual a diferença entre o número de parâmetros nos dois modelos. 
O modelo com mais parâmetros é chamado modelo completo e o outro é o modelo reduzido; isto é, o modelo reduzido é obtido fazendo certos parâmetros no modelo completo iguais a zero, o que corresponde à hipótese nula inicialmente testada.

Como ilustração, considerem-se os modelos dados anteriormente, e pretende-se comparar o modelo 1 com o modelo 2 :

modelo 2 (modelo completo): $\log$ it $P_{2}(X)=\beta_{0}+\beta_{1} X_{1}+\beta_{2} X_{2}+\beta_{3} X_{3}$

modelo 1 (modelo reduzido): $\log$ it $\mathrm{P}_{1}(\mathrm{X})=\beta_{0}+\beta_{1} \mathrm{X}_{1}+\boldsymbol{\beta}_{2} \mathrm{X}_{2}$

Assim, temos que $\log$ it $P_{2}(X)-\log i P_{1}(X)=\beta_{3} X_{3}$. Considerando $X_{3}$ uma variável dicotômica, fica:

$$
\begin{gathered}
\ln \left(\frac{p_{2}}{1-p_{2}}\right)-\ln \left(\frac{p_{1}}{1-p_{1}}\right)=\beta_{3}(1-0) \\
\ln \left(\frac{\frac{p_{2}}{1-p_{2}}}{\frac{p_{1}}{1-p_{1}}}\right)=\beta_{3}(1-0),
\end{gathered}
$$

sendo que

$$
\frac{\frac{p_{2}}{1-p_{2}}}{\frac{p_{1}}{1-p_{1}}}=\text { OR , }
$$

é chamada razão das chances. Logo, $\ln O R=\beta_{3} \quad \Leftrightarrow \quad O R=e^{\beta_{3}}$.

Assim, a hipótese a ser testada é:

$$
\mathrm{H}_{0}: \beta_{3}=0 \quad \Leftrightarrow \quad \mathrm{H}_{0}: \mathrm{OR}=\mathrm{e}^{0}=1
$$


ou seja, neste caso, testar a hipótese nula de que $\beta_{3}=0$ é equivalente a testar a hipótese nula que a razão de chances ajustada é igual a 1.

Para testar esta hipótese nula, a estatística razão de verossimilhança correspondente é dàda pela diferença: $-2 \log \hat{\mathrm{L}}_{1}-\left(-2 \log \hat{\mathrm{L}}_{2}\right)=-2 \log \left(\frac{\hat{\mathrm{L}}_{1}}{\hat{\mathrm{L}}_{2}}\right)$. Para este exemplo, o número de graus de liberdade é igual a 1, que é a diferença entre o número de parâmetros nos dois modelos.

Outra maneira de fazer inferências sobre os parâmetros é usar o teste de Wald, que é usualmente feito quando há somente um parâmetro inicialmente testado.

O teste estatístico Wald é calculado dividindo-se os coeficientes estimados de interesse por seu erro padrão. Este teste estatístico tem aproximadamente uma distribuição $\mathrm{N}(0,1)$ em grandes amostras. $\mathrm{O}$ quadrado desta estatística é aproximadamente uma estatística $\chi^{2}$ com um grau de liberdade.

Hauck \& Donner (1977) mostram, que para testes de hipóteses relativas a parâmetros simples no modelo "logit" binomial, o teste de Wald tem os seguintes aspectos indesejáveis:

1. para algum tamanho amostral, o valor de $\mathbf{p}$ do teste estatístico de Wald tende a zero quando a distância entre a estimativa do parâmetro e o valor nulo aumentam;

2. o poder do teste de Wald, baseado na sua distribuição de grandes amostras, tende ao nível mínimo de significância para hipóteses alternativas distantes do valor nulo.

Para ilustrar como o teste de Wald pode ser enganoso, os autores usaram os dados coletados por McCormack (1976) em um estudo de mulheres estudantes. Com base em testes de laboratório, o organismo $T$. vaginalis pôde ser isolado em algumas destas mulheres. Uma análise "logit", com 9 variáveis, foi realizada para determinar se a presença de $T$. vaginalis estava relacionado a características específicas das mulheres. A estatística Wald, o teste da razão de verossimilhança e seus valores de probabilidades correspondentes para testar $\mathrm{H}_{0}: \beta_{\mathrm{i}}=0$ vs $\mathrm{H}_{\mathrm{A}}: \beta_{\mathrm{i}} \neq 0, \mathrm{i}=1,2, \ldots 9$, foram calculados $\mathrm{e}$ foi verificado que os valores de probabilidades, para o teste de Wald eram 
consistentemente maiores do que para o teste da razão de verossimilhança. Particularmente, duas variáveis não alcançaram o nível de significância convencional pelo teste de Wald e eram estatisticamente significativas pelo teste da razão de verossimilhança.

Um valor pequeno da estatística diz que o valor atual do parâmetro é próximo ou muito longe do valor nulo? É visível que o teste de Wald tem um comportamento aberrante, falhando em rejeitar quando o coeficiente é significativo. Em face a este problema, os autores recomendam o uso do teste da razão de verossimilhança.

\section{3. - Ajuste do Modelo}

Após estimar os parâmetros sob um conjunto de dados, é natural perguntar se os valores ajustados da variável resposta sobre o modelo retrata bem os valores observados. Se a concordância entre as observações e os correspondentes valores ajustados é boa, o modelo poderá ser aceito. Se não, a forma corrente do modelo certamente não será aceitável e o modelo necessitará ser revisado. Este aspecto de adequação do modelo é referido como qualidade de ajuste do modelo.

Há um número de estatísticas resumo que medem a discrepância entre

proporções binomiais observadas, $\frac{y_{i}}{n_{i}}$, e proporções ajustadas, $\hat{p}_{i}$, sobre um modelo adotado para a verdadeira probabilidade de sucesso $\mathrm{p}_{\mathrm{i}}$. Destes, o mais usado é baseado na função de verossimilhança para o modelo adotado. A função de verossimilhança resume as informações que os dados geram sobre os parâmetros desconhecidos em um dado modelo.

Segundo Collett (1991), o valor da verossimilhança pode ser usado para resumir a extensão para o qual os dados amostrais são ajustados por este modelo corrente, quando os parâmetros desconhecidos são conjuntos iguais às suas estimativas de máxima verossimilhança, Esta é a verossimilhança maximizada sobre o modelo 
corrente, denotada $\hat{\mathrm{L}}_{\mathrm{C}}$. Esta estatística não pode ser usada sobre ela mesma para avaliar a falta de ajuste do modelo corrente, uma vez que ela não é independente do número de observações na amostra.

O autor observa que é necessário, então, comparar o modelo corrente com um modelo básico alternativo para os mesmos dados. Este último modelo é escolhido para ser o modelo para o qual os valores ajustados coincidem com as observações atuais, isto é, um modelo que ajusta os dados perfeitamente. Tal modelo terá o mesmo número de parâmetros desconhecidos quanto há observações. $\mathrm{O}$ modelo é chamado completo ou saturado e a verossimilhança maximizada sobre ele é denotada por $\hat{\mathrm{L}}_{\mathrm{f}}$. O modelo completo não é útil por si só, uma vez que ele não gera um resumo mais simples dos dados do que as próprias observações individuais. Contudo, pela comparação de $\hat{\mathrm{L}}_{\mathrm{c}}$ com $\hat{\mathrm{L}}_{\mathrm{f}}$, o grau para o qual o modelo corrente represente adequadamente os dados pode ser avaliado.

Para comparar $\hat{\mathrm{L}}_{\mathrm{C}}$ e $\hat{\mathrm{L}}_{\mathrm{f}}$, é conveniente usar menos duas vezes o logaritmo da razão destas verossimilhanças maximizadas, o qual é denotado por $\mathrm{D}$, ou

$$
D=-2 \log \left(\frac{\hat{\mathrm{L}}_{\mathrm{C}}}{\hat{\mathrm{L}}_{\mathrm{f}}}\right)=-2\left[\log \hat{\mathrm{L}}_{\mathrm{C}}-\log \hat{\mathrm{L}}_{\mathrm{f}}\right] .
$$

Grandes valores de $D$ são encontrados quando $\hat{L}_{c}$ é pequeno em relação a $\hat{L}_{f}$, indicando que o modelo corrente é um modelo pobre. Por outro lado, valores pequenos de $\mathrm{D}$ são obtidos quando $\hat{\mathrm{L}}_{\mathrm{c}}$ é similar a $\hat{\mathrm{L}}_{\mathrm{f}}$, indicando que o modelo corrente é um bom modelo. A estatística $\mathrm{D}$, mede então, o grau para o qual o modelo corrente se afasta do modelo completo e é chamada "deviance".

$\mathrm{Na}$ modelagem de $\mathrm{n}$ observações binomiais, onde $\mathrm{p}_{i}$ é a verdadeira probabilidade de sucesso correspondendo à i-ésima observação $\frac{y_{i}}{n_{i}}, i=1,2, \ldots, n, a$ função de verossimilhança é 


$$
\prod_{i=1}^{n}\left(\begin{array}{l}
n_{i} \\
y_{i}
\end{array}\right) p_{i}^{y_{i}}\left(1-p_{i}\right)^{n_{i}-y_{i}}
$$

Ao ajustar um modelo logístico linear com $(k+1)$ parâmetros desconhecidos $\beta_{0}, \beta_{1}, \cdots, \beta_{k}$, os valores ajustados $\hat{p}_{i}$ são obtidos, onde

$$
\operatorname{logit}\left(\hat{\mathrm{p}}_{\mathrm{i}}\right)=\hat{\beta}_{0}+\hat{\beta}_{1} \mathrm{x}_{1 \mathrm{i}}+\hat{\beta}_{2} \mathrm{x}_{2 \mathrm{i}}+\cdots+\hat{\beta}_{\mathrm{k}} \mathrm{x}_{\mathrm{ki}} .
$$

O logaritmo da função de verossimilhança maximizado para o modelo corrente é dado por:

$$
\log \hat{L}_{c}=\sum_{i}\left\{\log \left(\begin{array}{l}
n_{i} \\
y_{i}
\end{array}\right)+y_{i} \log \hat{p}_{i}+\left(n_{i}-y_{i}\right) \log \left(1-\hat{p}_{i}\right)\right\} .
$$

Para o modelo completo, as probabilidades ajustadas serão as mesmas que as proporções observadas $\widetilde{p}_{i}=\frac{y_{i}}{n_{i}}, i=1,2, \ldots, n, e$ também, o logaritmo da função de verossimilhança para o modelo completo é:

$$
\log \hat{\mathrm{L}}_{\mathrm{f}}=\sum_{\mathrm{i}}\left\{\log \left(\begin{array}{l}
\mathrm{n}_{\mathrm{i}} \\
\mathrm{y}_{\mathrm{i}}
\end{array}\right)+\mathrm{y}_{\mathrm{i}} \log \widetilde{\mathrm{p}}_{\mathrm{i}}+\left(\mathrm{n}_{\mathrm{i}}-\mathrm{y}_{\mathrm{i}}\right) \log \left(1-\widetilde{\mathrm{p}}_{\mathrm{i}}\right)\right\} .
$$

A "deviance" é, então, dada por:

$$
\begin{gathered}
D=-2\left[\log \hat{\mathrm{L}}_{\mathrm{c}}-\log \hat{\mathrm{L}}_{\mathrm{f}}\right] \\
\mathrm{D}=2 \sum_{\mathrm{i}}\left\{\mathrm{y}_{\mathrm{i}} \log \left(\frac{\widetilde{\mathrm{p}}_{\mathrm{i}}}{\hat{\mathrm{p}}_{\mathrm{i}}}\right)+\left(\mathrm{n}_{\mathrm{i}}-\mathrm{y}_{\mathrm{i}}\right) \log \left(\frac{1-\widetilde{\mathrm{p}}_{\mathrm{i}}}{1-\hat{\mathrm{p}}_{\mathrm{i}}}\right)\right\} .
\end{gathered}
$$

Se o número ajustado de sucessos sobre o modelo corrente é $\hat{y}_{i}=n_{i} \hat{p}_{i}$, a "deviance" pode ser escrita por:

$$
D=2 \sum_{i}\left\{y_{i} \log \left(\frac{y_{i}}{\hat{y}_{i}}\right)+\left(n_{i}-y_{i}\right) \log \left(\frac{n_{i}-y_{i}}{n_{i}-\hat{y}_{i}}\right)\right\}
$$

e é facilmente visto que esta é uma estatística que compara as observações $y_{i}$ com seus correspondentes valores ajustados $\hat{y}_{i}$ sobre o modelo corrente.

No importante caso especial de dados binários, onde $n_{i}=1$ para $\mathrm{i}=1,2, \cdots, \mathrm{n}, \mathrm{a}$ "deviance" depende somente das probabilidades de sucesso ajustadas $\mathrm{p}_{\mathrm{i}}$, 
e também não é informativa sobre a qualidade do ajuste de um modelo. Para ver isto, a verossimilhança para $n$ observações binárias, como uma função dos parâmetros $\beta$, é $L(\beta)=\prod_{i} p_{i}^{y_{i}}\left(1-p_{i}\right)^{1-y_{i}}$ e a função de verossimilhança maximizada sobre algum modelo corrente é:

$$
\log \hat{\mathrm{L}}_{\mathrm{c}}=\sum_{\mathrm{i}}\left\{\mathrm{y}_{\mathrm{i}} \log \hat{\mathrm{p}}_{\mathrm{i}}+\left(1-\mathrm{y}_{\mathrm{i}}\right) \log \left(1-\hat{\mathrm{p}}_{\mathrm{i}}\right)\right\}
$$

Para o modelo completo, $\hat{p}_{i}=y_{i}$, e uma vez que $y_{i} \log y_{i}$ e $\left(1-y_{i}\right) \log \left(1-y_{i}\right)$ são ambos zero para somente dois valores possíveis de $y_{i}, 0$ e 1 , $\log \hat{L}_{f}=0$. A “deviance" para dados binários resulta então:

$$
\begin{aligned}
& D=-2 \sum_{i}\left\{y_{i} \log \hat{p}_{i}+\left(1-y_{i}\right) \log \left(1-\hat{p}_{i}\right)\right\} \\
& D=-2 \sum_{i}\left\{y_{i} \log \left(\frac{\hat{p}_{i}}{1-\hat{p}_{i}}\right)+\log \left(1-\hat{p}_{i}\right)\right\}
\end{aligned}
$$

Agora, a diferenciação de $\log L(\beta)$ com relação ao j-ésimo parâmetro, $\beta_{\mathrm{j}}$, gera

$$
\frac{\partial \log L(\beta)}{\partial \beta_{j}}=\sum_{i}\left\{\frac{y_{i}}{p_{i}}-\frac{1-y_{i}}{1-p_{i}}\right\} p_{i}\left(1-p_{i}\right) x_{j i}
$$

do qual

$$
\sum_{i} \beta_{j} \frac{\partial \log L(\beta)}{\partial \beta_{j}}=\sum_{i}\left(y_{i}-p_{i}\right) \sum_{j} \beta_{j} x_{j i}=\sum_{i}\left(y_{i}-p_{i}\right) \log \frac{p_{i}}{1-p_{i}}
$$

Porque $\hat{\boldsymbol{\beta}}$ é a estimativa de máxima verossimilhança de $\boldsymbol{\beta}$, a derivada do lado esquerdo desta equação é zero para $\hat{\boldsymbol{\beta}}$. Conseqüentemente, as probabilidades ajustadas $\hat{\mathrm{p}}_{\mathbf{i}}$ devem satisfazer a equação:

$$
\sum_{i}\left(y_{i}-\hat{p}_{i}\right) \log \text { it }\left(\hat{p}_{i}\right)=0
$$


e também

$$
\sum_{\mathbf{i}} \mathrm{y}_{\mathrm{i}} \operatorname{logit}\left(\hat{\mathrm{p}}_{\mathbf{i}}\right)=\sum_{\mathbf{i}} \hat{\mathrm{p}}_{\mathrm{i}} \log \mathrm{it}\left(\hat{\mathrm{p}}_{\mathrm{i}}\right)
$$

Finalmente, substituindo por $\sum_{i} y_{i} \log i t\left(\hat{p}_{i}\right)$ na equação 1 , a "deviance" torna-se: $D=-2 \sum_{i}\left\{\hat{p}_{i} \log i t\left(\hat{p}_{i}\right)+\log i t\left(1-\hat{p}_{i}\right)\right\}$

Esta "deviance" depende das observações binárias $y_{i}$ somente através das probabilidades ajustadas $\hat{p}_{i}$ e também não pode nos dizer algo sobre a concordância entre as observações e suas correspondentes probabilidades ajustadas. Conseqüentemente, a "deviance" ao ajustar um modelo para dados de resposta binária não pode ser usada como uma medida resumo da qualidade do ajuste do modelo.

Copenhaver \& Mielke (1977) apresentam uma nova distribuição de tolerância e procedimento de inferência para dados binários. Este método, chamado análise "quantit", é capaz de ajustar uma grande variedade de formas de curvas de respostas. Casos específicos deste novo método incluem análise binária baseada na distribuição exponencial dupla, a distribuição logística (análise "logit"), e a distribuição uniforme. Descrevem, também, técnicas computacionais usadas para implementar o procedimento de verossimilhança associado a este método.

A análise "quantit" é baseada sobre uma distribuição de probabilidade associada a propriedades de uma classe de testes de ordem para duas-amostras. Os autores designam esta distribuição de probabilidade por distribuição ômega e sua função de distribuição acumulada e função densidade $\mathrm{f}(\mathrm{x})$ são caracterizadas por:

$$
\begin{aligned}
& F[x(q)]=q \\
& f[x(q)]=1-|2 q-1|^{v+1}
\end{aligned}
$$




$$
x(q)=\int_{1 / 2}^{q} \frac{d z}{f[x(z)]}
$$

Aqui $F(x)$ não é especificado diretamente, mas

$$
F^{-1}(q)=\int_{1 / 2}^{q} \frac{d z}{\left(1-|2 z-1|^{v+1}\right)} \text { para } 0 \leq q \leq 1
$$

onde $v>-1$.

Em particular, esta distribuição é a exponencial dupla quando $v=0$; uma distribuição logistica quando $v=1$; e uma distribuição uniforme nos casos limites com $v \rightarrow \infty$.

\subsubsection{Distribuição Logística $(v=1)$ :}

$$
\begin{gathered}
f[x(q)]=1-|2 q-1|^{2}=4 q(1-q) \\
x(q)=\int_{1 / 2}^{q} \frac{d z}{4 z(1-z)}=\frac{1}{4} \ln \left(\frac{q}{1-q}\right)
\end{gathered}
$$

que é o "logit" de q.

$$
\text { Assim, } q=F[x(q)]=\frac{e^{4 x}}{1+e^{4 x}} \quad \text { ou } \quad F(x)=\frac{1}{\left(1+e^{-4 x}\right)}, \quad-\infty \leq x \leq \infty
$$

Como $f[x(q)]=4 q(1-q)$, substituindo o valor de $q$, temos a função densidade logística, dada por

$$
f(x)=\frac{4 e^{4 x}}{\left(1+e^{4 x}\right)^{2}} .
$$

Esta função também pode ser obtida derivando-se $\mathrm{F}(\mathrm{x})$. 
2.3.2. Distribuição Exponencial Dupla $(v=0)$ :

$$
\begin{aligned}
& f[x(q)]=1-|2 q-1| \\
& x(q)=\int_{1 / 2}^{q} \frac{d z}{1-|2 z-1|}
\end{aligned}
$$

Similarmente, a função densidade exponencial dupla é dada por

$$
\begin{gathered}
f(x)=e^{-2|x|} \\
f(x)=F^{\prime}(x)=e^{-2|x|}, \quad-\infty \leq x \leq \infty
\end{gathered}
$$

2.3.3. Distribuição Uniforme $(v \rightarrow \infty)$ :

$$
\begin{aligned}
& f[x(q)]=\lim _{v \rightarrow \infty}\left[1-|2 q-1|^{v+1}\right] \\
& x(q)=\lim _{v \rightarrow \infty}\left[\int_{1 / 2}^{q} \frac{d z}{1-|2 z-1|^{v}}\right]
\end{aligned}
$$

A função densidade uniforme é dada por:

$$
\mathrm{f}(\mathrm{x})= \begin{cases}1, & \text { para }-0,5<\mathrm{x}<0,5 \\ 0, & \text { caso }- \text { contrario, quando } v \rightarrow \infty\end{cases}
$$

O modelo "quantit", portanto, cobre uma grande extensão de formas possíveis para $\mathrm{F}(\mathrm{x})$, e permite um teste simples de razão de verossimilhança de $v=1$. Ele é ajustado usando o método da máxima verossimilhança, e aninhando a maximização em relação a $v$, isto é, $v$ é fixado. Estimativas de máxima verossimilhança de $\alpha$ e $\beta$ são obtidas pelo método escore; $v$ é mudado, a maximização é repetida e assim por diante. Finalmente, seleciona-se o $v$ que geram a máxima verossimilhança total. 
A análise "quantit" foi aplicada em 22 conjuntos de dados (enumerados de 1 a 22). Em cinco desses conjuntos o modelo "quantit" produziu um melhor ajuste significativo (ao nível de 5\%) para os dados, que o modelo "logit". Estas comparações sugerem que estimativas de intervalos de dosagens extremas (por ex., ED95 e ED99) baseados na análise "logit" e "probit", para a maior parte, ou são excessivamente otimistas ou excessivamente pessimistas.

Prentice \& Pike (1979) identificaram condições robustas para as quais o modelo de regressão logística pudesse ser usado em dados de caso-controle. Estes resultados dão uma flexibilidade e um método de análise conveniente para estudos de caso-controle nos quais os tamanhos dos estratos sejam razoavelmente grandes. É mostrado que os estimadores da razão das chances e suas matrizes de variâncias e covariâncias assintóticas podem ser obtidas pela aplicação do modelo de regressão logística ao estudo de caso-controle, como se os dados tivessem sido obtidos em um estudo de coorte.

O trabalho estende os resultados de Anderson (1972) sobre diferença logística e generaliza a descoberta de Breslow \& Powers (1979) sobre a equivalência dos estimadores da razão das chances, quando ambos modelos logísticos, prospectivo e retrospectivo, são aplicados a dados de caso-controle.

Aranda-Ordaz (1981) mostra que para analisar a dependência de dados de resposta binária sobre variáveis explanatórias é comum ajustar transformações de probabilidades pela função linear dos parâmetros. $\mathrm{O}$ autor acredita que a transformação logística é a mais usada, porém, faz considerações a respeito da adequacidade do modelo. $\mathrm{O}$ autor considera importante tentar descobrir algum modelo não-logistico e que dê um ajuste mais simples ou melhor. Considera que se for possível ajustar um procedimento que detecte inadequacidade, e que também indique o tipo de modificação desejável para o modelo, a utilidade disto será muito grande.

Um aparente e informativo caminho encontrado pelo autor, para esse objetivo, foi construir modelos extendidos que incluiam o logístico e os alternativos de interesse, como casos especiais. Todos os modelos considerados no artigo têm uma 
transformação associada; isto é, a aproximação considerada consiste na definição de famílias de transformação de probabilidade implícita.

Foram consideradas, primeiro, transformações que são simétricas, que conduzem essencialmente para a mesma resposta de sucessos e falhas que estão alternados. A transformação linear é usada para definir uma família de modelos alternativos para a logística. No caso assimétrico o complemento log-log é empregado como um modelo básico.

Em geral, são comuns situações envolvendo alguns conjuntos de dados heterogêneos. $O$ objetivo do autor foi encontrar um resumo simples da variação entre conjuntos por meio de modelos aditivos para as probabilidades, sobre uma escala determinada pela transformação adequada. Concluiu que uma escolha final do modelo deve depender parcialmente da facilidade com que as conclusões podem ser apresentadas e entendidas.

Motivado pela análise "quantit" proposta por Copenhaver e Mielke (1977), Morgan (1985) apresenta modelos com três e quatro parâmetros que foram propostos para dados binários. $\mathrm{O}$ autor acredita que estes modelos são úteis para decidir se o ajuste de modelos padrões mais simples, tal como o "logit", podem ser melhorados; melhores ajustes resultam em melhores determinações dos níveis de dose extrema. Entretanto, uma desvantagem, citada pelo autor, destes novos modelos é que eles são, muitas vezes, dificeis de se ajustarem aos dados, e também são improváveis de serem muito usados. Um destes modelos é bem-aproximado por um modelo muito mais simples, para uma grande variedade de casos, e estimativas de máxima verossimilhança dos parâmetros para este modelo podem ser obtidas pelo método escore.

Assim, o autor considera os seguintes dois modelos para dados binários. Se $P_{i}=\bar{F}\left(x_{i}\right), \quad 1 \leq i \leq k$, modelo A: $\quad \bar{F}(x)=\frac{1}{1+e^{-\left(\alpha+\beta x+x^{2}+\delta x^{3}\right)}}, \mathrm{e}$ modelo B: $\quad \bar{F}(x)=\frac{1}{1+e^{-\left[\beta(x-\mu)+\gamma(x-\mu)^{3}\right]}}, \quad-\infty \leq x \leq \infty$, ou 


$$
\mathrm{P}(\mathrm{x})=\mathrm{F}(\mathrm{x})=\frac{1}{1+\mathrm{e}^{-\left[\beta(\mathrm{x}-\theta)+\gamma(\mathrm{x}-\theta)^{3}\right]}}
$$

Da comparação dos ajustes dos modelos A e B para os 22 conjuntos de dados de Copenhaver \& Mielke, o autor encontrou que a melhora do ajuste obtido quando se usou o modelo A foi somente marginal, com o custo de um parâmetro adicional. Portanto, concluiu que se deve considerar somente o modelo B. Para este modelo, o algoritmo do método escore segue linha padrão. Assim como o modelo "quantit", o modelo B contém o modelo "logit", quando $\gamma=0$.

Se $\gamma<0, \overline{\mathrm{F}}(\mathrm{x})$ não é uma função monotônica de $\mathrm{x}$, embora ela possa ser monotônica crescente para o intervalo dos $\mathrm{x}$ valores gerados. Para o conjunto de dados analisados, somente um conjunto de dados, foi ascendente para $\hat{\gamma}<0$, e neste caso, o teste estatístico escore não gera evidência de que $\gamma \neq 0$. Quando $\gamma<0$, níveis de dose extrema, tal como $\mathrm{ED}_{99}$, não existem, ou não são únicas. Para o conjunto de dados $4 \mathrm{a}$ $\mathrm{ED}_{99}$ não existe, mas neste caso o intervalo das doses consideradas não incluem a $\mathrm{ED}_{99}$ estimada de outros modelos. $O$ autor deveria discutir depois o caso de $\gamma<0$, mas como ele não foi considerado útil na prática, as análises se concentraram no caso $\gamma \geq 0$.

Dos 22 conjuntos de dados usados por Copenhaver \& Mielke, Morgan utiliza quatro casos daquele conjunto de cinco onde a análise "quantit" produziu um melhor ajuste que o modelo "logit" (sendo que o quinto conjunto de dados rejeitado, foi excluído porque a resposta $0 \%$ ou $100 \%$ foi obtida para 8 dos 12 níveis de dose), e também, para comparação, quatro dos 17 casos remanescentes, escolhidos para medir um amplo intervalo de valores $\hat{v}$. Dos testes de razão de verossimilhança, o modelo $B$ gerou um melhor ajuste significativo do que o modelo "logit" para os mesmos quatro conjuntos de dados, como no modelo "quantit". É interessante que somente no caso do conjunto de dados 4 foi impossível ajustar o modelo $\mathrm{B}$ com $\gamma>0$, e dos oito conjuntos de dados considerados, este é $o$ único onde $\hat{v}<1$.

$\mathrm{O}$ autor usou estes conjuntos de dados somente como meio de comparação de desempenho dos três modelos. Do ponto de vista prático, uma discussão 
do $\mathrm{ED}_{99}$ no contexto do conjunto de dados publicados é irreal a menos que os experimentos tenham sido planejados com o propósito de estimar um nível de dose extrema. O ruim da extrapolação, mencionado por Finney (1971), é o fato de a estimativa ED99 se encontrar fora do intervalo de doses empregadas, e estar presente em cinco dos oito conjuntos de dados considerados acima,

A limitada pesquisa empírica do artigo sugere que o modelo B é uma alternativa útil e simples, para o modelo de análise "quantit", no caso onde $v \geq 1$. Além disso o mesmo não tem o problema de possível convergência de análise "quantit" quando $v$ é grande. Somente para o conjunto de dados 18 tira-se uma conclusão diferente ao usar o teste escore, que é melhor que o teste da razão de verossimilhança, ao nivel de significância de 5\%. O modelo B pode ser ajustado pelo GLIM, encaixando a otimização em relação a. $\mu$. Como $\mu=\mathrm{ED}_{50}$, é possível que este modelo seja bem estimado pelo ajuste do modelo "logit".

Sendo assim, o autor acredita ser possível que o modelo cúbico com $\gamma<0$ possa ser uma alternativa útil para o modelo "quantit" quando $v<1$. O problema de estimativas não-únicas de nível de dose extremas pode ser prontamente resolvido para qualquer conjunto particular de dados. Entretanto, o assunto merece ser melhor estudado.

A flexibilidade da distribuição logística cúbica pode também ser útil em outros modelos que fazem suposições distribucionais específicas, de acordo com a visão do autor. É útil ter uma distribuição simples, de uma variável aleatória ilimitada, que pode gerar uma boa aproximação para a distribuição uniforme. Para o modelo "quantit" uma aproximação ocorre para $v$ grande. Isto é, para o conjunto de dados $14, \hat{v}=13,06$, e aqui o ajuste logístico-cúbico é similar ao resultado obtido pelo uso da distribuição uniforme adjacente. Para o conjunto de dados $11, \hat{v}=9,94$, mas o ajuste logístico-cúbico é agora totalmente diferente. Isto não dificulta imaginar situações nas quais as curvas dose-resposta logístico-cúbico das formas ajustadas para os conjuntos de dados 4 e 11 possam ser apropriados. 
Soares \& Colosimo (1995) definem a regressão logística como uma generalização da técnica de Mantel-Haenszel e exemplificam com um estudo realizado por Brown da Universidade de Stanford, com câncer de próstata. O objetivo do trabalho foi medir a capacidade de predição para o envolvimento nodal de 5 variáveis préoperatórias, cuja coleta é menos invasiva que uma cirurgia. As variáveis consideradas foram: idade do paciente à época do diagnóstico (em anos); nível de fosfatase ácida ( $\mathrm{x}$ 100); resultado da radiografia; resultado da biópsia e medida do estadio do tumor (grau).

O modelo ajustado foi:

$$
\log \left(\frac{p_{i}}{1-p_{i}}\right)=\beta_{0}+\beta_{1} X_{1}+\beta_{2} X_{2}+\cdots+\beta_{5} X_{5}
$$

$\mathrm{i}=1,2, \ldots, \mathrm{n}$. Pelos resultados apresentados, verificou-se que as variáveis idade e grau, não tiveram poder preditivo significativo, sendo que as outras variáveis foram significativas.

Dada a grande aplicabilidade e uso da regressão logistica na área médica, Franco (1988) desenvolveu um programa computacional em linguagem Pascal (Turbo Pascal, versão 4.0, Borland Internacional), com apoio da organização Mundial da Saúde, para regressão logística, que nos dá os parâmetros estimados, erro padrão, risco relativo e seus intervalos de confiança, estimativas do logaritmo da função de verossimilhança antes e depois da convergência e os testes escore e razão de verossimilhança para qualidade do ajustamento.

\section{4. - Razão das Chances}

Collett (1991) fez comparações entre os modelos "logit", "probit" e complemento $\log -\log$ e concluiu que a transformação logística é preferivel em relação às outras, devido a ter uma interpretação direta em termos de logaritmo da chance em favor do sucesso e porque o modelo baseado na transformação logística é, particularmente, apropriado para a análise de dados que tenham sido coletados retrospectivamente, tais como no estudo de caso-controle. 
$\mathrm{O}$ autor alerta para as variáveis de confundimento, que podem mascarar resultados de fatores de interesse. Exemplifica com o estudo de Framingham, cujo objetivo foi investigar, se doenças coronárias estavam associadas ao alto consumo de álcool. Concluiu, que a razão das chances de doenças coronárias em pessoas que consomem grande quantidade de álcool, em relação àquelas de consumo baixo, é 4,64. Feita uma estratificação dos indivíduos, quer eles fumassem ou não, verificou-se que o consumo de álcool não está relacionado com a doença. Assim, o hábito de fumar é uma variável de confundimento.

$\mathrm{O}$ autor indica que há vários caminhos no qual um modelo ajustado possa ser inadequado. O mais importante deles, segundo o autor, é aquele em que o componente sistemático linear do modelo possa ser incorretamente especificado e cita, como exemplo, um modelo que possa não incluir variáveis explanatórias que realmente deveriam estar no modelo, ou ainda, valores considerados por uma ou mais das variáveis explanatórias e que necessitem de transformação. A transformação da probabilidade de resposta usada pode não ser correta; pode ser que uma transformação logística de uma probabilidade de resposta binária tenha sido usada quando teria sido mais apropriado usar a transformação complemento log-log. Os dados podem conter observações particulares, chamadas "outliers", que não são bem ajustadas pelo modelo, ou observações, chamadas pontos influentes, que têm um impacto inadequado sobre as conclusões a serem tiradas das análises. Finalmente, a suposição de que os dados da resposta observada vem de uma distribuição de probabilidade particular, por exemplo, uma distribuição binomial, pode não ser válida.

O autor também diz que algumas vezes é útil descrever a chance que uma variável resposta binária leva ao sucesso em termos de chance daquele evento. A chance de um sucesso é definida como sendo a razão da probabilidade de um sucesso para a probabilidade de uma falha. Isto é, se $\mathrm{p}$ é a verdadeira probabilidade de sucesso, a chance de um sucesso é $\frac{p}{1-p}$. Se os dados binários observados consistem de y sucessos em $n$ 
observações, a chance de um sucesso pode ser estimado por $\frac{\hat{p}}{1-\hat{p}}=\frac{y}{n-y}$. Quando dois conjuntos de dados binários são comparados, uma medida relativa das chances de um sucesso em um conjunto relativo àquele outro é a razão de chances. Suponha que $p_{1}$ e $p_{2}$ são as probabilidades de sucesso nestes dois conjuntos; também que a chance de um sucesso no i-ésimo conjunto é $\frac{p_{i}}{1-p_{i}}, i=1,2$. A razão de chances de um sucesso em um conjunto de dados binários relativo a outro é usualmente denotada por

$$
\psi=\frac{p_{1} /\left(1-p_{1}\right)}{p_{2} /\left(1-p_{2}\right)} .
$$

Quando as chances de um sucesso em cada um dos dois conjuntos de dados binários são idênticas, $\psi$ é igual a 1 . Isto acontecerá quando as duas probabilidades de sucesso forem iguais. Valores de $\psi$ menores do que 1 sugerem que as chances de um sucesso são menores no primeiro conjunto de dados do que no segundo, enquanto uma razão de chances maior do que 1 indica que a chance de um sucesso é maior no primeiro conjunto de dados. A razão das chances é uma medida da diferença entre duas probabilidades de sucesso que pode levar a algum valor positivo, contrário à diferença entre duas probabilidades de sucesso $p_{1}-p_{2}$, que é restrito à amplitude $(-1,1)$.

Hosmer \& Lemeshow (1989) usam a razão das chances para dar uma interpretação dos coeficientes de regressão logística na situação onde a variável independente é dicotômica.

Os autores assumem que a variável $\mathrm{X}$ tem dois valores possíveis, 0 e 1 , onde se têm dois valores de $\mathrm{p}(\mathrm{x})$ e, equivalentemente, dois valores de $1-\mathrm{p}(\mathrm{x})$. Colocando estes valores em uma tabela de contingência $2 \times 2$, considerando o modelo

$$
\mathrm{P}(\mathrm{x})=\frac{\mathrm{e}^{\beta_{0}+\beta_{\mathrm{X}} \mathrm{X}}}{1+\mathrm{e}^{\beta_{0}+\beta_{\mathrm{J}} \mathrm{X}}},
$$


tem-se:

Tabela 1: Presença ou Ausência da Variável Independente em Relação à Variável Dependente no Modelo Logístico

\begin{tabular}{c|cc}
\hline \multirow{2}{*}{$\begin{array}{c}\text { Variável } \\
\text { Dependente }\end{array}$} & $\mathrm{x}=1$ & $\mathrm{x}=0$ \\
\cline { 2 - 3 } $\mathrm{y}=1$ & $P(X=1)=\frac{e^{\beta_{0}+\beta_{1}}}{1+e^{\beta_{0}+\beta_{1}}}$ & $P(X=0)=\frac{e^{\beta_{0}}}{1+e^{\beta_{0}}}$ \\
$\mathrm{y}=0$ & $1-P(X=1)=\frac{1}{1+e^{\beta_{0}+\beta_{1}}}$ & $1-P(X=0)=\frac{1}{1+e^{\beta_{0}}}$ \\
\hline
\end{tabular}

A razão das chances, denotada por $\psi$, é definida como

$$
\begin{gathered}
\psi=\frac{\frac{\mathrm{p}_{1}}{1-\mathrm{p}_{1}}}{\frac{\mathrm{p}_{0}}{1-\mathrm{p}_{0}}}=\frac{\mathrm{p}_{1}\left(1-\mathrm{p}_{0}\right)}{\left(1-\mathrm{p}_{1}\right) \mathrm{p}_{0}} \\
\psi=\frac{\left[e^{\beta_{0}+\beta_{1}} /\left(1+e^{\beta_{0}+\beta_{1}}\right)\right]\left[1 /\left(1+e^{\beta_{0}}\right)\right]}{\left[1 /\left(1+e^{\beta_{0}+\beta_{1}}\right)\right]\left[e^{\beta_{0}} /\left(1+e^{\beta_{0}}\right)\right]} \\
\psi=\frac{\mathrm{e}^{\beta_{0}+\beta_{1}}}{\mathrm{e}^{\beta_{0}}}=\mathrm{e}^{\beta_{1}}
\end{gathered}
$$

e o logaritmo da razão das chances é dado por: $\ln (\psi)=\ln \left(\mathrm{e}^{\beta_{1}}\right)=\beta_{1}$

$\mathrm{O}$ fato de o próprio parâmetro ser interpretado como a razão das chances é a principal razão de a regressão logística ser a ferramenta mais poderosa na pesquisa epidemiológica.

Se Y denota a presença ou ausência de uma determinada doença (câncer de pulmão) e se $\mathrm{X}$ denota, quer ou não, a pessoa possua um atributo (fumante ou não), 
então $\hat{\psi}=2$, por exemplo, indica que câncer de pulmão é 2 vezes mais provável de ocorrer em fumantes do que em não fumantes.

Uma outra forma de estimar a razão das chances de um sucesso em um conjunto de dados relativo a outro é dado por Collett (1991), da seguinte forma:

Suponha que os dados binários sejam organizados, como na seguinte tabela de contingência $2 \times 2$ :

Tabela 2: Freqüências Observadas do Número de Sucessos e Falhas em Relação aos Conjuntos de Dados 1 e 2

\begin{tabular}{c|c|c}
\hline & Número de Sucessos & Número de Falhas \\
\hline Conjunto de Dados 1 & $\mathrm{a}$ & $\mathrm{b}$ \\
Conjunto de Dados 2 & $\mathrm{c}$ & $\mathrm{d}$ \\
\hline
\end{tabular}

As probabilidades de sucesso estimadas nos dois conjuntos de dados são $\hat{p}_{1}=\frac{a}{a+b}$ e $\hat{\mathrm{p}}_{2}=\frac{\mathrm{c}}{\mathrm{c}+\mathrm{d}}$, e também a razão de chances estimadas, $\hat{\psi}$, é dada por:

$$
\hat{\psi}=\frac{\hat{p}_{1} /\left(1-\hat{p}_{1}\right)}{\hat{p}_{2} /\left(1-\hat{p}_{2}\right)}=\frac{a d}{b c}
$$

Esta estimativa é a razão do produto de dois pares dos elementos diagonais na tabela 2 x 2 acima, e por esta razão é algumas vezes referida como a razão do produto cruzado.

A fim de construir um intervalo de confiança para a verdadeira razão das chances, usa-se o logaritmo da razão das chances estimado que é melhor aproximado por uma distribuição normal do que a própria razão das chances, especialmente quando o número total de observações binárias não é muito grande. $\mathrm{O}$ erro padrão aproximado do logaritmo da razão das chances estimado, $\log \hat{\psi}$, é dado por:

$$
\text { s.e. }(\log \hat{\psi}) \approx \sqrt{\frac{1}{\mathrm{a}}+\frac{1}{\mathrm{~b}}+\frac{1}{\mathrm{c}}+\frac{1}{\mathrm{~d}}} \text {. }
$$


Um intervalo de confiança aproximado a $100(1-\alpha) \%$ para log $\psi$ varia de $\left[\log \hat{\psi}-z_{\alpha / 2}\right.$ s.e. $(\log \hat{\psi}) ; \log \hat{\psi}+z_{\alpha / 2}$ s.e. $\left.(\log \hat{\psi})\right]$, onde $z_{\alpha / 2}$ é o valor tabelado $\left(100 \frac{\alpha}{2}\right) \%$ da distribuição normal padrão. Estes limites de confiança são então exponenciados para dar um intervalo correspondente para o próprio $\psi$. Observa-se que este método de construir um intervalo de confiança para $\psi$ assegura que ambos os limites sempre serão não-negativos e como uma razão das chances não pode ser negativa, isto é uma condição natural.

Collett (1991) observa que se quisermos obter o próprio erro padrão aproximado de $\hat{\psi}$, isto pode ser feito usando-se o resultado s.e. $(\hat{\psi}) \approx \hat{\psi}$ s.e. $(\log \hat{\psi})$, e também s.e. $(\hat{\psi}) \approx \hat{\psi} \sqrt{\frac{1}{a}+\frac{1}{b}+\frac{1}{c}+\frac{1}{d}}$. Entretanto, por razão dada anteriormente pelo autor, esta expressão não deverá ser usada como uma base para um intervalo de confiança para $\psi$. Porque uma razão de chances igual a 1 será obtida quando as probabilidades de sucesso em dois conjuntos de dados forem iguais. A hipótese nula de que a verdadeira razão da chance é igual à unidade, $\mathrm{H}_{0}: \psi=1$, pode ser testada usando as estatísticas:

$$
Z=\frac{\hat{p}_{1}-\hat{p}_{2}}{\sqrt{\hat{p}(1-\hat{p})\left[\frac{1}{n_{1}}+\frac{1}{n_{2}}\right]}} \text { e } \quad Z^{2}=\frac{n_{1}\left(\hat{p}_{1}-\hat{p}\right)^{2}+n_{2}\left(\hat{p}_{2}-\hat{p}\right)^{2}}{\hat{p}(1-\hat{p})}
$$

ou a estatística $X^{2}$ da equação $X^{2}=\sum_{i=1}^{n} \frac{\left(O_{i}-E_{i}\right)^{2}}{E_{i}}$,

sendo que $\hat{\mathrm{p}}$ é a probabilidade de sucesso, estimada através das probabilidades individuais dos dois conjunto. 


\section{5. - "Outliers"}

Segundo Pregibon (1981), um ajuste de máxima verossimilhança para um modelo de regressão logística é extremamente sensível a respostas afastadas e pontos extremos. Ele desenvolveu medidas de diagnóstico para auxiliar na deteç̧ão de tais observações e na quantificação de seus efeitos sobre vários aspectos do ajuste de máxima verossimilhança. Os elementos do processo de ajustamento, o qual constitui a saída usual de programas estatísticos (parâmetros estimados, erros padrões, resíduos, etc.), foram usados para esta proposta.

Para o modelo de regressão logística, o bloco de construção básica para identificação de "outliers" e pontos influentes será um vetor de resíduos e uma matriz de projeção. Os resíduos mais freqüentemente encontrados, na regressão logística, são os componentes de $\chi^{2}$, dado por

$$
\chi_{i}=\frac{s_{i}}{\sqrt{v_{i i}}}=\frac{\left(y_{i}-n_{i} \hat{p}_{i}\right)}{\sqrt{n_{i} \hat{p}_{i}\left(1-\hat{p}_{i}\right)}}
$$

e os componentes da "deviance",

$$
D=\sum d_{i}^{2}, d_{i}^{2}= \pm \sqrt{2}\left\{1\left(\hat{\theta}_{i} ; y_{i}\right)-1\left(X_{i} \beta_{i} ; y_{i}\right)\right\}^{1 / 2},
$$

onde o mais ou menos é usado de acordo com $\hat{\theta}_{i}>X_{i} \hat{\beta}$ ou $\hat{\theta}_{i}<X_{i} \hat{\beta}$, respectivamente.

Segundo Jennings (1986), a detecção de "outliers" e outros diagnósticos baseados nos resíduos têm sido muito utilizados na regressão linear. Certamente, a regressão logística requer procedimentos para detectar a fragilidade do modelo global e local, isto é, a riqueza do trabalho feito na regressão linear gera guias e sugestões que podem, com cuidado, ser aplicados à regressão logística. Algumas tentativas inovadoras nesta direção têm sido feitas. O autor cita, por exemplo, Tsiatis (1980), Pregibon (1979, 1981), Landwehr, Pregibon e Shoemaker (1984), e Cook e Weisberg (1982). 
Infelizmente, as similaridades que permitem tais técnicas para adaptar à regressão logística parece, além disso, esconder muitas das diferenças.

Duas de tais diferenças são discutidas por ele. A primeira examina o efeito dos eventos de probabilidade pequena sobre estimação na regressão logística. A conclusão simples é que para estimar a probabilidade de sucesso $\mathrm{p}$ em uma área na qual $\mathrm{p}$ é pequena, é preciso observar eventos de probabilidade pequena. Isto sugere que os motivos para detecção dos "outliers" deverão ser cuidadosamente considerados.

A segunda diferença examinada é de acordo com a distribuição dos resíduos. Seja $\mathrm{Y}$ a variável resposta e seja $\mathrm{X}$ um vetor $(\mathrm{p} \times 1)$ de variáveis explanatórias. $\mathrm{Na}$ regressão linear com erros normais, $\mathrm{Y}-\mathrm{E}(\mathrm{Y} / \mathrm{X})$ é normalmente distribuído com média zero e variância $\sigma^{2}$, isto é, a distribuição destes resíduos do modelo verdadeiro não depende das variáveis explanatórias $\mathrm{X}$. Isto não é verdadeiro na regressão logística com dados binários. $\mathrm{Y}$ tem uma distribuição de Bernoulli com probabilidade de sucesso $\mathrm{p}(\mathrm{x})=\mathrm{P}(\mathrm{Y}=1 / \mathrm{x})$. Esta é uma definição razoável de um resíduo para o modelo verdadeiro. Por exemplo, $Y-p(x)$, tem uma distribuição bilateral que depende de $X$ através de $\mathrm{p}(\mathrm{X})$. Ou seja, cada resíduo tem uma única distribuição. Nesta colocação o autor alega que estes resíduos padronizados existem e que são assintoticamente normais ou chi-quadrado e não são apropriados.

A intenção geral do artigo é apontar que aplicações direta das técnicas de regressão linear para regressão logística, não necessariamente produzem ferramentas de diagnósticos úteis. Ou seja, é necessário uma consideração cuidadosa de cada técnica de diagnóstico.

Hosmer \& Lemeshow (1989) consideram duas medidas da diferença entre os valores observados e ajustados que são: o resíduo Pearson e a "deviance" residual. Para um particular modelo de covariáveis, o resíduo Pearson é definido como:

$$
X_{i}=\frac{y_{i}-n_{i} \hat{p}_{i}}{\sqrt{n_{i} \hat{p}_{i}\left(1-\hat{p}_{i}\right)}}
$$


Estes resíduos são conhecidos como resíduos Pearson, uma vez que a soma de seus quadrados é $X^{2}=\sum_{i=1}^{n} X_{i}^{2}$, o qual é a estatística $X^{2}$ de Pearson.

A “deviance" residual é definida como segue:

$$
d\left(y_{i}, \hat{y}_{i}\right)=\operatorname{sinal}\left(y_{i}-\hat{y}_{i}\right)\left[2 y_{i} \ln \left(\frac{y_{i}}{\hat{y}_{i}}\right)+2\left(n_{i}-y_{i}\right) \ln \left(\frac{n_{i}-y_{i}}{n_{i}-\hat{y}_{i}}\right)\right]^{1 / 2}
$$

O resumo estatístico baseado sobre as "deviances" residuais é a "deviance" $D=\sum_{i=1}^{n} d^{2}\left(y_{i}, \hat{y}_{i}\right)$

A distribuição assintótica das estatísticas $\mathrm{X}^{2}$ e $\mathrm{D}$ sobre a suposição que o modelo ajustado é correto em todos os aspectos é suposto ser $\chi^{2} \operatorname{com}[n-(p+1)]$ graus de liberdade.

Collett (1991) apresenta, além dos resíduos dados por Hosmer \& Lemeshow, os resíduos padronizados de Pearson, a "deviance" residual padronizada, os resíduos de verossimilhança e os resíduos de Ascombe.

Para obter os resíduos padronizados de Pearson, basta dividir o resíduo de Pearson por $\sqrt{1-h_{i}}$, sendo que $h_{i}$ é o i-ésimo elemento da matriz $(n \times n)$, $\mathbf{H}=\mathbf{W}^{1 / 2} \mathbf{X}\left(\mathbf{X}^{\prime} \mathbf{W} \mathbf{X}\right)^{-1} \mathbf{X}^{\prime} \mathbf{W}^{1 / 2}$, onde $\mathbf{W}$ é a matriz diagonal $(n \times n)$ dos pesos. Para o modelo logístico linear, o i-ésimo elemento diagonal de $\mathbf{W}$ é $\mathrm{n}_{\mathrm{i}} \hat{\mathrm{p}}_{\mathrm{i}}\left(1-\hat{\mathrm{p}}_{\mathrm{i}}\right)$, ou seja, a variância estimada de $y_{i}$. Logo, $r_{p_{i}}=\frac{y_{i}-n_{i} \hat{p}_{i}}{\sqrt{n_{i} \hat{p}_{i}\left(1-\hat{p}_{i}\right)\left(1-h_{i}\right)}}$

A "deviance" residual padronizada, dada por Collett, é definida como $r_{D_{i}}=\frac{d\left(y_{i}, \hat{y}_{i}\right)}{\sqrt{1-h_{i}}}$, sendo que $d\left(y_{i}, \hat{y}_{i}\right)$ e $h_{i}$ já foram definidos acima.

Os resíduos de verossimilhança são combinações ponderadas dos resíduos padronizados de Pearson e "deviance", dados por: 


$$
r_{L_{i}}=\operatorname{sinal}\left(y_{i}-\hat{y}_{i}\right) \sqrt{h_{i} r_{p_{i}}^{2}+\left(1-h_{i}\right) r_{D_{i}}^{2}}
$$

Segundo o autor, um conjunto de resíduos é melhor interpretado se sua distribuição é conhecida, supondo-se que o modelo ajustado é correto. A distribuição exata de todos os resíduos é muito dificil. Isto sugere que se pudermos encontrar alguma função de observações binomiais, $\mathrm{A}\left(\mathrm{y}_{\mathrm{i}}\right)$, aproximadamente normalmente distribuída, um resíduo apropriado, padronizado para ter aproximadamente variância unitária, deverá ser

$$
r_{A_{i}}=\frac{A\left(y_{i}\right)-A\left(\hat{y}_{i}\right)}{\text { s.e. }\left\{A\left(y_{i}\right)-A\left(\hat{y}_{i}\right)\right\}},
$$

que são conhecidos como resíduos de Ascombe. $\mathrm{A}\left(\mathrm{y}_{\mathrm{i}}\right)$ é uma função da i-ésima observação binomial, $\mathrm{y}_{\mathrm{i}}$, o qual é aproximadamente normalmente distribuída. A função $\mathrm{A}$ apropriada para dados binomiais é dada por:

$$
A(u)=\int_{0}^{u / n_{i}} t^{1 / 3}(1-t)^{-1 / 3} d t, \quad 0 \leq u \leq n_{i}
$$

e o erro padrão no denominador de $r_{\mathrm{A}_{\mathrm{i}}}$ é aproximadamente igual a $\left[\hat{p}_{i}\left(1-\hat{p}_{i}\right)\right]^{1 / 6} \sqrt{\frac{1-h_{i}}{n_{i}}}$. A função $A(u)$ pode ser calculada a partir da função beta incompleta, definida por

$$
I_{z}(\alpha, \beta)=\frac{1}{B(\alpha, \beta)} \int_{0}^{z} t^{\alpha-1}(1-t)^{\beta-1} d t
$$

Após o cálculo dos resíduos, podem-se construir gráficos para visualizar, de maneira mais rápida, o comportamento dos dados e do modelo adotado.

Chatterjee \& Price (1991) mostram que quando os resíduos de regressão não são i.i.d. as estimativas dos coeficientes ainda são não viesados, mas não são melhores no sentido de precisão (variância). Quando existe heterocedasticidade, o problema é resolvido aplicando variações dos mínimos quadrados ponderados. Uma 
importante área para a aplicação da análise de mínimos quadrados ponderados é o ajuste de uma linha de regressão linear quando a variável dependente y é uma proporção. 


\section{METODOLOGIA}

\subsection{Estimação dos Parâmetros}

Seja o modelo logístico linear, dado por:

$$
\operatorname{logit}\left(p_{i}\right)=\ln \left(\frac{p_{i}}{1-p_{i}}\right)=\beta_{0}+\beta_{1} x_{1 i}+\beta_{2} x_{2 i}+\cdots+\beta_{q} x_{q i},
$$

onde

$$
p_{i}=\frac{e^{\beta_{0}+\beta_{1} x_{1 i}+\cdots+\beta_{q} x_{q i}}}{1+e^{\beta_{0}+\beta_{1} x_{1 i}+\cdots+\beta_{q} x_{q i}}} .
$$

Adotando-se

$$
\eta_{i}=\beta_{0}+\beta_{1} x_{1 i}+\cdots+\beta_{q} x_{q i}
$$

tem-se

$$
\mathrm{p}_{\mathrm{i}}=\frac{\mathrm{e}^{\eta_{\mathrm{i}}}}{1+\mathrm{e}^{\eta_{\mathrm{i}}}} .
$$

A função de verossimilhança é dada por:

$$
L(\beta)=\prod_{i=1}^{n}\left(\begin{array}{l}
n_{i} \\
y_{i}
\end{array}\right) p_{i}^{y_{i}}\left(1-p_{i}\right)^{\mathbf{n}_{i}-y_{i}}
$$

e conseqüentemente, o logaritmo da função de verossimilhança é:

$$
\ln L(\beta)=\sum_{i=1}^{n}\left\{\ln \left(\begin{array}{l}
n_{i} \\
y_{i}
\end{array}\right)+y_{i} \ln p_{i}+\left(n_{i}-y_{i}\right) \ln \left(1-p_{i}\right)\right\}
$$




$$
\begin{gathered}
\ln L(\beta)=\sum_{i=1}^{n}\left\{\ln \left(\begin{array}{l}
n_{i} \\
y_{i}
\end{array}\right)+y_{i} \ln p_{i}+n_{i} \ln \left(1-p_{i}\right)-y_{i} \ln \left(1-p_{i}\right)\right\} \\
\ln L(\beta)=\sum_{i=1}^{n}\left\{\ln \left(\begin{array}{l}
n_{i} \\
y_{i}
\end{array}\right)+n_{i} \ln \left(1-p_{i}\right)+y_{i} \ln \frac{p_{i}}{1-p_{i}}\right\},
\end{gathered}
$$

mas,

$$
\begin{gathered}
\ln \left(1-\mathrm{p}_{\mathrm{i}}\right)=\ln \left(1-\frac{\mathrm{e}^{\eta_{\mathrm{i}}}}{1+\mathrm{e}^{\eta_{\mathrm{i}}}}\right)=\ln \left(\frac{1+\mathrm{e}^{\eta_{\mathrm{i}}}-\mathrm{e}^{\eta_{\mathrm{i}}}}{1+\mathrm{e}^{\eta_{\mathrm{i}}}}\right)=\ln \left(\frac{1}{1+\mathrm{e}^{\eta_{\mathrm{i}}}}\right) \\
\ln \left(1-\mathrm{p}_{\mathrm{i}}\right)=\ln 1-\ln \left(1+\mathrm{e}^{\eta_{\mathrm{i}}}\right)=-\ln \left(1+\mathrm{e}^{\eta_{\mathrm{i}}}\right) \\
\ln \left(\frac{\mathrm{p}_{\mathrm{i}}}{1-\mathrm{p}_{\mathrm{i}}}\right)=\ln \left(\frac{\frac{\mathrm{e}^{\eta_{i}}}{1+\mathrm{e}^{\eta_{i}}}}{1-\frac{\mathrm{e}^{\eta_{i}}}{1+\mathrm{e}^{\eta_{i}}}}\right)=\ln \left(\frac{\frac{\mathrm{e}^{\eta_{i}}}{1+\mathrm{e}^{\eta_{i}}}}{\frac{1}{1+\mathrm{e}^{\eta_{i}}}}\right)=\ln \mathrm{e}^{\eta_{i}}=\eta_{\mathrm{i}}
\end{gathered}
$$

portanto,

$$
\ln L(\beta)=\sum_{i=1}^{n}\left\{\ln \left(\begin{array}{l}
n_{i} \\
y_{i}
\end{array}\right)-n_{i} \ln \left(1+e^{n_{i}}\right)+y_{i} \eta_{i}\right\},
$$

onde

$$
\eta_{\mathrm{i}}=\beta_{0}+\beta_{1} \mathrm{x}_{1 \mathrm{i}}+\beta_{2} \mathrm{x}_{2 \mathrm{i}}+\cdots+\beta_{\mathrm{k}} \mathrm{x}_{\mathrm{ki}}
$$

As derivadas parciais em relação aos parâmetros são dadas por:

$$
\begin{gathered}
\frac{\partial \ln L(\beta)}{\partial \beta_{0}}=\sum_{\mathrm{i}=1}^{\mathrm{n}} \mathrm{y}_{\mathrm{i}}-\sum_{\mathrm{i}=1}^{\mathrm{n}} \mathrm{n}_{\mathrm{i}} \frac{1}{1+\mathrm{e}^{\eta_{\mathrm{i}}} \mathrm{e}^{\eta_{\mathrm{i}}}} \\
\frac{\partial \ln \mathrm{L}(\beta)}{\partial \beta_{1}}=\sum_{\mathrm{i}=1}^{\mathrm{n}} \mathrm{y}_{\mathrm{i}} \mathrm{x}_{1 \mathrm{i}}-\sum_{\mathrm{i}=1}^{\mathrm{n}} \mathrm{n}_{\mathrm{i}} \frac{1}{1+\mathrm{e}^{\eta_{\mathrm{i}}}} \mathrm{x}_{1 \mathrm{i}} \mathrm{e}^{\eta_{\mathrm{i}}} \\
\vdots \\
\frac{\partial \ln L(\beta)}{\partial \beta_{\mathrm{q}}}=\sum_{\mathrm{i}=1}^{\mathrm{n}} \mathrm{y}_{\mathrm{i}} \mathrm{x}_{\mathrm{qi}}-\sum_{\mathrm{i}=1}^{\mathrm{n}} \mathrm{n}_{\mathrm{i}} \frac{1}{1+\mathrm{e}^{\eta_{\mathrm{i}}}} \mathrm{x}_{\mathrm{qi}} \mathrm{e}^{\eta_{\mathrm{i}}}
\end{gathered}
$$


Igualando as derivadas a zero, tem-se o seguinte sistema de equações:

$$
\left\{\begin{array}{c}
\sum_{i=1}^{n} y_{i}-\sum_{i=1}^{n} n_{i} \frac{e^{\hat{\beta}_{0}+\hat{\beta}_{1} x_{1 i}+\cdots+\hat{\beta}_{q k} x_{q i}}}{1+e^{\hat{\beta}_{0}+\hat{\beta}_{1} x_{1 i}+\cdots+\hat{\beta}_{q} x_{q i}}}=0 \\
\sum_{i=1}^{n} y_{i} x_{1 i}-\sum_{i=1}^{n} n_{i} x_{1 i} \frac{e^{\hat{\beta}_{0}+\hat{\beta}_{1} x_{1 i}+\cdots+\hat{\beta}_{q} x_{q i}}}{1+e^{\hat{\beta}_{0}+\hat{\beta}_{1} x_{1 i}+\cdots+\hat{\beta}_{q} x_{q i}}}=0 \\
\vdots \\
\vdots \\
\sum_{i=1}^{n} y_{i} x_{q i}-\sum_{i=1}^{n} n_{i} x_{q i} \frac{e^{\hat{\beta}_{0}+\hat{\beta}_{1} x_{11}+\cdots+\hat{\beta}_{q} x_{q i}}}{1+e^{\hat{\beta}_{0}+\hat{\beta}_{1} x_{1 i}+\cdots+\hat{\beta}_{q} x_{q i}}}=0
\end{array}\right.
$$

Assim, o sistema de equações é constituído de $(\mathrm{q}+1)$ equações nãolineares nos parâmetros desconhecidos $\hat{\beta}_{\mathbf{j}}$ que só podem ser resolvidas numericamente.

\subsection{Estimação de Máxima Verossimilhança}

As $\mathrm{q}+1$ derivadas do logaritmo da função de verossimilhança com relação a $\beta_{0}, \beta_{1}, \cdots \beta_{q}$, serão chamadas de escores, e formam um vetor denotado por $\mathbf{u}(\boldsymbol{\beta})$, de dimensão (q x 1), cujo j-ésimo elemento é

$$
\frac{\partial \ln L(\beta)}{\partial \beta_{j}}, \text { para } j=0,1,2, \ldots, q+1 .
$$

Seja a matriz $H(\beta)$, chamada de matriz Hessiana, de dimensão (q $\times q$ ) formada pelas derivadas parciais de segunda ordem de $\ln L(\beta)$, cujo $(j, k)$ - ésimo elémento é dado por é:

$$
\frac{\partial^{2} \ln L(\beta)}{\partial \beta_{j} \partial \beta_{k}}, \quad \text { para } j=1,2, \ldots, q+1 ; k=1,2, \ldots, q+1 .
$$


Considere $\mathbf{u}(\hat{\boldsymbol{\beta}})$, o vetor de escores estimado para a estimativa de máxima verossimilhança $\boldsymbol{\beta}, \hat{\boldsymbol{\beta}}$. Usando uma série de Taylor para expandir $\mathbf{u}(\hat{\boldsymbol{\beta}})$ sobre $\boldsymbol{\beta}^{*}$, onde $\boldsymbol{\beta}^{*}$ é próximo a $\hat{\boldsymbol{\beta}}$, tem-se que:

$$
\mathbf{u}(\hat{\boldsymbol{\beta}}) \approx \mathbf{u}\left(\boldsymbol{\beta}^{*}\right)+\mathbf{H}\left(\boldsymbol{\beta}^{*}\right)\left(\hat{\boldsymbol{\beta}}-\boldsymbol{\beta}^{*}\right) .
$$

Por definição, as estimativas de máxima verossimilhança dos $\beta$ `s devem satisfazer às equações:

$$
\left.\frac{\partial \ln L(\beta)}{\partial \beta_{j}}\right|_{\hat{\beta}}=0, \text { para } j=1,2, \ldots, q,
$$

e também $\mathbf{u}(\hat{\boldsymbol{\beta}})=\mathbf{0}$

Da equação (2), segue então que

$$
\mathbf{u}(\hat{\boldsymbol{\beta}}) \approx \mathbf{u}\left(\boldsymbol{\beta}^{*}\right)+\mathbf{H}\left(\boldsymbol{\beta}^{*}\right)\left(\hat{\boldsymbol{\beta}}-\boldsymbol{\beta}^{*}\right)
$$

ou seja,

$$
\mathbf{H}^{-1}\left(\boldsymbol{\beta}^{*}\right) \mathbf{u}(\hat{\boldsymbol{\beta}}) \approx \mathbf{H}^{-1}\left(\boldsymbol{\beta}^{*}\right) \mathbf{u}\left(\boldsymbol{\beta}^{*}\right)+\mathbf{H}^{-1}\left(\boldsymbol{\beta}^{*}\right) \mathbf{H}\left(\boldsymbol{\beta}^{*}\right)\left(\hat{\boldsymbol{\beta}}-\boldsymbol{\beta}^{*}\right)
$$

$\operatorname{como} \mathbf{u}(\hat{\boldsymbol{\beta}})=\mathbf{0}$,

$$
\begin{gathered}
\mathbf{0} \approx \mathbf{H}^{-1}\left(\boldsymbol{\beta}^{*}\right) \mathbf{u}\left(\boldsymbol{\beta}^{*}\right)+\hat{\boldsymbol{\beta}}-\boldsymbol{\beta}^{*} \\
\hat{\boldsymbol{\beta}} \approx \boldsymbol{\beta}^{*}-\mathbf{H}^{-1}\left(\boldsymbol{\beta}^{*}\right) \mathbf{u}\left(\boldsymbol{\beta}^{*}\right)
\end{gathered}
$$

sugerindo um método iterativo para estimação de $\hat{\beta}$, no qual a estimativa de $\beta$ para $o$ $(\mathrm{r}+1)$-ésimo ciclo de iteração é dado por:

$$
\hat{\boldsymbol{\beta}}_{\mathrm{r}+\mathbf{1}}=\hat{\boldsymbol{\beta}}_{\mathrm{r}}-\mathbf{H}^{-1}\left(\hat{\boldsymbol{\beta}}_{\mathrm{r}}\right) \mathbf{u}\left(\hat{\boldsymbol{\beta}}_{\mathrm{r}}\right)
$$

para $\mathrm{r}=0,1,2, \ldots$, onde $\hat{\boldsymbol{\beta}}_{0}$ é um vetor de estimativas iniciais de $\boldsymbol{\beta}$.

Esse é o procedimento Newton-Raphson para a obtenção de estimadores de máxima verossimilhança de $\beta$.

Se trocarmos a matriz $\mathbf{H}(\boldsymbol{\beta})$ pela matriz dos valores esperados de derivadas parciais de segunda ordem da o logaritmo da função de verossimilhança, tem-se o algoritmo, usado pelo GLIM ("Generalised Linear Interactive Modelling"). Quando 
esta matriz é multiplicada por -1 , obtém-se a matriz de informação, que é a matriz cujo (j, k)-ésimo elemento é:

$$
-\mathrm{E}\left\{\frac{\partial^{2} \ln \mathrm{L}(\beta)}{\partial \beta_{j} \partial \beta_{\mathrm{k}}}\right\}=\mathrm{E}\left\{\frac{\partial \ln \mathrm{L}(\beta)}{\partial \beta_{j}} \frac{\partial \ln \mathrm{L}(\beta)}{\partial \beta_{\mathrm{k}}}\right\}
$$

para $\mathrm{j}=1,2, \ldots, \mathrm{q} ; \mathrm{k}=1,2, \ldots, \mathrm{q}$. Esta matriz é denotada por $\mathbf{I}(\boldsymbol{\beta})$, e tem um papel particularmente importante na estimação de máxima verossimilhança, uma vez que a inversa de $\mathbf{I}(\boldsymbol{\beta})$ é a matriz de variâncias e covariâncias das estimativas de máxima verossimilhança dos parâmetros.

Usando a matriz de informação no método iterativo definido pela equação (3), tem-se

$$
\hat{\boldsymbol{\beta}}_{\mathrm{r}+1}=\hat{\boldsymbol{\beta}}_{\mathrm{r}}+\mathbf{I}^{-1}\left(\hat{\boldsymbol{\beta}}_{\mathbf{r}}\right) \mathbf{u}\left(\hat{\boldsymbol{\beta}}_{\mathbf{r}}\right)
$$

O procedimento iterativo baseado na equação (4) é conhecido como método de escore de Fisher, da qual é obtida é obtida uma estimativa da matriz de variâncias e covariâncias assintóticas, $\mathbf{I}^{-1}(\hat{\boldsymbol{\beta}})$.

Ambos os procedimentos Newton-Raphson e escore de Fisher convergem para a estimativa de máxima verossimilhança de $\boldsymbol{\beta}$. Porém, os erros padrões dos parâmetros estimados, obtidos como as raízes quadradas dos elementos diagonais de $-\mathbf{H}^{-1}(\hat{\boldsymbol{\beta}})$ ou $\mathbf{I}^{-1}(\hat{\boldsymbol{\beta}})$, podem se diferir em algumas situações.

Quando estes algoritmos são usados para ajustar um modelo logístico linear para dados binomiais, tem-se que

$$
\mathbf{I}^{-1}(\hat{\boldsymbol{\beta}})=-\mathbf{H}^{-1}(\hat{\boldsymbol{\beta}}) \text {. }
$$

Além disso, os dois algoritmos darão os mesmos erros padrões dos parâmetros estimados.

Para outras transformações de probabilidade de resposta, as matrizes de variâncias e covariâncias obtidas poderão deferir nos dois procedimentos. Segundo Collett (1991), em grandes amostras esta diferença será pequena, mas em pequenas amostras esta diferença pode ser marcante. 


\subsection{Ajuste de um Modelo Linear Generalizado a Dados Binomiais}

Suponha que $\mathrm{n}$ observações binomiais independentes $\mathrm{y}_{1}, \mathrm{y}_{2}, \ldots, \mathrm{y}_{\mathrm{n}}$ são tais que a i-ésima observação, $i=1,2, \ldots, n$, tem distribuição binomial com parâmetros $n_{i} e$ $\mathrm{p}_{\mathrm{i}}$. Também suponha que o valor transformado da probabilidade de resposta para a iésima observação está relacionado a uma combinação linear de q variáveis explanatórias, isto é,

$$
g\left(p_{i}\right)=\beta_{1} x_{1 i}+\cdots+\beta_{q} x_{q i}
$$

onde $g(p)$ pode ser a transformação logística de $p$, o "probit" de $p$ ou a transformação complemento log-log de p. O componente linear deste modelo será denotado por $\eta_{i}$, também que $\mathrm{g}\left(\mathrm{p}_{\mathrm{i}}\right)=\eta_{\mathrm{i}}$. A função $\mathrm{g}$ é a função de ligação de um modelo linear generalizado.

Para ajustar este modelo usando o método iterativo da equação (4), são necessárias expressões para $\mathbf{u}(\boldsymbol{\beta})$ e $\mathbf{I}(\boldsymbol{\beta})$.

O logaritmo da função de verossimilhança para $\mathrm{n}$ observações binomiais é dada por

$$
\ln L(\beta)=\sum_{i=1}^{n}\left\{\ln \left(\begin{array}{l}
n_{i} \\
y_{i}
\end{array}\right)+y_{i} \ln p_{i}+\left(n_{i}-y_{i}\right) \ln \left(1-p_{i}\right)\right\},
$$

e

$$
\frac{\partial \ln L(\beta)}{\partial \beta_{j}}=\sum_{i=1}^{n}\left(\frac{\partial \ln L}{\partial p_{i}} \frac{\partial p_{i}}{\partial \eta_{i}} \frac{\partial \eta_{i}}{\partial \beta_{j}}\right)
$$

tem-se que

$$
\begin{gathered}
\frac{\partial \ln L(\beta)}{\partial p_{i}}=\sum_{i=1}^{n}\left(y_{i} \frac{1}{p_{i}}-n_{i} \frac{1}{1-p_{i}}+y_{i} \frac{1}{1-p_{i}}\right) \\
\frac{\partial \ln L(\beta)}{\partial p_{i}}=\sum_{i=1}^{n}\left(\frac{y_{i}}{p_{i}}-\frac{n_{i}-y_{i}}{1-p_{i}}\right) \\
\frac{\partial \ln L(\beta)}{\partial p_{i}}=\sum_{i=1}^{n}\left(\frac{y_{i}-y_{i} p_{i}-n_{i} p_{i}+y_{i} p_{i}}{p_{i}\left(1-p_{i}\right)}\right)
\end{gathered}
$$




$$
\frac{\partial \ln L(\beta)}{\partial p_{i}}=\sum_{i=1}^{n}\left(\frac{y_{i}-n_{i} p_{i}}{p_{i}\left(1-p_{i}\right)}\right)
$$

Também, como

$$
\eta_{i}=\beta_{1} x_{1 i}+\beta_{2} x_{2 i}+\cdots+\beta_{q} x_{q i}
$$

tem-se,

$$
\frac{\partial \eta_{\mathrm{i}}}{\partial \beta_{\mathrm{j}}}=\mathrm{x}_{\mathrm{ji}}
$$

e escrevendo $g^{\prime}\left(p_{i}\right)$ para $\frac{\partial \eta_{i}}{\partial p_{i}}$

$$
\frac{\partial \ln L(\beta)}{\partial \beta_{j}}=\sum_{i=1}^{n}\left\{\frac{y_{i}-n_{i} p_{i}}{p_{i}\left(1-p_{i}\right)} \frac{1}{g^{\prime}\left(p_{i}\right)} x_{j i}\right\}
$$

Seja

$$
y_{i}^{*}=\frac{\left(y_{i}-n_{i} p_{i}\right) g^{\prime}\left(p_{i}\right)}{n_{i}}
$$

e

$$
w_{i}=\frac{n_{i}}{\left\{p_{i}\left(1-p_{i}\right)\left[g^{\prime}\left(p_{i}\right)\right]^{2}\right\}},
$$

também que

$$
\frac{\partial \ln L(\beta)}{\partial \beta_{j}}=\sum_{i=1}^{n} w_{i} y_{i}^{*} x_{j i}
$$

Se $\mathbf{X}$ é a matriz de valores ( $\mathrm{n} \times \mathrm{q}$ ) das q variáveis explanatórias, $\mathbf{W}$ é a matriz diagonal $(\mathrm{n} \times \mathrm{n})$ cujo i-ésimo elemento diagonal é $\mathrm{w}_{\mathrm{i}}$, e $\mathrm{y}^{*}$ é o vetor $(\mathrm{n} \times 1)$ cujo i-ésimo componente é $\mathrm{y}_{\mathrm{i}}^{*}$, o vetor de escores eficientes, $\mathbf{u}(\boldsymbol{\beta})$, pode ser escrito como

$$
\mathbf{u}(\boldsymbol{\beta})=\mathbf{X}^{\mathbf{}} \mathbf{W} \mathbf{y}^{*} .
$$

Para obter o (j, k)-ésimo elemento da matriz informação, usa-se o resultado padrão que: 


$$
-E\left\{\frac{\partial^{2} \ln L(\beta)}{\partial \beta_{j} \partial \beta_{k}}\right\}=E\left(\frac{\partial \ln L(\beta)}{\partial \beta_{j}} \frac{\partial \ln L(\beta)}{\partial \beta_{k}}\right) .
$$

Então, o (j, k)-ésimo elemento de $\mathbf{I}(\boldsymbol{\beta})$ é dado por:

$$
E\left\{\sum_{i=1}^{n} \frac{y_{i}-n_{i} p_{i}}{p_{i}\left(1-p_{i}\right)} \frac{1}{g^{\prime}\left(p_{i}\right)} x_{j i} \sum_{i^{\prime}=1}^{n} \frac{y_{i^{\prime}}-n_{i^{\prime}} p_{i^{\prime}}}{p_{i^{\prime}}\left(1-p_{i^{\prime}}\right)} \frac{1}{g^{\prime}\left(p_{i^{\prime}}\right)} x_{k i^{\prime}}\right\}
$$

Também,

$$
E\left[\left(y_{i}-n_{i} p_{i}\right)\left(y_{i^{\prime}}-n_{i^{\prime}} p_{i^{\prime}}\right)\right]=\operatorname{Cov}\left(y_{i}, y_{i^{\prime}}\right)=0, \text { para } i \neq i^{\prime},
$$

uma vez que as observações são independentes, para $i=i^{\prime}$, esta esperança resulta em

$$
E\left[\left(y_{i}-n_{i} p_{i}\right)^{2}\right]=\operatorname{Var}\left(y_{i}\right)=n_{i} p_{i}\left(1-p_{i}\right)
$$

Então, segue que:

$$
\begin{aligned}
-E\left\{\frac{\partial^{2} \ln L(\beta)}{\partial \beta_{j} \partial \beta_{k}}\right\} & =E\left\{\sum_{i=1}^{n} \frac{y_{i}-n_{i} p_{i}}{p_{i}\left(1-p_{i}\right)} \frac{1}{g^{\prime}\left(p_{i}\right)} x_{j i} \sum_{i=1}^{n} \frac{y_{i}-n_{i} p_{i}}{p_{i}\left(1-p_{i}\right)} \frac{1}{g^{\prime}\left(p_{i}\right)} x_{k i}\right\} \\
& =E\left\{\sum_{i=1}^{n} \frac{\left(y_{i}-n_{i} p_{i}\right)^{2}}{\left[p_{i}\left(1-p_{i}\right)\right]^{2}} \frac{x_{j i} x_{k i}}{\left[g^{\prime}\left(p_{i}\right)\right]^{2}}\right\} \\
& =\sum_{i=1}^{n} \frac{E\left[\left(y_{i}-n_{i} p_{i}\right)^{2}\right] x_{j i} x_{k i}}{\left[p_{i}\left(1-p_{i}\right)\right]^{2}\left[g^{\prime}\left(p_{i}\right)\right]^{2}} \\
& =\sum_{i=1}^{n} \frac{n_{i} p_{i}\left(1-p_{i}\right) x_{j i} x_{k i}}{\left[p_{i}\left(1-p_{i}\right)\right]^{2}\left[g^{\prime}\left(p_{i}\right)\right]^{2}} \\
-E\left\{\frac{\partial^{2} \ln L(\beta)}{\partial \beta_{j} \partial \beta_{k}}\right\} & =\sum_{i=1}^{n} \frac{n_{i}}{\left[p_{i}\left(1-p_{i}\right)\right]\left[g^{\prime}\left(p_{i}\right)\right]^{2}} x_{j i} x_{k i}
\end{aligned}
$$

e, na forma matricial, a matriz informação é $\mathbf{X}^{\mathbf{X}} \mathbf{W} \mathbf{X}$. 
Substituindo para $\mathbf{u}(\hat{\boldsymbol{\beta}})$ e $\mathbf{I}(\hat{\boldsymbol{\beta}})$ na equação (4), encontra-se que a estimativa de $\boldsymbol{\beta}$ para a $(\mathrm{r}+1)$-ésima iteração é

$$
\hat{\boldsymbol{\beta}}_{\mathrm{r}+1}=\hat{\boldsymbol{\beta}}_{\mathrm{r}}+\left(\mathbf{X}^{\prime} \mathbf{W}_{\mathrm{r}} \mathbf{X}\right)^{-1} \mathbf{X}^{\prime} \mathbf{W}_{\mathrm{r}} \mathbf{y}_{\mathrm{r}}^{*}
$$

onde $\mathbf{W}_{\mathrm{r}}$ é a matriz diagonal dos valores de $\mathrm{w}_{\mathrm{i}}$ para o r-ésimo ciclo e $\mathbf{y}_{\mathrm{r}}^{*}$, é o vetor de valores de $\mathrm{y}_{\mathrm{i}}^{*}$ para o r-ésimo ciclo. Então,

$$
\begin{aligned}
& \hat{\beta}_{r+1}=\left(X^{\prime} W_{r} X\right)^{-1}\left[X^{\prime} W_{r}\left(X \hat{\beta}_{r}+y_{r}^{*}\right)\right] \\
& \hat{\beta}_{r+1}=\left(X^{\prime} W_{r} X\right)^{-1} X^{\prime} W_{r} z_{r}
\end{aligned}
$$

onde $\mathbf{z}_{\mathbf{r}}=\mathbf{X} \hat{\boldsymbol{\beta}}_{\mathbf{r}}+\mathbf{Y}_{\mathbf{r}}^{*}=\hat{\eta}_{\mathbf{r}}+\mathbf{y}_{\mathbf{r}}^{*}, \hat{\eta}_{\mathbf{r}}$ é o vetor de valores do preditor linear para o r-ésimo ciclo da iteração.

Conseqüentemente, $\hat{\boldsymbol{\beta}}_{\mathbf{r}+\mathbf{1}}$ é obtido pelos valores regressores da variável dependente ajustada $\mathbf{z}_{\mathbf{r}}$, cujo i-ésimo elemento é

$$
\hat{\eta}_{\mathrm{ir}}+\frac{\left(\mathrm{y}_{\mathrm{i}}-\mathrm{n}_{\mathrm{i}} \hat{\mathrm{p}}_{\mathrm{ir}}\right) \mathrm{g}^{\prime}\left(\hat{\mathrm{p}}_{\mathrm{ir}}\right)}{\mathrm{n}_{\mathrm{i}}}
$$

sobre as $\mathrm{q}$ variáveis explanatórias $\mathrm{x}_{1}, \mathrm{x}_{2}, \ldots, \mathrm{x}_{\mathrm{q}}$, usando pesos $\mathrm{w}_{\mathrm{ir}}$, onde

$$
\mathrm{w}_{\mathrm{ir}}=\frac{\mathrm{n}_{\mathrm{i}}}{\hat{\mathrm{p}}_{\mathrm{ir}}\left(1-\hat{\mathrm{p}}_{\mathrm{ir}}\right)\left[\mathrm{g}^{\prime}\left(\hat{\mathrm{p}}_{\mathrm{ir}}\right)\right]^{2}}
$$

e $\hat{\mathrm{p}}_{\mathrm{ir}}, \hat{\eta}_{\mathrm{ir}}$ são estimados de valores de $\hat{\boldsymbol{\beta}}$ após o r-ésimo ciclo da iteração, $\hat{\boldsymbol{\beta}}_{\text {r- }}$

Para começar o processo iterativo, as estimativas iniciais de $p_{i}$ podem ser são dadas por:

$$
\hat{p}_{i 0}=\frac{y_{i}+0.5}{n_{i}+1}
$$

Os pesos iniciais são, então,

$$
\mathrm{w}_{\mathrm{i} 0}=\frac{\mathrm{n}_{\mathrm{i}}}{\hat{\mathrm{p}}_{\mathrm{i} 0}\left(1-\hat{\mathrm{p}}_{\mathrm{i} 0}\right)\left[\mathrm{g}^{\prime}\left(\hat{\mathrm{p}}_{\mathrm{i} 0}\right)\right]^{2}},
$$

e os valores iniciais da variável dependente pode ser considerado como

$$
z_{i 0}=\hat{\eta}_{i 0}=g\left(\hat{p}_{i 0}\right) \text {. }
$$


Os valores de $\mathrm{z}_{\mathrm{i} 0}$ são, então, estimados para as $\mathrm{q}$ variáveis explanatórias $\mathrm{x}_{1}, \mathrm{x}_{2}, \ldots, \mathrm{x}_{\mathbf{q}}$ usando o método de mínimos quadrados ponderados, com pesos $\mathrm{w}_{\mathrm{i} 0}$.

Os resultados estimados dos coeficientes de $x_{1}, x_{2}, \ldots, x_{q}$, dados por $\hat{\beta}_{1}$, conduzem a estimativas corrigidas do preditor linear

$$
\hat{\eta}_{\mathrm{i} 1}=\sum \hat{\beta}_{\mathrm{j} 1} \mathbf{x}_{\mathrm{ji}},
$$

probabilidades ajustadas corrigidas

$$
\hat{\mathrm{p}}_{\mathrm{i} 1}=\mathrm{g}^{-1}\left(\hat{\eta}_{\mathrm{i} 1}\right)
$$

pesos ajustados

$$
\mathrm{w}_{\mathrm{i} 1}=\frac{\mathrm{n}_{\mathrm{i}}}{\hat{\mathrm{p}}_{\mathrm{i} 1}\left(1-\hat{\mathrm{p}}_{\mathrm{i} 1}\right)\left[\mathrm{g}^{\prime}\left(\hat{\mathrm{p}}_{\mathrm{i} 1}\right)\right]^{2}},
$$

e valores ajustados da variável dependente ajustada

$$
z_{i 1}=\hat{\eta}_{i 1}+\frac{\left(y_{i}-n_{i} \hat{p}_{i 1}\right) g^{\prime}\left(\hat{p}_{i 1}\right)}{n_{i}}
$$

Os valores ajustados dos parâmetros estimados, $\hat{\boldsymbol{\beta}}_{2}$, são obtidos pela regressão de $z_{i 1}$ sobre as variáveis explanatórias, usando pesos $w_{i 1}$, e assim por diante. Para cada etapa do processo pode ser calculada a "deviance" e a iteração continua até que a mudança na "deviance" em ciclos sucessivos seja suficientemente pequena.

\subsection{Interpretação dos Parâmetros de Regressão}

Os coeficientes de regressão têm um papel similar aos dos coeficientes de regressão parcial na regressão linear múltipla. Suponha dois indivíduos com diferentes valores das variáveis independentes, como mostrado na tabela abaixo, onde a j-ésima variável independente é uma variável binária: 
Tabela 3 - Presença ou Ausência das Variáveis independentes, ou fatores de exposição, nos individuos $\mathrm{A}$ e $\mathrm{B}$

\begin{tabular}{ccccccccc}
\hline & \multicolumn{7}{c}{ Variável Independente } \\
\cline { 2 - 8 } Indivíduo & 1 & 2 & $\ldots$ & $\mathrm{j}-1$ & $\mathbf{j}$ & $\mathrm{j}+1$ & $\ldots$ & $\mathrm{k}$ \\
\hline $\mathrm{A}$ & $\mathrm{x}_{1}$ & $\mathrm{x}_{2}$ & $\ldots$ & $\mathrm{x}_{\mathrm{j}-1}$ & $\mathbf{1}$ & $\mathrm{x}_{\mathrm{j}+1}$ & $\ldots$ & $\mathrm{x}_{\mathrm{k}}$ \\
$\mathrm{B}$ & $\mathrm{x}_{1}$ & $\mathrm{x}_{2}$ & $\ldots$ & $\mathrm{x}_{\mathrm{j}-1}$ & $\mathbf{0}$ & $\mathrm{x}_{\mathrm{j}+1}$ & $\ldots$ & $\mathrm{x}_{\mathrm{k}}$ \\
\hline
\end{tabular}

Referindo-se às variáveis independentes como fatores de exposição, ter-seá, então, que os indivíduos A e B estão expostos a todos os fatores de risco do modelo, exceto para j-ésima variável expositora, onde o indivíduo $\mathrm{A}$ é exposto e o indivíduo $\mathrm{B}$ não é exposto. $\mathrm{O}$ "logit" da probabilidade de sucesso para indivíduos $\mathrm{A}$ e $\mathrm{B}$, denotado por "logit" $\left(\mathbf{p}_{\mathrm{A}}\right)$ e "logit" $\left(\mathbf{p}_{\mathrm{B}}\right)$ são dados por:

$$
\begin{aligned}
& \operatorname{logit}\left(p_{A}\right)=\alpha+\beta_{1} x_{1}+\cdots+\beta_{j-1} x_{j-1}+\beta_{j}(1)+\beta_{j+1} x_{j+1}+\cdots+\beta_{k} x_{k} \\
& \operatorname{logit}\left(p_{B}\right)=\alpha+\beta_{1} x_{1}+\cdots+\beta_{j-1} x_{j-1}+\beta_{j}(0)+\beta_{j+1} x_{j+1}+\cdots+\beta_{k} x_{k}
\end{aligned}
$$

Subtraindo o "logit" $\left(\mathrm{p}_{A}\right)$ do "logit" $\left(\mathrm{p}_{B}\right)$, obtém-se

$$
\log i t\left(p_{A}\right)-\log \operatorname{it}\left(p_{B}\right)=\beta_{j} \text {. }
$$

Entretanto,

$$
\operatorname{logit}\left(\mathrm{p}_{\mathrm{A}}\right)=\ln \frac{\mathrm{p}_{\mathrm{A}}}{1-\mathrm{p}_{\mathrm{A}}} \quad \text { e } \quad \log \operatorname{it}\left(\mathrm{p}_{\mathrm{B}}\right)=\ln \frac{\mathrm{p}_{\mathrm{B}}}{1-\mathrm{p}_{\mathrm{B}}} .
$$

Logo, tem-se que

$$
\ln \frac{p_{A}}{1-p_{A}}-\ln \frac{p_{B}}{1-p_{B}}=\beta_{j}
$$

ou

$$
\ln \left[\frac{p_{A} /\left(1-p_{A}\right)}{p_{B} /\left(1-p_{B}\right)}\right]=\beta_{j}
$$


Se for considerado o antilogaritmo, então ter-se-á

$$
\frac{p_{A} /\left(1-p_{A}\right)}{p_{B} /\left(1-p_{B}\right)}=e^{\beta_{j}} \text {. }
$$

Contudo, a chance em favor do sucesso para o indivíduo A (denotado por 0) é dado por

$$
\psi_{\mathrm{A}}=\frac{\mathrm{p}_{\mathrm{A}}}{1-\mathrm{p}_{\mathrm{A}}} .
$$

Similarmente

$$
\psi_{\mathrm{B}}=\frac{\mathrm{p}_{\mathrm{B}}}{1-\mathrm{p}_{\mathrm{B}}} .
$$

Assim, tem-se que a razão das chances será dada por:

$$
\frac{\psi_{\mathrm{A}}}{\psi_{\mathrm{B}}}=\mathrm{e}^{\beta_{\mathrm{j}}}
$$

Em outras palavras, a chance em favor da doença para o indivíduo A dividida pela chance em favor da doença para o indivíduo $B$ é igual a $\mathrm{e}^{\beta_{j}}$. Contudo, pode-se pensar na

$$
\frac{\psi_{\mathrm{A}}}{\psi_{\mathrm{B}}}=\psi
$$

como a razão das chances relativa à doença para a j-ésima variável exposição para dois indivíduos, um deles é exposto à j-ésima variável (indivíduo A) e o outro não é exposto (indivíduo B), sendo que os fatores de riscos considerados no modelo são os mesmos para ambos os indivíduos. Ou seja, esta razão das chances é uma razão de chances relativa à doença para a j-ésima variável de exposição, ajustada para os níveis de todos os outros fatores no corrente modelo. A razão de chances, $\psi$, é usualmente o parâmetro de interesse na regressão logística devido a sua facilidade de interpretação. Entretanto, sua estimativa, $\hat{\psi}$, tenderá a ter uma distribuição que é assimétrica.

Por isso, influências são usualmente baseadas na distribuição amostral de $\ln (\hat{\psi})=\hat{\beta}_{1}$, que tende a seguir uma distribuição assintoticamente normal para tamanhos amostrais menores. 
Um intervalo de confiança estimado de $100 \times(1-\alpha) \%$ para a razão das chances pode ser obtida calculando os pontos extremos de um intervalo de confiança para o coeficiente $\beta_{\mathrm{i}}$, e então exponenciando estes valores. Em geral, os pontos extremos são dados pela expressão:

$$
\mathrm{e}^{\hat{\hat{\beta}_{i} \pm Z_{\alpha / 2} \text { xs.e.( }\left(\beta_{j}\right)}}
$$

Para saber se os parâmetros são estatisticamente significativos, aplica-se o teste da razão de verossimilhança, dado pela diferença:

$$
-2 \ln \hat{\mathrm{L}}_{\mathrm{A}}-\left(-2 \ln \hat{\mathrm{L}}_{\mathrm{B}}\right)=-2 \ln \left[\frac{\hat{\mathrm{L}}_{\mathrm{A}}}{\hat{\mathrm{L}}_{\mathrm{B}}}\right]
$$

cuja hipótese a ser testada é a de que:

$$
\mathrm{H}_{0}: \beta_{\mathrm{j}}=0 \quad \text { vs } \quad \mathrm{H}_{\mathrm{A}}: \boldsymbol{\beta}_{\mathrm{j}} \neq 0
$$

ou, equivalentemente:

$$
\mathrm{H}_{0} \quad: \quad \psi=\mathrm{e}^{\beta_{j}}=1 \quad \text { vs } \quad \mathrm{H}_{\mathrm{A}} \quad: \quad \psi=\mathrm{e}^{\beta_{\mathrm{j}}} \neq 1 .
$$

O número de graus de liberdade é a diferença entre o número de parâmetros nos dois modelos.

\subsection{A "Deviance" para Dados Binários}

Após obterem-se as estimativas dos parâmetros, é necessário verificar se os valores ajustados da variável resposta sobre o modelo retrata bem os valores observados.

No caso de dados binários, onde $n_{i}=1$, para $i=1,2, \ldots, n$, a "deviance" depende somente das probabilidades de sucessos ajustadas $\mathrm{p}_{\mathrm{i}}$, porém, não 
informa sobre a qualidade de ajuste de um modelo. Para ver isto, a verossimilhança para $n$ observações binárias, como uma função de $\beta$ parâmetros, é

$$
L(\beta)=\prod_{i} p_{i}^{y_{i}}\left(1-p_{i}\right)^{1-y_{i}},
$$

e a função do logaritmo de verossimilhança maximizada sobre algum modelo corrente é:

$$
\ln \hat{\mathrm{L}}_{\mathrm{C}}=\sum\left\{\mathrm{y}_{\mathrm{i}} \ln \hat{\mathrm{p}}_{\mathrm{i}}+\left(1-\mathrm{y}_{\mathrm{i}}\right) \ln \left(1-\hat{\mathrm{p}}_{\mathrm{i}}\right)\right\}
$$

Para o modelo completo, $\hat{p}_{i}=y_{i}$, e uma vez que $y_{i} \ln y_{i}$ e $\left(1-y_{i}\right) \ln \left(1-y_{i}\right)$ são ambos iguais a zero para somente dois valores possíveis de $y_{i}, 0$ e $1, \ln \hat{L}_{f}=0$. A "deviance" para dados binários resulta então:

$$
\begin{gathered}
D=-2 \sum\left\{y_{i} \ln \hat{p}_{i}+\left(1-y_{i}\right) \ln \left(1-\hat{p}_{i}\right)\right\} \\
D=-2 \sum\left\{y_{i} \ln \hat{p}_{i}+\ln \left(1-\hat{p}_{i}\right)-y_{i} \ln \left(1-\hat{p}_{i}\right)\right\} \\
D=-2 \sum\left\{y_{i} \ln \frac{\hat{p}_{i}}{\left(1-\hat{p}_{i}\right)}+\ln \left(1-\hat{p}_{i}\right)\right\}
\end{gathered}
$$

Diferenciando $\ln L(\beta)$ em relação ao j-ésimo parâmetro, $\beta_{\mathrm{j}}$, tem-se:

$$
\frac{\partial \ln L(\beta)}{\partial \beta_{j}}=\sum_{i}\left\{\frac{y_{i}}{p_{i}}-\frac{1-y_{i}}{1-p_{i}}\right\} p_{i}\left(1-p_{i}\right) x_{j i},
$$

do qual

$$
\begin{gathered}
\sum_{j} \beta_{j} \frac{\partial \ln L(\beta)}{\partial \beta_{j}}=\sum_{i}\left\{\frac{y_{i}-y_{i} p_{i}-p_{i}+y_{i} p_{i}}{p_{i}\left(1-p_{i}\right)}\right\} p_{i}\left(1-p_{i}\right) \sum_{j} \beta_{j} x_{j i} \\
\sum_{j} \beta_{j} \frac{\partial \ln L(\beta)}{\partial \beta_{j}}=\sum_{i}\left(y_{i}-p_{i}\right) \sum_{j} \beta_{j} x_{j i}
\end{gathered}
$$




$$
\sum_{j} \beta_{j} \frac{\partial \ln L(\beta)}{\partial \beta_{j}}=\sum_{i}\left(y_{i}-p_{i}\right) \ln \frac{p_{i}}{1-p_{i}}
$$

Pelo fato de $\hat{\beta}$ ser a estimativa de máxima verossimilhança de $\beta$, a derivada do lado esquerdo é igual a zero para $\hat{\boldsymbol{\beta}}$. Conseqüentemente, as probabilidades ajustadas $\hat{\mathrm{p}}_{\mathrm{i}}$ devem satisfazer à equação:

$$
\sum_{i}\left(y_{i}-\hat{p}_{i}\right) \log \text { it } \hat{p}_{i}=0
$$

e também

$$
\sum_{i} y_{i} \log i t \hat{p}_{i}=\sum_{i} \hat{p}_{i} \log i t \hat{p}_{i}
$$

Substituindo em (5), tem-se que:

$$
D=-2 \sum\left\{\hat{p}_{i} \log \text { it } \hat{p}_{i}+\ln \left(1-\hat{p}_{i}\right)\right\}
$$

Essa "deviance" depende das observações binárias y $y_{i}$ somente através das probabilidades ajustadas $\hat{\mathrm{p}}_{\mathrm{i}}$ e também nada diz sobre a concordância entre as observações e suas correspondentes probabilidades ajustadas. Conseqüentemente, a "deviance" no ajuste de um modelo a dados de resposta binária não pode ser usada como uma medida resumo da qualidade de ajuste de um modelo.

Para se avaliar a significância de uma variável independente, compara-se o valor de $\mathrm{D}$ com e sem a variável independente na equação. $\mathrm{A}$ mudança em $\mathrm{D}$ devido à inclusão de uma variável independente no modelo, é obtida como segue:

$$
\mathrm{G}=\mathrm{D} \text { (para o modelo sem a variável) }-\mathrm{D} \text { (para o modelo com a variável) }
$$

logo,

$$
G=-2 \ln \left[\frac{\text { verossimilhanca sem a variavel }}{\text { verossimilhanca com a variavel }}\right]
$$

A diferença entre as "deviances" será comparada com o valor de $\chi^{2}$ de tabela, com tantos graus de liberdade quanto for o número de variáveis diferentes nos dois modelos comparados, para verificar se a inclusão da variável no modelo é significativa ao nível $\alpha$ de significância. 


\subsection{Cálculo de "Outliers"}

Muitas vezes, após estimar os parâmetros e verificar que o modelo foi ajustado corretamente é necessário verificar se não existem pontos discrepantes ou extremos no conjunto de dados. Para verificar isto, usam-se os resíduos padronizados de Pearson, cuja fórmula é dada por:

$$
r_{p_{i}}=\frac{y_{i}-n_{i} \hat{p}_{i}}{\sqrt{n_{i} \hat{p}_{i}\left(1-\hat{p}_{i}\right)\left(1-h_{i}\right)}}
$$

onde $h_{i}$ é o i-ésimo elemento da matriz ( $n \times n$ ),

$$
\mathbf{H}=\mathbf{W}^{1 / 2} \mathbf{X}\left(\mathbf{X}^{\prime} \mathbf{W X}\right)^{-1} \mathbf{X}^{\prime} \mathbf{W}^{1 / 2}
$$

sendo que $\mathbf{W}$ é a matriz diagonal $(\mathrm{n} \times \mathrm{n})$ dos pesos.

Como na regressão linear, outro aspecto de avaliação da qualidade do ajuste é determinar quão influentes são determinadas observações na estimação dos coeficientes de regressão. Suponha que o j-ésimo coeficiente de regressão, quando estimado de um conjunto completo de dados, seja denotado por $\hat{\beta}_{j}$ e o conjunto reduzido de dados, obtido extraindo-se o i-ésimo indivíduo, por $\hat{\beta}_{j}^{(\mathbf{i})}$. A medida de influência da i-ésima observação sobre a estimação de $\beta_{\mathrm{j}}$ é dada por:

$$
\Delta \boldsymbol{\beta}_{\mathbf{j}}^{(\mathbf{i})}=\frac{\hat{\boldsymbol{\beta}}_{\mathrm{j}}-\hat{\boldsymbol{\beta}}_{\mathrm{j}}^{(\mathbf{i})}}{\mathrm{s}\left(\hat{\boldsymbol{\beta}}_{\mathrm{j}}\right)}
$$

onde $s\left(\hat{\beta}_{j}\right)$ é o erro padrão de $\hat{\beta}_{j}$ do conjunto completo de dados. A i-ésima observação terá uma grande influência sobre a estimação de $\beta_{j}$ se $\left|\Delta \beta_{j}^{(i)}\right|$ for grande.

Assim, se algum ponto extremo ou discrepante for detectado, deverá ser verificado se o mesmo poderá ser retirado do conjunto de dados, uma vez que isto pode não ser possível. 


\section{EXEMPLO DE APLICAÇÃO}

Para exemplificar a metodologia de regressão logística, será utilizado o conjunto de dados da Tabela 10 (apêndice), o qual foi fornecido pelo Prof. Ms. Pedro Luiz de Camargo, do Hospital Veterinário da Universidade Estadual de Londrina. Foram coletados dados de 641 cães que deram entrada na hospital com Gastroenterite Hemorrágica, no período de 31/08/93 a 31/08/94.

Como o período de incubação da doença é de 7 dias, as datas para temperaturas e umidade relativa do ar são retroativas à data de entrada do animal no hospital. As variáveis explanatórias estudadas foram:

1. Estações do ano (Est);

21/09 a 20/12 - Primavera - codificada como 0;

21/12 a 20/03 - Verão - codificada como 1 ;

21/03 a 20/06 - Outono - codificada como 2 ;

21/06 a 20/09 - Inverno - codificada como 3;

2. Número de atendimentos (Atend);

Este número se refere ao número de vezes que o animal foi consultado no Hospital Veterinário da Universidade de Londrina;

3. Idade (em meses);

4. Sexo;

Fêmeas - codificada como 0;

Machos - codificado como 1;

5. Diárias;

Este número refere-se ao número de dias que o animal ficou internado; 
6. Temperatura Máxima (Máxima);

7. Temperatura Mínima (Mínima);

8. Amplitude de temperatura (Amplit);

Amplit. $=($ Temp. Máxima $)-($ Temp. Mínima $)$;

9. Umidade relativa do ar (UMID).

Também, procurou-se verificar quais as interações entre as variáveis que pudessem ser significativas. As interações foram codificadas como:

$$
\begin{array}{ll}
\text { estat }=\text { estacões } \times \text { atendimento } & \text { idmin }=\text { idade } \times \text { mínima } \\
\text { estid }=\text { estações } \times \text { idade } & \text { idamp }=\text { idade } \times \text { amplitude } \\
\text { estse }=\text { estações } \times \text { sexo } & \text { idumi }=\text { idade } \times \text { umidade } \\
\text { estdia }=\text { estações } \times \text { diárias } & \text { sexdia }=\text { sexo } \times \text { diárias } \\
\text { estmax }=\text { estações } \times \text { máxima } & \text { sexmax }=\text { sexo } \times \text { máxima } \\
\text { estmin }=\text { estações } \times \text { mínima } & \text { sexmin }=\text { sexo } \times \text { mínima } \\
\text { estamp }=\text { estações } \times \text { amplitude } & \text { sexamp }=\text { sexo } \times \text { amplitude } \\
\text { estumi }=\text { estações } \times \text { umidade } & \text { sexumi }=\text { sexo } \times \text { umidade } \\
\text { atida }=\text { atendimento } \times \text { idade } & \text { diamax }=\text { diárias } \times \text { máxima } \\
\text { atsex }=\text { atendimento } \times \text { sexo } & \text { diamin }=\text { diárias } \times \text { mínima } \\
\text { atdia }=\text { atendimento } \times \text { diárias } & \text { diamp }=\text { diárias } \times \text { amplitude } \\
\text { atmax }=\text { atendimento } \times \text { máxima } & \text { diaumi }=\text { diárias } \times \text { umidade } \\
\text { atmin }=\text { atendimento } \times \text { mínima } & \text { maxumi }=\text { máxima } \times \text { umidade } \\
\text { atamp }=\text { atendimento } \times \text { amplit } & \text { maxmin }=\text { máxima } \times \text { mínima } \\
\text { atumi }=\text { atendimento } \times \text { umidade } & \text { maxamp = máxima } \times \text { amplitude } \\
\text { idsex }=\text { idade } \times \text { sexo } & \text { minamp = mínima } \times \text { amplitude } \\
\text { iddia }=\text { idade } \times \text { diárias } & \text { minumi }=\text { mínima } \times \text { umidade } \\
\text { idmax }=\text { idade } \times \text { máxima } & \text { ampumi }=\text { amplitude } \times \text { umidade }
\end{array}
$$

A variável resposta, óbito, é a condição final do animal após o tratamento, sendo codificada como: $\operatorname{sim}(1)$ ou não (0). O tratamento aplicado foi o mesmo para todos os animais. 
Uma análise preliminar das variáveis foi feita, utilizando o Proc Univariate do SAS (Statistical Analisys System), versão 6.11 para Windows. Os dados foram inicialmente digitados na planilha Excel, versão 7.0 e então lidos pelo SAS, através do comando:

Filename DADOS dde'Excel|Pasta2 !L1C1:L641C11';

sendo que foram obtidas as seguintes informações para as variáveis explanatórias:

Tabela 4 - Média, Erro Padrão da Média, Desvio Padrão e Coeficiente de Variação(\%) das Variáveis Explanatórias.

\begin{tabular}{l:cccc}
\hline & Média & $\begin{array}{c}\text { Erro Padrão } \\
\text { da Média }\end{array}$ & $\begin{array}{c}\text { Desvio } \\
\text { Padrão }\end{array}$ & $\begin{array}{c}\text { Coef. de } \\
\text { Variação (\%) }\end{array}$ \\
\hline Atend & 1,379095 & 0,036243 & 0,917605 & 66,53678 \\
Idade & 7,612168 & 0,461376 & 11,68112 & 153,4532 \\
Diárias & 1,979719 & 0,077888 & 1,971966 & 99,60838 \\
Máxima & 28,47644 & 0,156563 & 3,963863 & 13,91980 \\
Mínima & 16,74883 & 0,143556 & 3,634535 & 21,70023 \\
Amplit & 11,72715 & 0,139042 & 3,52026 & 30,01805 \\
Umidade & 68,38658 & 0,565282 & 14,31179 & 20,92777 \\
\hline
\end{tabular}

Observa-se, pela Tabela 4, uma grande variabilidade dos dados, principalmente, das variáveis idade, diárias e atendimento. Isso se deve ao fato de que as idades dos animais variaram de 1 a 108 meses. O número de diárias variou de algumas horas até 11 dias de internação. Houve casos em que os mesmos animais foram atendidos 9 vezes. A variação da temperatura máxima no período esteve entre $12{ }^{\circ} \mathrm{C}$ e $35,8{ }^{\circ} \mathrm{C}$ enquanto que a variação da temperatura mínima esteve entre $0,1{ }^{\circ} \mathrm{C}$ e $22^{\circ} \mathrm{C}$. A variação da amplitude da temperatura foi de $1.3{ }^{\circ} \mathrm{C}$ a $19,1^{\circ} \mathrm{C}$ e a umidade relativa do ar esteve entre 37,7 e 97,9 .

\section{O programa utilizado para obter os dados acima é:}

Proc univariate; var atend idade diarias máxima mínima amplit umidade; run; 
No estudo dos dados, verificou-se que o modelo mais adequado foi:

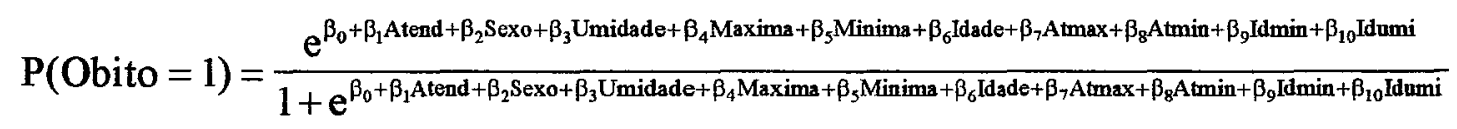

Considerando que os dados são reais e que não houve controle sobre as variáveis, optou-se por utilizar o nível de significância de $\alpha=0,10$ para o modelo. Além das estimativas dos parâmetros, foram obtidos o número de iterações para convergência do modelo, o intervalo de confiança para os parâmetros e para a razão das chances, além da matriz de correlação das variáveis. Para obtenção destes resultados utilizou-se o seguinte programa:

Options nonumber nodate $\mathrm{ps}=1000$;

Filename DADOS dde 'Excel|Pasta2!L1C1:L641C11';

Data DADOS;

Infile DADOS;

Input data \$ estac atend idade sexo diarias obito máxima mínima amplit umidade;

estat $=$ estac $\mathrm{x}$ atend

estid=estac $\mathrm{x}$ idade

estsex=estac $\mathrm{x}$ sexo

estdia $=$ estac $x$ diarias

estmax $=$ estac $\mathrm{x}$ máxima

estmin $=$ estac $\times$ mínima

estamp=estac $x$ amplit

estumi $=$ estac $\times$ umidade

atida $=$ atend $\mathrm{x}$ idade

atsex $=$ atend $x$ sexo

atdia $=$ atend $\mathrm{x}$ diarias

atmax=atend $\mathrm{x}$ máxima

atmin=atend $\mathrm{x}$ mínima idmin=idade $\mathrm{x}$ mínima

idamp=idade $x$ amplit

idumi $=$ idade $x$ umidade

sexdia $=$ sexo $\mathrm{x}$ diarias

sexmax $=$ sexo $x$ máxima

sexmin=sexo $\times$ mínima

sexamp=sexo $x$ amplit

sexumi $=$ sexo $\times$ umidade

diamax=diarias $\mathrm{x}$ máxima

diamin=diarias $\mathrm{x}$ mínima

diamp=diarias $x$ amplit

diaumi $=$ diarias $\times$ umidade

maxumi=máxima $x$ umidade 
atamp $=$ atend $\mathrm{x}$ amplit

atumi $=$ atend $\mathrm{x}$ umidade

idse $\mathrm{x}=$ idade $\mathrm{x}$ sexo

iddia=idade $\mathrm{x}$ diarias

idmax=idade $\mathrm{x}$ máxima maxmin=máxima $\mathrm{x}$ mínima

maxamp=máxima $\mathrm{x}$ amplit

minamp=mínima $x$ amplit

minumi $=$ mínima $\times$ umidade

ampumi $=$ amplit $\mathrm{x}$ umidade

Proc logistic descending;

Model obito=sexo atend umidade máxima idade mínima atmax atmin idmin idumi/ itprint plcl plrl corrb;

Run;

Dos resultados obtidos, tem-se que dos 641 animais, 451 foram ao óbito conforme mostra a Tabela 5:

Tabela 5 - Distribuição dos animais após o tratamento, segundo o des-fecho do estudo.

\begin{tabular}{cc}
\hline Óbito & Total \\
\hline 0 (Não) & 190 \\
1 (Sim) & 451 \\
\hline Total & 641 \\
\hline
\end{tabular}

$\mathrm{Na}$ estimação dos parâmetros do modelo, através da máxima verossimilhança iterativa, foram necessárias 6 iterações para convergência dos parâmetros.

Através do critério da máxima verossimilhança e do método escore podese perceber que o modelo foi significativo ao nível de 0,0790 e 0,0985 , respectivamente.

\begin{tabular}{lccc} 
& \multicolumn{3}{c}{$\begin{array}{c}\text { Intercepto } \\
\mathrm{e}\end{array}$} \\
Critério & $\begin{array}{c}\text { Somente } \\
\text { Intercepto }\end{array}$ & $\begin{array}{c}\text { Covariáveis } \\
\text { Qui-Quadrado para Covariáveis }\end{array}$ \\
-2 LOG L & 779,191 & 762,395 & 16,796 com 10 G.L. $(\mathrm{p}=0,0790)$ \\
Escore &. &. & 16,040 com 10 G.L. $(\mathrm{p}=0,0985)$
\end{tabular}


Tabela 6 - Estimativas de Máxima Verossimilhança, teste de qui-quadrado de Wald e razão de chances.

\begin{tabular}{lccccccc}
\hline \multicolumn{1}{c}{ Variável } & G.L. & $\begin{array}{c}\text { Parâmetro } \\
\text { Estimado }\end{array}$ & $\begin{array}{c}\text { Erro } \\
\text { Padrão }\end{array}$ & $\begin{array}{c}\text { Qui-Quadrado } \\
\text { Wald }\end{array}$ & $\begin{array}{c}\text { Pr }> \\
\text { Qui-Quadrado }\end{array}$ & $\begin{array}{c}\text { Estimativa } \\
\text { Padronizada }\end{array}$ & $\begin{array}{c}\text { Razão } \\
\text { Chances }\end{array}$ \\
\hline INTERCEPTO & 1 & 0,8254 & 1,8214 & 0,2054 & 0,6504 & & \\
SEXO & 1 & 0,4283 & 0,1789 & 5,7290 & $\mathbf{0 , 0 1 6 7}$ & $\mathbf{0 , 1 1 7 6 2 3}$ & 1,535 \\
ATEND & 1 & $-0,1382$ & 1,0366 & 0,0178 & 0,8939 & $-0,069938$ & 0,871 \\
UMIDADE & 1 & $-0,00342$ & 0,0125 & 0,0749 & 0,7844 & $-0,026977$ & 0,997 \\
MAXIMA & 1 & $-0,1442$ & 0,0650 & 4,9279 & $\mathbf{0 , 0 2 6 4}$ & $-0,315153$ & 0,866 \\
DADE & 1 & 0,00770 & 0,0556 & 0,0192 & $\mathbf{0 , 8 8 9 7}$ & $\mathbf{0 , 0 4 9 5 9 5}$ & 1,008 \\
MINIMA & 1 & 0,1423 & 0,0804 & 3,1360 & 0,0766 & 0,285129 & 1,153 \\
ATMAX & 1 & 0,0508 & 0,0343 & 2,1910 & 0,1388 & 0,759356 & 1,052 \\
ATMIN & 1 & $-0,0761$ & 0,0435 & 3,0649 & 0,0800 & $-0,716023$ & 0,927 \\
DMIN & 1 & 0,00610 & 0,00378 & 2,6040 & 0,1066 & 0,694231 & 1,006 \\
DUMI & 1 & $-0,00168$ & 0,00087 & 3,7692 & 0,0522 & $-0,765962$ & 0,998 \\
\hline
\end{tabular}

Como pode ser observado na Tabela 6, o modelo é significativo, ao nível de $\alpha=0,0790$. As variáveis Atend, Umidade, Idade, Atmax, Idmin não têm poder preditivo significativo. Ao se observar a razão das chances, verifica-se que apenas as variáveis sexo e temperatura máxima foram significativas $(\mathrm{p}<0,05)$.

O intervalo de confiança para os parâmetros e para a razão das chances são dados por:

Tabela 7 - Parâmetros Estimados e Intervalos de Confiança a 95\% para as váriáveis explanatórias do modelo de regressão logística.

\begin{tabular}{lccc}
\hline & & \multicolumn{2}{c}{ Limites de Confiança } \\
Variável & Parâmetro Estimado & Inferior & Superior \\
\hline INTERCEPTO & 0,82540 & $-2,73450$ & 4,42680 \\
SEXO & 0,42830 & 0,07970 & 0,78190 \\
ATEND & $-0,13820$ & $-2,27350$ & 1,82420 \\
UMIDADE & $-0,00342$ & $-0,02780$ & 0,02120 \\
MAXIMA & $-0,14420$ & $-0,27450$ & 0,01930 \\
IDADE & 0,00770 & $-0,10660$ & 0,11410 \\
MINIMA & 0,14230 & $-0,01350$ & 0,30220 \\
ATMAX & 0,05080 & $-0,01110$ & 0,12390 \\
ATMIN & $-0,07610$ & $-0,16450$ & 0,00702 \\
IDMIN & 0,00610 & $-0,00052$ & 0,01430 \\
IDUMI & $-0,00168$ & $-0,00351$ & $-0,00010$ \\
\hline
\end{tabular}


Tabela 8 - Razão de Chances Condicional e Intervalo de Confiança de 95\%

\begin{tabular}{lccc}
\hline & & \multicolumn{2}{c}{ Limites de Confiança } \\
\multicolumn{1}{c}{ Variável } & Razão de Chances & Inferior & Superior \\
\hline SEXO & $\mathbf{1 , 5 3 5}$ & 1,083 & 2,186 \\
ATEND & 0,871 & 0,103 & 6,198 \\
UMIDADE & 0,997 & 0,973 & 1,021 \\
MAXIMA & $\mathbf{0 , 8 6 6}$ & 0,760 & 0,981 \\
IDADE & 1,008 & 0,899 & 1,121 \\
MINIMA & 1,153 & 0,987 & 1,353 \\
ATMAX & 1,052 & 0,989 & 1,132 \\
ATMIN & 0,927 & 0,848 & 1,007 \\
IDMIN & 1,006 & 0,999 & 1,014 \\
IDUMI & 0,998 & 0,996 & 1,000 \\
\hline
\end{tabular}

Pela Tabela 8, através da razão de chances, percebe-se que a variável sexo é um fator de risco e variável máxima é um fator de proteção para o óbito dos animais, sendo que a chance de um animal macho vir a morrer é 1,5 vezes a da fềmea, e que o animal estaria protegido em temperaturas máximas não tão elevadas. As demais variáveis, dentre as analisadas, têm a razão de chances próximo a um, indicando que não influenciam na morte do animal.

Tabela 9 - Matriz de correlações estimadas entre as variáveis explanatórias do estudo.

\begin{tabular}{|c|c|c|c|c|c|c|c|c|c|c|}
\hline Variável & INTERCEPTO & SEXO & ATEND & UMIDADE & MAXTMA & IDADE & MINIMA & ATMAX & ATMIN & IDMIN \\
\hline INTERCPTO & 1,0000 & & & & & & & & & \\
\hline SEXO & $-0,0930$ & 1,0000 & & & & & & & & \\
\hline ATEND & $-0,6537$ & 0,0451 & 1,0000 & & & & & & & \\
\hline UMIDADE & $-0,5419$ & 0,0003 & $-0,1022$ & 1,0000 & & & & & & \\
\hline MAXIMA & $-0,7960$ & $-0,0013$ & 0,4525 & 0,4787 & 1,0000 & & & & & \\
\hline IDADE & $-0,1580$ & 0,0186 & $-0,0348$ & 0,1328 & $-0,0548$ & 1,0000 & & & & \\
\hline MINIMA & 0,1146 & 0,0482 & 0,2898 & $-0,5466$ & $-0,6035$ & 0,1859 & 1,0000 & & & \\
\hline ATMAX & 0,5154 & $-0,0130$ & $-0,7253$ & 0,0620 & $-0,7048$ & 0,0374 & 0,2503 & 1,0000 & & \\
\hline ATMIN & 0,2089 & $-0,0450$ & $-0,3998$ & 0,0539 & 0,3135 & $-0,0032$ & $-0,7336$ & $-0,3349$ & 1,0000 & \\
\hline IDMIN & 0,0642 & 0,0350 & $-0,0042$ & 0,2989 & 0,0267 & $-0,5513$ & $-0,2977$ & 0,0307 & $-0,0353$ & 1,0000 \\
\hline IDUMI & 0,0777 & $-0,0568$ & 0,0360 & $-0,4709$ & 0,0136 & $-0,2884$ & 0,1679 & $-0,0702$ & 0,0466 & $-0,6316$ \\
\hline
\end{tabular}


As correlações existentes entre as variáveis, Tabela 9, são muito baixas ou insignificantes. Pode-se afirmar que não há correlação entre as variáveis principais.

Após obterem-se as estimativas dos parâmetros, pode-se verificar se existem pontos que possam influenciar indevidamente o modelo. Esta análise é feita acrescentando-se ao final do programa inicial, as seguintes linhas:

output out=saida $\mathrm{p}=$ predito $\mathrm{l}=$ liminf $\mathrm{u}=$ limsup xbeta=xbeta stdxbeta=erro $\mathrm{h}=\mathrm{hat}$ reschi=respear resdev=resdev;

proc print data $=$ saida;

run;

O resultado da análise de resíduos para os dados se encontram na Tabela 11 (apêndice). Através dos resíduos padronizados de Pearson, determinaram-se os seguintes pontos discrepantes: 9, 73, 166, 189, 247, 277 e 371 .

Os pontos influentes, que são obtidos pela matriz hat, estão assinalados com a cor vermelha, e encontram-se na Tabela 11 (Apêndice).

Assim,

$$
h_{i i}=\frac{2 \times 11}{641}=0,03432,
$$

onde o número 11 corresponde ao número de parâmetros estimados no modelo e o número 641 se refere ao total de animais estudados. Logo, todo ponto com valor acima de $h_{i i}=0,03432$, foi considerado como ponto influente. Os pontos influentes encontrados são relativos às observações: $14,22,30,54,57,58,62,66,79,89,118,122,124,130$, $157,199,200,201,203,215,225,233,268,277,311,315,367,371,404,406,420$, $427,439,444,458,464,498,507,513,515,557,558,559,584,594,604,605,609$ e 618.

Os gráficos dos resíduos podem ser visualizados no Apêndice, gráficos 1 , 2 e 3 . O número de pontos discrepantes é relativamente insignificante, mas não menos 
importante, perante a quantidade de dados. Os pontos discrepantes foram identificados mas não retirados da análise, devido a não identificação da causa.

Como se pôde perceber, as variáveis que mais tiveram influência no fato de o animal vir a falecer foram as variáveis sexo e temperatura máxima; logo, se o animal for macho a chance dele morrer é de 1,5 vezes a chance da fềmea, e também que baixas temperaturas também influenciam no óbito do animal.

A análise das variáveis principais, isoladamente, apontou que a razão de chances da variável sexo foi de 2,5 vezes e que as outras variáveis (estações, atendimentos, idade, diárias, temperatura máxima, temperatura mínima, amplitude de temperatura e umidade) não tinham poder preditivo.

Quando as variáveis foram combinadas, percebeu-se que certas variáveis principais, na presença da variável sexo, passam a ser significativas. Foi o caso de temperatura máxima e temperatura mínima, além das interações entre idade $\mathrm{x}$ umidade, atendimento $\mathrm{x}$ umidade.

$O$ fato de as temperaturas terem efeitos significativos já era esperado. $O$ que não era esperado é que elas só são significativas na presença da variável sexo.

Este experimento, por não ter sido planejado e nem controlado, apresentou alguns problemas. Um deles foi o fato de não se ter uma avaliação da condição do animal no dia da sua entrada no hospital. Há casos em que o animal chega ao hospital em situação muito crítica e outros em que chega em situação melhor, devido à doença ter sido diagnosticada mais rapidamente. Nestes casos, a probabilidade de o animal sobreviver aumenta.

O outro problema, foi o fato de não terem sido testados tipos de tratamentos diferentes para se determinar o melhor procedimento em situações de Gastroenterite Hemorrágica. No presente experimento, usou-se basicamente o mesmo procedimento para todos os animais, que foi o uso de Bactrin, Plasil, Dipilex e Mebendazole.

Quanto ao modelo de regressão logística utilizado, entende-se que foi satisfatório, pois apenas cerca de $10 \%$ dos dados não se ajustaram bem ao modelo. Um 
estudo mais aprofundado deveria ser feito com os pontos influentes, obtidos pela matriz hat, uma vez que existem muitos pontos mascarados pelos dados da primeira análise.

Um estudo com animais doentes e não doentes seria importante para que se pudesse identificar quais os fatores que levam os animais a contraírem a doença. No presente estudo, todos os animais estavam contaminados, e por isso, foi verificado apenas quais as variáveis que podiam agravar o estado geral do animal e levá-lo ao óbito.

Uma maneira de tentar evitar a morte do animal, seria, em temperaturas muito altas ou muito baixas, manter o animal em ambiente com temperatura mais amena e ao primeiro sintoma da doença, levá-lo a um hospital veterinário.

Toda a metodologia utilizada neste trabalho, pode ser aplicada a qualquer tipo de estudo, em animais como eqüinos, bovinos, suínos, etc, desde que a variável resposta em estudo seja dicotômica em um estudo de coorte. 


\section{REFERÊNCIAS BIBLIOGRÁFICAS}

ARANDA-ORDAZ, F. J. On two families os transformations to additivity for binary response data. Biometrika, v.68, n.2, p. 357-363, 1981.

COLLETT, D. Modelling binary data. London: Chapman and Hall, 1991. 369 p.

COPENHAVER, T. W.; MIELKE, P. W. Quantit analysis: a quantal assay refinement. Biometrics, v.33, p. 175-186, 1977.

DIAS, C. T. S. Estatística básica através do sas para Windows. Piracicaba: ESALQ, 1995. $138 \mathrm{p}$

HAUCK JR., W. W.; DONNER, ALLAN Wald's test as applied to hypotheses in "logit" analysis. Journal of the American Statistical Association, v.72, n.360, p. 851-853, 1977.

HOSMER, D. W.; LEMESHOW, S. Applied logistic regression. New York: John Wiley, 1989. $307 \mathrm{p}$.

JENNINGS, D. E. "Outliers" and residual distributions in logistic regression. Journal of the American Statistical Association, v.81, n.396, p. 987-990, 1986. 
KLEINBAUM, D. G. Logistic regression: a self-learning text. New York: SpringerVerlag, 1994. $278 \mathrm{p}$.

MORGAN, B. J. T. The cubic logistic model for quantal assay data Applied Statistical, v.34, n.2, p. 105-113, 1985.

MORGAN, B. J. T. Analysis of quantal response data. London: Chapman \& Hall, 1992. $514 \mathrm{p}$.

PREGIBON, D. Logistic regression diagnostics. The Annals of Statistics, v.9, n.4, p. 705-724, 1981.

PRENTICE, R. L.; PYKE, R. Logistic disease incidence models and case-control studies. Biometrika, v.66, n.3, p. 403-411, 1979.

SOARES, J. F.; COLOSIMO, E. A. Métodos estatísticos na pesquisa clínica. In: REUNIÃO ANUAL DA REGIÃO BRASILEIRA DA SOCIEDADE INTERNACIONAL DE BIOMETRIA, 40; SIMPÓSIO DE ESTATÍSTICA APLICADA À EXPERIMENTAÇÃO AGRONÔMICA, 6., Ribeirão Preto, 1995. Programa e resumos. Jaboticabal, 1995. p. 1-50. 


\section{BIBLIOGRAFIA RECOMENDADA}

AGRESTI, A. Analysis of ordinal categorical data. New York: John Wiley, 1984. $287 \mathrm{p}$.

ASHTON, W. D. The "logit" transformation with special reference to its uses in bioassay. London: Griffin, $1972.88 \mathrm{p}$.

CHATTERJEE, S.; PRICE, B. Regression analysis by example. 2.ed. New York: John Wiley, 1991. $278 \mathrm{p}$.

FINNEY, D. J. Probit analysis. 3.ed. London: Cambridge University Press, 1971. 333 p.

FLEISS, J. L. Statistical methods for rates and proportions. 2.ed. New York: John Wiley, 1981. $321 \mathrm{p}$.

McCULLAGH, P.; NELDER, J. A. Generalized linear models. London: Chapman \& Hall, 1989. $511 \mathrm{p}$.

MIAZAKI, E. S.; STANGENHAUS, G. Métodos para detecção de dados atípicos In: SIMPÓSIO NACIONAL DE PROBABILIDADE E ESTATÍSTICA, 11., Belo Horizonte, 1994. Minicurso. Belo Horizonte: UFMG, 1994. 81 p. 
MONTGOMERY, D. C.; PECK, E. A. Introduction to linear regression analysis. 2.ed. New York: John Wiley, 1992. 527 p.

ROSNER, B. Fundamentals of biostatistics. 4.ed. London: Duxbury Press, 1995. 685 p.

SAS INSTITUTE. Guia do Usuário SAS/STAT: versão 6. Cary, 1996. Cap. 27, p. 1072-1126: The logistic procedure.

SOARES, J. F. Análise estatística de dados epidemiológicos. In: SIMPÓSIO DE ESTATÍSTICA APLICADA À EXPERIMENTAÇÃO AGRONÔMICA, 5., Porto Alegre, 1993. Resumos. Porto Alegre: UFRGS, 1993. 76 p. 
A P Ê N D I C E 
Tabela 10: Dados Coletados dos Animais no Período de 31/08/93 a 31/08/94.

\begin{tabular}{|c|c|c|c|c|c|c|c|c|c|c|}
\hline Data & Estações & Atendim. & Idade & Sexo & Diárias & óbito & $\begin{array}{l}\text { Temper. } \\
\text { Máxima }\end{array}$ & $\begin{array}{l}\text { Temper. } \\
\text { Mínima }\end{array}$ & $\begin{array}{l}\text { Alplitude } \\
\text { Tenper. }\end{array}$ & Umidade \\
\hline $31 / 08 / 93$ & 3 & 2 & 2 & 1 & 3 & 0 & 20,9 & 14,2 & 6,7 & 89,2 \\
\hline $01 / 09 / 93$ & 3 & 2 & 4 & 0 & 7 & $\mathbf{0}$ & 25,0 & 13,8 & 11,2 & 78,1 \\
\hline $01 / 09 / 93$ & 3 & 1 & 4 & 1 & 2 & 1 & 25,0 & 13,8 & 11,2 & 78,1 \\
\hline $02 / 09 / 93$ & 3 & 1 & 15 & 0 & 2 & 0 & 29,1 & 12,5 & 16,6 & 57,9 \\
\hline $02 / 09 / 93$ & 3 & 2 & 12 & 0 & 4 & 0 & 29,1 & 12,5 & 16,6 & 57,9 \\
\hline $02 / 09 / 93$ & 3 & 1 & 7 & 0 & 2 & 0 & 29,1 & 12,5 & 16,6 & 57,9 \\
\hline $02 / 09 / 93$ & 3 & 1 & 3 & 1 & 4 & 0 & 29,1 & 12,5 & 16,6 & 57,9 \\
\hline $02 / 09 / 93$ & 3 & 1 & 4 & 1 & 0 & 0 & 29,1 & 12,5 & 16,6 & 57,9 \\
\hline $02 / 09 / 93$ & 3 & 1 & 4 & 0 & 4 & 1 & 29,1 & 12,5 & 16,6 & 57,9 \\
\hline $03 / 09 / 93$ & 3 & 1 & 5 & 0 & 0 & 0 & 31,1 & 14,7 & 16,4 & 54,4 \\
\hline $03 / 09 / 93$ & 3 & 3 & 4 & 1 & 3 & 0 & 31,1 & 14,7 & 16,4 & 54,4 \\
\hline $04 / 09 / 93$ & 3 & 1 & 4 & 1 & o & 0 & 30,1 & 15,8 & 14,3 & 52,6 \\
\hline $04 / 09 / 93$ & 3 & 2 & 4 & 0 & 1 & 1 & 30,1 & 15,8 & 14,3 & 52,6 \\
\hline $05 / 09 / 93$ & 3 & 4 & 7 & 1 & 3 & 1 & 32,3 & 15,6 & 16,7 & 42,2 \\
\hline $06 / 09 / 93$ & 3 & 2 & 4 & 0 & 3 & 0 & 32,8 & 17,4 & 15,4 & 45,7 \\
\hline $06 / 09 / 93$ & 3 & 2 & 12 & 1 & 2 & 1 & 32,8 & 17,4 & 15,4 & 45,7 \\
\hline $06 / 09 / 93$ & 3 & 1 & 3 & 1 & 1 & 1 & 32,8 & 17,4 & 15,4 & 45,7 \\
\hline $07 / 09 / 93$ & 3 & 1 & 3 & 0 & 6 & 0 & 32,2 & 20,1 & 12,1 & 62,8 \\
\hline $07 / 09 / 93$ & 3 & 1 & 5 & 1 & 2 & 1 & 32,2 & 20,1 & 12,1 & 62,8 \\
\hline $08 / 09 / 93$ & 3 & 1 & 3 & 1 & 0 & 0 & 28,9 & 14,4 & 14,5 & 70,0 \\
\hline $08 / 09 / 93$ & 3 & 1 & 3 & 1 & 0 & 0 & 28,9 & 14,4 & 14,5 & 70,0 \\
\hline $08 / 09 / 93$ & 3 & 4 & 5 & 0 & 1 & 1 & 28,9 & 14,4 & 14,5 & 70,0 \\
\hline $09 / 09 / 93$ & 3 & 1 & 5 & 0 & 0 & 0 & 30,4 & 16,0 & 14,4 & 62,6 \\
\hline $09 / 09 / 93$ & 3 & i & 24 & 0 & 2 & 0 & 30,4 & 16,0 & 14,4 & 62,6 \\
\hline $09 / 09 / 93$ & 3 & 1 & 30 & 0 & 2 & 1 & 30,4 & 16,0 & 14,4 & 62,6 \\
\hline $10 / 09 / 93$ & 3 & 2 & 4 & 0 & 4 & o & 26,7 & 18,3 & 8,4 & 70,2 \\
\hline $10 / 09 / 93$ & 3 & 1 & 4 & 1 & 4 & 0 & 26,7 & 18,3 & 8,4 & 70,2 \\
\hline $10 / 09 / 93$ & 3 & 1 & 12 & 1 & 1 & 0 & 26,7 & 18,3 & 8,4 & 70,2 \\
\hline $11 / 09 / 93$ & 3 & 1 & 3 & 0 & 2 & 0 & 26,6 & 11,4 & 15,2 & 71,0 \\
\hline $11 / 09 / 93$ & 3 & 3 & 3 & 0 & 3 & 1 & 26,6 & 11,4 & 15,2 & 71,0 \\
\hline $11 / 09 / 93$ & 3 & 1 & 3 & 1 & 2 & 1 & 26,6 & 11,4 & 15,2 & 71,0 \\
\hline $12 / 09 / 93$ & 3 & 1 & 12 & 0 & 4 & 0 & 31,4 & 14,0 & 17,4 & 61,9 \\
\hline $12 / 09 / 93$ & 3 & 2 & 2 & 1 & 3 & o & 31,4 & 14,0 & 17,4 & 61,9 \\
\hline $12 / 09 / 93$ & 3 & 2 & 8 & 1 & 5 & 1 & 31,4 & 14,0 & 17,4 & 61,9 \\
\hline $13 / 09 / 93$ & 3 & 1 & 3 & 0 & 1 & 0 & 34,1 & 17,1 & 17,0 & 47,4 \\
\hline $13 / 09 / 93$ & 3 & 1 & 2 & 1 & 1 & $\mathbf{0}$ & 34,1 & 17,1 & 17,0 & 47,4 \\
\hline $13 / 09 / 93$ & 3 & 3 & 12 & 0 & 5 & 1 & 34,1 & 17,1 & 17,0 & 47,4 \\
\hline $14 / 09 / 93$ & 3 & 1 & 3 & 0 & 2 & 0 & 31,8 & 19,2 & 12,6 & 69,2 \\
\hline $14 / 09 / 93$ & 3 & 1 & 2 & 1 & 0 & 0 & 31,8 & 19,2 & 12,6 & 69,2 \\
\hline $15 / 09 / 93$ & 3 & 1 & 5 & 1 & 0 & 0 & 23,0 & 18,1 & 4,9 & 85,4 \\
\hline $15 / 09 / 93$ & 3 & 1 & 5 & 1 & 3 & 0 & 23,0 & 18,1 & 4,9 & 85,4 \\
\hline $15 / 09 / 93$ & 3 & 1 & 6 & 1 & 1 & 0 & 23,0 & 18,1 & 4,9 & 85,4 \\
\hline
\end{tabular}

(continua...) 
(continuação...)

Tabela 10: Dados Coletados dos Animais no Período de 31/08/93 a 31/08/94.

\begin{tabular}{|c|c|c|c|c|c|c|c|c|c|c|}
\hline Data & Estaçōes & Atendim. & Idade & Sexo & Diárias & óbito & $\begin{array}{l}\text { Temper. } \\
\text { Máxima }\end{array}$ & $\begin{array}{l}\text { Teaper. } \\
\text { Mínina }\end{array}$ & $\begin{array}{l}\text { Anplitude } \\
\text { Tenper. }\end{array}$ & Umidade \\
\hline $16 / 09 / 93$ & 3 & 2 & 3 & 1 & 2 & 0 & 21,6 & 18,4 & 3,2 & 96,4 \\
\hline $16 / 09 / 93$ & 3 & 1 & 3 & 1 & 3 & 0 & 21,6 & 18,4 & 3,2 & 96,4 \\
\hline $16 / 09 / 93$ & 3 & 2 & 3 & 1 & 2 & 1 & 21,6 & 18,4 & 3,2 & 96,4 \\
\hline $17 / 09 / 93$ & 3 & 1 & 3 & 0 & 4 & 0 & 22,8 & 17,8 & 5,0 & 92,5 \\
\hline $18 / 09 / 93$ & 3 & 1 & 3 & 1 & 2 & 0 & 22,9 & 14,9 & 8,0 & 75,8 \\
\hline $18 / 09 / 93$ & 3 & 1 & 4 & 1 & 1 & 0 & 22,9 & 14,9 & 8,0 & 75,8 \\
\hline $18 / 09 / 93$ & 3 & 1 & 12 & 1 & 3 & 0 & 22,9 & 14,9 & 8,0 & 75,8 \\
\hline $19 / 09 / 93$ & 3 & 1 & 3 & 1 & 3 & 0 & 22,6 & 10,6 & 12,0 & 63,5 \\
\hline $19 / 09 / 93$ & 3 & 1 & 4 & 1 & 0 & 0 & 22,6 & 10,6 & 12,0 & 63,5 \\
\hline $19 / 09 / 93$ & 3 & 1 & 4 & 1 & 1 & 1 & 22,6 & 10,6 & 12,0 & 63,5 \\
\hline $20 / 09 / 93$ & 3 & 1 & 6 & 0 & 3 & 0 & 23,2 & 11,7 & 11,5 & 66,6 \\
\hline $20 / 09 / 93$ & 3 & 3 & 7 & 1 & 3 & 0 & 23,2 & 11,7 & $\$ 1,5$ & 66,6 \\
\hline $20 / 09 / 93$ & 3 & 1 & 4 & 1 & 0 & 0 & 23,2 & 11,7 & 11,5 & 66,6 \\
\hline $21 / 09 / 93$ & 0 & 1 & 4 & 1 & 0 & 0 & 18,2 & 13,0 & 5,2 & 96,1 \\
\hline $21 / 09 / 93$ & 0 & 3 & 4 & 1 & 2 & 0 & 18,2 & 13,0 & 5,2 & 96,1 \\
\hline $21 / 09 / 93$ & 0 & 1 & 24 & 1 & 0 & 0 & 18,2 & 13,0 & 5,2 & 96,1 \\
\hline $21 / 09 / 93$ & 0 & 2 & 5 & 0 & 1 & 1 & 18,2 & 13,0 & 5,2 & 96,1 \\
\hline $22 / 09 / 93$ & 0 & 1 & 10 & 1 & 4 & $\mathbf{0}$ & 23,4 & 14,2 & 9,2 & 77,5 \\
\hline $22 / 09 / 93$ & 0 & 1 & 5 & 1 & 1 & 1 & 23,4 & 14,2 & 9,2 & 77,5 \\
\hline $23 / 09 / 93$ & 0 & 2 & 108 & 0 & 3 & 0 & 26,9 & 12,2 & 14,7 & 59,7 \\
\hline $23 / 09 / 93$ & 0 & 1 & 6 & 1 & 3 & 0 & 26,9 & 12,2 & 14,7 & 59,7 \\
\hline $23 / 09 / 93$ & 0 & 1 & 6 & 1 & 1 & 1 & 26,9 & 12,2 & 14,7 & 59,7 \\
\hline $23 / 09 / 93$ & 0 & 1 & 2,3 & 1 & 1 & 1 & 26,9 & 12,2 & 14,7 & 59,7 \\
\hline $24 / 09 / 93$ & 0 & 3 & 46 & 1 & 2 & 0 & 29,4 & 13,1 & 16,3 & 56,1 \\
\hline $25 / 09 / 93$ & 0 & 1 & 1,3 & 1 & 4 & 1 & 27,6 & 15,4 & 12,2 & 60,1 \\
\hline $26 / 09 / 93$ & 0 & 1 & 6 & 1 & 3 & 0 & 24,3 & 18,3 & 6,0 & 86,5 \\
\hline $27 / 09 / 93$ & 0 & 1 & 4 & 0 & 4 & 0 & 33,5 & 16,5 & 17,0 & 58,1 \\
\hline $27 / 09 / 93$ & 0 & 2 & 1,3 & 0 & 0 & 0 & 33,5 & 16,5 & 17,0 & 58,1 \\
\hline $27 / 09 / 93$ & 0 & 1 & 4 & 1 & 0 & 0 & 33,5 & 16,5 & 17,0 & 58,1 \\
\hline $27 / 09 / 93$ & 0 & 1 & 5 & 1 & 3 & 0 & 33,5 & 16,5 & 17,0 & 58,1 \\
\hline $27 / 09 / 93$ & 0 & 1 & 8 & 0 & 1 & 1 & 33,5 & 16,5 & 17,0 & 58,1 \\
\hline $27 / 09 / 93$ & 0 & 1 & 3 & 1 & 1 & 1 & 33,5 & 16,5 & 17,0 & 58,1 \\
\hline $27 / 09 / 93$ & 0 & 3 & 4 & 1 & 9 & 1 & 33,5 & 16,5 & 17,0 & 58,1 \\
\hline $27 / 09 / 93$ & 0 & 1 & 42 & 1 & 1 & 1 & 33,5 & 16,5 & 17,0 & 58,1 \\
\hline $28 / 09 / 93$ & 0 & 1 & 5 & 0 & 0 & 0 & 27,2 & 19,1 & 8,1 & 94,3 \\
\hline $28 / 09 / 93$ & 0 & 1 & 7 & 1 & 0 & 0 & 27,2 & 19,1 & 8,1 & 94,3 \\
\hline $29 / 09 / 93$ & 0 & 3 & 4 & 0 & 3 & 0 & 19,9 & 15,0 & 4,9 & 92,9 \\
\hline $29 / 09 / 93$ & 0 & 2 & 6 & 0 & 3 & 0 & 19,9 & 15,0 & 4,9 & 92,9 \\
\hline $29 / 09 / 93$ & 0 & 2 & 2 & 1 & 7 & 1 & 19,9 & 15,0 & 4,9 & 92,9 \\
\hline
\end{tabular}


(continuação...)

Tabela 10: Dados Coletados dos Animais no Período de 31/08/93 a 31/08/94.

\begin{tabular}{|c|c|c|c|c|c|c|c|c|c|c|}
\hline Data & Estações & Atendim. & Idade & sexo & Diárias & óbito & $\begin{array}{l}\text { Temper. } \\
\text { Máxima }\end{array}$ & $\begin{array}{l}\text { Temper. } \\
\text { Mínima }\end{array}$ & $\begin{array}{l}\text { Amplitude } \\
\text { Temper. }\end{array}$ & Umidade \\
\hline $29 / 09 / 93$ & 0 & 1 & 3 & 1 & 1 & 1 & 19,9 & 15,0 & 4,9 & 92,9 \\
\hline $30 / 09 / 93$ & 0 & 1 & 4 & 0 & 0 & 0 & 17,4 & 16,1 & 1,3 & 96,6 \\
\hline $30 / 09 / 93$ & 0 & 1 & 2 & 0 & 4 & 1 & 17,4 & 16,1 & 1,3 & 96,6 \\
\hline $30 / 09 / 93$ & 0 & 2 & 2 & 0 & 5 & 1 & 17,4 & 16,1 & 1,3 & 96,6 \\
\hline $30 / 09 / 93$ & 0 & 1 & 2 & 1 & 5 & 1 & 17,4 & 16,1 & 1,3 & 96,6 \\
\hline $01 / 10 / 93$ & 0 & 2 & 6 & 0 & 0 & 0 & 19,7 & 15,4 & 4,3 & 96,2 \\
\hline $01 / 10 / 93$ & 0 & 1 & 5 & 1 & 5 & 0 & 19,7 & 15,4 & 4,3 & 96,2 \\
\hline $01 / 10 / 93$ & 0 & 1 & 18 & 1 & 4 & 1 & 19,7 & 15,4 & 4,3 & 96,2 \\
\hline $04 / 10 / 93$ & 0 & 2 & 6 & 0 & 3 & 0 & 23,4 & 15,4 & 8,0 & 66,2 \\
\hline $04 / 10 / 93$ & 0 & 1 & 4 & 1 & 5 & $\mathbf{0}$ & 23,4 & 15,4 & 8,0 & 66,2 \\
\hline $04 / 10 / 93$ & 0 & 1 & 2,5 & 1 & 3 & 0 & 23,4 & 15,4 & 8,0 & 66,2 \\
\hline $04 / 10 / 93$ & 0 & 1 & 6 & 0 & 2 & 1 & 23,4 & 15,4 & 8,0 & 66,2 \\
\hline $06 / 10 / 93$ & 0 & 2 & 4 & 0 & 2 & 0 & 28,6 & 14,8 & 13,8 & 65,8 \\
\hline $06 / 10 / 93$ & 0 & 1 & 15 & 1 & 5 & 0 & 28,6 & 14,8 & 13,8 & 65,8 \\
\hline $07 / 10 / 93$ & 0 & 3 & 3 & 0 & 4 & 0 & 25,3 & 16,7 & 8,6 & 81,5 \\
\hline $07 / 10 / 93$ & 0 & 2 & 9 & 1 & 6 & 0 & 25,3 & 16,7 & 8,6 & 81,5 \\
\hline $08 / 10 / 93$ & 0 & 1 & 3 & 0 & 7 & $\mathbf{0}$ & 20,8 & 16,6 & 4,2 & 97,4 \\
\hline $09 / 10 / 93$ & 0 & 1 & 20 & 0 & 6 & 0 & 24,4 & 17,2 & 7,2 & 86,1 \\
\hline $10 / 10 / 93$ & 0 & 1 & 8 & 0 & 1 & 0 & 27,7 & 17,4 & 10,3 & 77,6 \\
\hline $10 / 10 / 93$ & 0 & 1 & 6 & 1 & 4 & 0 & 27,7 & 17,4 & 10,3 & 77,6 \\
\hline $10 / 10 / 93$ & 0 & 1 & 4 & 1 & 1 & 1 & 27,7 & 17,4 & 10,3 & 77,6 \\
\hline $11 / 10 / 93$ & 0 & 2 & 4 & 0 & 1 & 0 & 25,1 & 19,6 & 5,5 & 91,7 \\
\hline $13 / 10 / 93$ & 0 & 1 & 4 & 0 & 1 & 0 & 24,4 & 14,4 & 10,0 & 64,6 \\
\hline $13 / 10 / 93$ & 0 & 1 & 5 & 1 & 3 & 0 & 24,4 & 14,4 & 10,0 & 64,6 \\
\hline $14 / 10 / 93$ & 0 & 1 & 6 & 0 & 4 & 0 & 23,8 & 10,3 & 13,5 & 54,1 \\
\hline $14 / 10 / 93$ & 0 & 1 & 4 & 0 & 1 & 1 & 23,8 & 10,3 & 13,5 & 54,1 \\
\hline $15 / 10 / 93$ & 0 & 1 & 4 & 1 & 6 & 0 & 27,2 & 12,0 & 15,2 & 55,5 \\
\hline $16 / 10 / 93$ & 0 & 1 & 1 & 0 & 0 & 0 & 30,3 & 14,0 & 16,3 & 53,4 \\
\hline $16 / 10 / 93$ & 0 & 1 & 5 & 1 & 2 & 0 & 30,3 & 14,0 & 16,3 & 53,4 \\
\hline $16 / 10 / 93$ & 0 & 1 & 3 & 1 & 0 & 0 & 30,3 & 14,0 & 16,3 & 53,4 \\
\hline $17 / 10 / 93$ & 0 & 1 & 5 & $t$ & 2 & 0 & 32,5 & 17,8 & 14,7 & 49,2 \\
\hline $18 / 10 / 93$ & 0 & 1 & 4 & 0 & 0 & 0 & 33,4 & 18,3 & 15,1 & 46,3 \\
\hline $18 / 10 / 93$ & 0 & 1 & 4 & 0 & 2 & 0 & 33,4 & 18,3 & 15,1 & 46,3 \\
\hline $18 / 10 / 93$ & 0 & 1 & 2 & 1 & 0 & 0 & 33,4 & 18,3 & 15,1 & 46,3 \\
\hline $18 / 10 / 93$ & 0 & 2 & 16 & 0 & 0 & 1 & 33,4 & 18,3 & 15,1 & 46,3 \\
\hline $19 / 10 / 93$ & 0 & 2 & 5 & 0 & 0 & 0 & 33,9 & 19,3 & 14,6 & 44,3 \\
\hline $19 / 10 / 93$ & 0 & 1 & 36 & 1 & 1 & 0 & 33,9 & 19,3 & 14,6 & 44,3 \\
\hline $19 / 10 / 93$ & 0 & 1 & 5 & 1 & 3 & 0 & 33,9 & 19,3 & 14,6 & 44,3 \\
\hline $19 / 10 / 93$ & 0 & 1 & 5 & 0 & 4 & 1 & 33,9 & 19,3 & 14,6 & 44,3 \\
\hline
\end{tabular}


(continuação...)

Tabela 10: Dados Coletados dos Animais no Período de 31/08/93 a 31/08/94.

\begin{tabular}{|c|c|c|c|c|c|c|c|c|c|c|}
\hline Data & Estaçōes & Atendim. & Idade & Sexo & Diárias & óbito & $\begin{array}{l}\text { Temper. } \\
\text { Máxima }\end{array}$ & $\begin{array}{l}\text { Temper. } \\
\text { Mínima }\end{array}$ & $\begin{array}{l}\text { Amplitude } \\
\text { Tenper. }\end{array}$ & Unidade \\
\hline $20 / 10 / 93$ & 0 & 2 & 11 & 0 & 0 & 0 & 35,6 & 18,7 & 16,9 & 50,0 \\
\hline $21 / 10 / 93$ & 0 & 1 & 29 & 0 & 0 & 0 & 30,6 & 21,0 & 9,6 & 60,9 \\
\hline $21 / 10 / 93$ & 0 & 1 & 5 & 1 & 7 & 0 & 30,6 & 21,0 & 9,6 & 60,9 \\
\hline $21 / 10 / 93$ & 0 & 4 & 15 & 1 & 0 & 0 & 30,6 & 21,0 & 9,6 & 60,9 \\
\hline $21 / 10 / 93$ & 0 & 1 & 5 & 0 & 2 & 1 & 30,6 & 21,0 & 9,6 & 60,9 \\
\hline $21 / 10 / 93$ & 0 & 1 & 1 & 0 & 1 & 1 & 30,6 & 21,0 & 9,6 & 60,9 \\
\hline $21 / 10 / 93$ & 0 & 1 & 3 & 1 & 1 & 1 & 30,6 & 21,0 & 9,6 & 60,9 \\
\hline $22 / 10 / 93$ & 0 & 2 & 6 & 1 & 2 & 0 & 31,1 & 19,0 & 12,1 & 57,9 \\
\hline $23 / 10 / 93$ & 0 & 1 & 12 & 0 & 1 & 1 & 31,1 & 16,4 & 14,7 & 52,0 \\
\hline $23 / 10 / 93$ & 0 & 1 & 48 & 0 & 2 & 1 & 31,1 & 16,4 & 14,7 & 52,0 \\
\hline $24 / 10 / 93$ & 0 & $t$ & 1 & 0 & 0 & 0 & 33,7 & 18,6 & 15,1 & 54,5 \\
\hline $25 / 10 / 93$ & 0 & 1 & 4 & 0 & 4 & 0 & 29,4 & 19,7 & 9,7 & 61,9 \\
\hline $25 / 10 / 93$ & 0 & 2 & 2 & 0 & 0 & 0 & 29,4 & 19,7 & 9,7 & 61,9 \\
\hline $25 / 10 / 93$ & 0 & 1 & 14 & 0 & 0 & 0 & 29,4 & 19,7 & 9,7 & 61,9 \\
\hline $25 / 10 / 93$ & 0 & 2 & 2 & 0 & 1 & 0 & 29,4 & 19,7 & 9,7 & 61,9 \\
\hline $25 / 10 / 93$ & 0 & 1 & 12 & 0 & 0 & 0 & 29,4 & 19,7 & 9,7 & 61,9 \\
\hline $25 / 10 / 93$ & 0 & 2 & 2 & 1 & 2 & 1 & 29,4 & 19,7 & 9,7 & 61,9 \\
\hline $26 / 10 / 93$ & 0 & 2 & 2 & 1 & 9 & 1 & 25,2 & 18,8 & 6,4 & 86,8 \\
\hline $27 / 10 / 93$ & 0 & 1 & 4 & 0 & 0 & 0 & 30,4 & 19,2 & 11,2 & 67,7 \\
\hline $27 / 10 / 93$ & 0 & 1 & 3 & 0 & 2 & 0 & 30,4 & 19,2 & 11,2 & 67,7 \\
\hline $27 / 10 / 93$ & 0 & 2 & 4 & 0 & 7 & 0 & 30,4 & 19,2 & 11,2 & 67,7 \\
\hline $27 / 10 / 93$ & 0 & 2 & 4 & 0 & 7 & 0 & 30,4 & 19,2 & 11,2 & 67,7 \\
\hline $27 / 10 / 93$ & 0 & 2 & 2,3 & 1 & 0 & 0 & 30,4 & 19,2 & 11,2 & 67,7 \\
\hline $27 / 10 / 93$ & 0 & 1 & 3 & 1 & 5 & 1 & 30,4 & 19,2 & 11,2 & 67,7 \\
\hline $27 / 10 / 93$ & 0 & 1 & 3 & 1 & 2 & 1 & 30,4 & 19,2 & 11,2 & 67,7 \\
\hline $28 / 10 / 93$ & 0 & 1 & 12 & 1 & 2 & 1 & 32,0 & 18,8 & 13,2 & 73,6 \\
\hline $29 / 10 / 93$ & 0 & 1 & 17 & 0 & 1 & 0 & 33,1 & 20,9 & 12,2 & 72,6 \\
\hline $29 / 10 / 93$ & 0 & 2 & 7 & 0 & 6 & 0 & 33,1 & 20,9 & 12,2 & 72,6 \\
\hline $30 / 10 / 93$ & 0 & 1 & 3 & 0 & 0 & 0 & 24,4 & 19,4 & 5,0 & 85,4 \\
\hline $30 / 10 / 93$ & 0 & 1 & 5 & 0 & 2 & 1 & 24,4 & 19,4 & 5,0 & 85,4 \\
\hline $31 / 10 / 93$ & 0 & 1 & 2,7 & 0 & 4 & 0 & 27,6 & 19,0 & 8,6 & 80,9 \\
\hline $31 / 10 / 93$ & 0 & 1 & 3 & 0 & 6 & 1 & 27,6 & 19,0 & 8,6 & 80,9 \\
\hline $01 / 11 / 93$ & 0 & 1 & 5 & 0 & 3 & 0 & 27,0 & 16,4 & 10,6 & 79,5 \\
\hline $01 / 11 / 93$ & 0 & 1 & 12 & 1 & 4 & 0 & 27,0 & 16,4 & 10,6 & 79,5 \\
\hline $01 / 11 / 93$ & 0 & 1 & 6 & 0 & 1 & 1 & 27,0 & 16,4 & 10,6 & 79,5 \\
\hline $02 / 11 / 93$ & 0 & 1 & 2,1 & 0 & 1 & 0 & 30,6 & 21,0 & 9,6 & 71,6 \\
\hline $02 / 11 / 93$ & 0 & 1 & 66 & 0 & 1 & 1 & 30,6 & 21,0 & 9,6 & 71,6 \\
\hline $03 / 11 / 93$ & 0 & 1 & 4 & 1 & 2 & 0 & 27,9 & 16,8 & 11,1 & 62,5 \\
\hline $03 / 11 / 93$ & 0 & 1 & 6 & 1 & 1 & 1 & 27,9 & 16,8 & 11,1 & 62,5 \\
\hline
\end{tabular}


(continuação...)

Tabela 10: Dados Coletados dos Animais no Período de 31/08/93 a 31/08/94.

\begin{tabular}{|c|c|c|c|c|c|c|c|c|c|c|}
\hline Data & Estaçōes & Atendim. & Idade & Sexo & Diárias & óbito & $\begin{array}{l}\text { Tewper. } \\
\text { Máxima }\end{array}$ & $\begin{array}{l}\text { Temper. } \\
\text { Mínima }\end{array}$ & $\begin{array}{l}\text { Amplitude } \\
\text { Temper. }\end{array}$ & Umidade \\
\hline $04 / 11 / 93$ & 0 & 1 & 6 & 0 & 1 & 0 & 28,0 & 14,8 & 13,2 & 65,1 \\
\hline $04 / 11 / 93$ & 0 & 3 & 4 & 0 & 3 & 0 & 28,0 & 14,8 & 13,2 & 65,1 \\
\hline $04 / 11 / 93$ & 0 & 1 & 8 & 0 & 0 & $\mathbf{0}$ & 28,0 & 14,8 & 13,2 & 65,1 \\
\hline $04 / 11 / 93$ & 0 & 2 & 2 & 1 & 0 & 0 & 28,0 & 14,8 & 13,2 & 65,1 \\
\hline $04 / 1 t / 93$ & 0 & 1 & 3 & 1 & 8 & 0 & 28,0 & 14,8 & 13,2 & 65,1 \\
\hline $04 / 11 / 93$ & 0 & 1 & 3 & 1 & 1 & 0 & 28,0 & 14,8 & 13,2 & 65,1 \\
\hline $05 / 11 / 93$ & 0 & 1 & 5 & 0 & 3 & 1 & 30,6 & 14,9 & 15,7 & 60,3 \\
\hline $06 / 11 / 93$ & 0 & 1 & 3 & 0 & 0 & 0 & 33,8 & 19,9 & 13,9 & 55,9 \\
\hline $06 / 11 / 93$ & 0 & 1 & 4 & 1 & 0 & 0 & 33,8 & 19,9 & 13,9 & 55,9 \\
\hline $06 / 11 / 93$ & 0 & 1 & 3 & 1 & 0 & 0 & 33,8 & 19,9 & 13,9 & 55,9 \\
\hline $07 / 11 / 93$ & 0 & 1 & 5 & 0 & 3 & 0 & 34,6 & 20,8 & 13,8 & 44,8 \\
\hline $07 / 11 / 93$ & 0 & 1 & 8 & 1 & 2 & 1 & 34,6 & 20,8 & 13,8 & 44,8 \\
\hline $08 / 11 / 93$ & 0 & 2 & 2 & 0 & 5 & 0 & 32,2 & 21,0 & 11,2 & 68,6 \\
\hline $08 / 11 / 93$ & $\mathbf{0}$ & 1 & 4 & 1 & o & 0 & 32,2 & 21,0 & 11,2 & 68,6 \\
\hline $08 / 11 / 93$ & 0 & 2 & 8 & 1 & 0 & 0 & 32,2 & 21,0 & 11,2 & 68,6 \\
\hline $08 / 11 / 93$ & 0 & 1 & 6 & 1 & 1 & 1 & 32,2 & 21,0 & 11,2 & 68,6 \\
\hline $09 / 11 / 93$ & 0 & 3 & 6 & 0 & 4 & o & 31,8 & 17,7 & 14,1 & 55,1 \\
\hline $09 / 11 / 93$ & 0 & 1 & 8 & 0 & 0 & 0 & 31,8 & 17,7 & 14,1 & 55,1 \\
\hline $09 / 11 / 93$ & 0 & 1 & 8 & 1 & 0 & 0 & 31,8 & 17,7 & 14,1 & 55,1 \\
\hline $09 / 11 / 93$ & 0 & 1 & 12 & 1 & 0 & 0 & 31,8 & 17,7 & 14,1 & 55,1 \\
\hline $09 / 11 / 93$ & 0 & 1 & 1 & 1 & 0 & 0 & 31,8 & 17,7 & 14,1 & 55,1 \\
\hline $09 / 11 / 93$ & 0 & 1 & 6 & 0 & 1 & 1 & 31,8 & 17,7 & 14,1 & 55,1 \\
\hline $09 / 11 / 93$ & 0 & 1 & 3 & 1 & 3 & 1 & 31,8 & 17,7 & $14, t$ & 55,1 \\
\hline $10 / 11 / 93$ & 0 & 1 & 7 & 0 & 3 & 1 & 31,2 & 17,7 & 13,5 & 63,0 \\
\hline $10 / 11 / 93$ & 0 & 1 & 4 & 1 & 3 & 1 & 31,2 & 17,7 & 13,5 & 63,0 \\
\hline $10 / 11 / 93$ & 0 & 1 & 3 & 1 & 1 & 1 & 31,2 & 17,7 & 13,5 & 63,0 \\
\hline $11 / 11 / 93$ & 0 & 1 & 6 & 1 & 0 & 0 & 31,6 & 17,4 & 14,2 & 38,3 \\
\hline $11 / 11 / 93$ & 0 & 1 & 5 & 1 & 0 & 0 & 31,6 & 17,4 & 14,2 & 38,3 \\
\hline $12 / 11 / 93$ & 0 & 1 & 12 & 1 & 0 & 0 & 33,7 & 16,3 & 17,4 & 46,9 \\
\hline $12 / 11 / 93$ & 0 & 1 & 4 & 0 & 2 & 1 & 33,7 & 16,3 & 17,4 & 46,9 \\
\hline $13 / 11 / 93$ & 0 & 2 & 2 & 1 & 5 & 0 & 27,0 & 19,5 & 7,5 & 89,8 \\
\hline $13 / 11 / 93$ & 0 & 1 & 4 & 0 & 4 & 1 & 27,0 & 19,5 & 7,5 & 89,8 \\
\hline $13 / 11 / 93$ & 0 & 1 & 2 & 1 & 5 & 1 & 27,0 & 19,5 & 7,5 & 89,8 \\
\hline $15 / 11 / 93$ & 0 & 2 & 6 & 0 & 3 & 0 & 30,2 & 13,3 & 16,9 & 59,8 \\
\hline $15 / 11 / 93$ & 0 & 1 & 5 & 0 & 1 & 0 & 30,2 & 13,3 & 16,9 & 59,8 \\
\hline $15 / 11 / 93$ & 0 & 1 & 5 & 1 & 5 & 0 & 30,2 & 13,3 & 16,9 & 59,8 \\
\hline $15 / 11 / 93$ & 0 & 1 & 6 & 1 & 0 & 0 & 30,2 & 13,3 & 16,9 & 59,8 \\
\hline $16 / 11 / 93$ & 0 & 1 & 3 & 0 & 2 & 0 & 31,8 & 15,9 & 15,9 & 55,1 \\
\hline $16 / 11 / 93$ & 0 & 1 & 4 & 1 & 3 & 0 & 31,8 & 15,9 & 15,9 & 55,1 \\
\hline
\end{tabular}

(continua...) 
(continuação...)

Tabela 10: Dados Coletados dos Animais no Período de 31/08/93 a 31/08/94.

\begin{tabular}{|c|c|c|c|c|c|c|c|c|c|c|}
\hline Data & Estações & Atendim. & Idade & Sexo & Diárias & óbito & $\begin{array}{l}\text { Temper. } \\
\text { Máxima }\end{array}$ & $\begin{array}{l}\text { Temper. } \\
\text { Minima }\end{array}$ & $\begin{array}{l}\text { Anplitude } \\
\text { Temper. }\end{array}$ & Umidade \\
\hline $16 / 11 / 93$ & 0 & 4 & 3 & 1 & 5 & 0 & 31,8 & 15,9 & 15,9 & 55,1 \\
\hline $16 / 11 / 93$ & 0 & 4 & 3 & 0 & 2 & 1 & 31,8 & 15,9 & 15,9 & 55,1 \\
\hline $16 / 11 / 93$ & 0 & 1 & 72 & 1 & 0 & 1 & 31,8 & 15,9 & 15,9 & 55,1 \\
\hline $17 / 11 / 93$ & 0 & 1 & 6 & 0 & 0 & 0 & 31,6 & 17,3 & 14,3 & 54,9 \\
\hline $17 / 11 / 93$ & 0 & 5 & 2 & 1 & 4 & 0 & 31,6 & 17,3 & 14,3 & 54,9 \\
\hline $17 / 11 / 93$ & 0 & 4 & 2 & 1 & 4 & 1 & 31,6 & 17,3 & 14,3 & 54,9 \\
\hline $18 / 11 / 93$ & 0 & 1 & 2,3 & 1 & 0 & 0 & 31,6 & 17,4 & 14,2 & 58,3 \\
\hline $18 / 11 / 93$ & 0 & 2 & 7 & 1 & 4 & 0 & 31,6 & 17,4 & 14,2 & 58,3 \\
\hline $18 / 11 / 93$ & 0 & 1 & 3 & 1 & 0 & 0 & 31,6 & 17,4 & 14,2 & 58,3 \\
\hline $18 / 11 / 93$ & 0 & 1 & 5 & 1 & 4 & 1 & 31,6 & 17,4 & 14,2 & 58,3 \\
\hline $19 / 11 / 93$ & 0 & 1 & 1,5 & 0 & 5 & 0 & 33,1 & 20,6 & 12,5 & 57,0 \\
\hline $19 / 11 / 93$ & 0 & 1 & 7 & 0 & 2 & 0 & 33,1 & 20,6 & 12,5 & 57,0 \\
\hline $19 / 11 / 93$ & 0 & 1 & 3 & 0 & 4 & 1 & 33,1 & 20,6 & 12,5 & 57,0 \\
\hline $20 / 11 / 93$ & 0 & 1 & 6 & 0 & 0 & 0 & 34,5 & 19,5 & 15,0 & 38,3 \\
\hline $20 / 11 / 93$ & 0 & 1 & 8 & 1 & 1 & 1 & 34,5 & 19,5 & 15,0 & 38,3 \\
\hline $20 / 11 / 93$ & 0 & 1 & 7 & 1 & 2 & 1 & 34,5 & 19,5 & 15,0 & 38,3 \\
\hline $21 / 11 / 93$ & 0 & 3 & 7 & 1 & 3 & 0 & 34,9 & 19,4 & 15,5 & 39,6 \\
\hline $21 / 11 / 93$ & 0 & 1 & 5 & 1 & 1 & 1 & 34,9 & 19,4 & 15,5 & 39,6 \\
\hline $22 / 11 / 93$ & 0 & 1 & 3 & 0 & 0 & 0 & 35,4 & 18,7 & 16,7 & 39,7 \\
\hline $22 / 11 / 93$ & 0 & 1 & 4 & 0 & 3 & 0 & 35,4 & 18,7 & 16,7 & 39,7 \\
\hline $23 / 11 / 93$ & 0 & 1 & 2 & 0 & 3 & 0 & 35,8 & 20,0 & 15,8 & 37,7 \\
\hline $23 / 11 / 93$ & 0 & 1 & 7 & 1 & 4 & $\mathbf{0}$ & 35,8 & 20,0 & 15,8 & 37,7 \\
\hline $23 / 11 / 93$ & 0 & 1 & 6 & 0 & 2 & 1 & 35,8 & 20,0 & 15,8 & 37,7 \\
\hline $24 / 11 / 93$ & 0 & 1 & 4 & 0 & 0 & 0 & 28,8 & 21,9 & 6,9 & 84,2 \\
\hline $26 / 11 / 93$ & 0 & 1 & 5 & 0 & 3 & $\mathbf{0}$ & 30,9 & 17,7 & 13,2 & 48,7 \\
\hline $27 / 11 / 93$ & 0 & 1 & 3 & 0 & 4 & 0 & 31,8 & 15,9 & 15,9 & 47,3 \\
\hline $27 / 11 / 93$ & 0 & 1 & 36 & 0 & 0 & 0 & 31,8 & 15,9 & 15,9 & 47,3 \\
\hline $28 / 11 / 93$ & 0 & 1 & 3 & 0 & 0 & 0 & 32,4 & 17,7 & 14,7 & 42,9 \\
\hline $29 / 11 / 93$ & 0 & 1 & 5 & 0 & 0 & o & 33,4 & 17,0 & 16,4 & 53,2 \\
\hline $29 / 11 / 93$ & 0 & 1 & 1,7 & 0 & 0 & 0 & 33,4 & 17,0 & 16,4 & 53,2 \\
\hline $29 / 11 / 93$ & 0 & 1 & 4 & 1 & 4 & 0 & 33,4 & 17,0 & 16,4 & 53,2 \\
\hline $29 / 11 / 93$ & 0 & 1 & 3 & 1 & 0 & 0 & 33,4 & 17,0 & 16,4 & 53,2 \\
\hline $29 / 11 / 93$ & 0 & 1 & 8 & 1 & 0 & 0 & 33,4 & 17,0 & 16,4 & 53,2 \\
\hline $29 / 11 / 93$ & 0 & 1 & 8 & 1 & 2 & 1 & 33,4 & 17,0 & 16,4 & 53,2 \\
\hline $30 / 11 / 93$ & 0 & 1 & 60 & 0 & 0 & 0 & 34,4 & 21,0 & 13,4 & 68,6 \\
\hline $30 / 11 / 93$ & 0 & 1 & 6 & 1 & 6 & $\mathbf{0}$ & 34,4 & 21,0 & 13,4 & 68,6 \\
\hline $30 / 11 / 93$ & 0 & 1 & 9 & 1 & 7 & 0 & 34,4 & 21,0 & 13,4 & 68,6 \\
\hline $01 / 12 / 93$ & 0 & 1 & 4 & 0 & 0 & 0 & 30,3 & 19,2 & 11,1 & 70,8 \\
\hline
\end{tabular}


(continuação...)

Tabela 10: Dados Coletados dos Animais no Período de 31/08/93 a 31/08/94.

\begin{tabular}{|c|c|c|c|c|c|c|c|c|c|c|}
\hline Data & Estações & Atendia. & Idade & Sexo & Diárias & óbito & $\begin{array}{l}\text { Temper. } \\
\text { Máxina }\end{array}$ & $\begin{array}{l}\text { Temper. } \\
\text { Mínima }\end{array}$ & $\begin{array}{l}\text { Araplitude } \\
\text { Temper. }\end{array}$ & Umidade \\
\hline $01 / 12 / 93$ & 0 & 1 & 4,2 & 1 & 0 & 0 & 30,3 & 19,2 & 11,1 & 70,8 \\
\hline $01 / 12 / 93$ & 0 & 1 & 6 & 1 & 1 & 1 & 30,3 & 19,2 & 11,1 & 70,8 \\
\hline $02 / 12 / 93$ & 0 & 1 & 5 & 0 & 0 & 0 & 28,5 & 20,3 & 8,2 & 88,6 \\
\hline $02 / 12 / 93$ & 0 & 1 & 2 & 0 & 3 & 0 & 28,5 & 20,3 & 8,2 & 88,6 \\
\hline $03 / 12 / 93$ & 0 & 2 & 2,4 & 1 & 3 & 0 & 26,6 & 20,4 & 6,2 & 80,2 \\
\hline $03 / 12 / 93$ & 0 & 1 & 7 & 1 & 3 & 1 & 26,6 & 20,4 & 6,2 & 80,2 \\
\hline $04 / 12 / 93$ & 0 & 3 & 3 & 0 & 3 & 0 & 28,6 & 18,3 & 10,3 & 63,2 \\
\hline $04 / 12 / 93$ & 0 & 2 & 3 & 0 & 3 & 0 & 28,6 & 18,3 & 10,3 & 63,2 \\
\hline $04 / 12 / 93$ & 0 & 2 & 24 & 1 & 0 & 0 & 28,6 & 18,3 & 10,3 & 63,2 \\
\hline $06 / 12 / 93$ & 0 & 1 & 5 & 1 & 0 & 0 & 32,6 & 15,3 & 17,3 & 41,7 \\
\hline $06 / 12 / 93$ & 0 & 1 & 3 & 0 & 1 & 1 & 32,6 & 15,3 & 17,3 & 41,7 \\
\hline $08 / 12 / 93$ & 0 & 1 & 6 & 1 & 0 & 0 & 29,1 & 19,2 & 9,9 & 73,7 \\
\hline $08 / 12 / 93$ & 0 & 1 & 5 & 1 & 0 & 0 & 29,1 & 19,2 & 9,9 & 73,7 \\
\hline $09 / 12 / 93$ & 0 & 1 & 48 & 0 & 0 & 0 & 34,0 & 18,6 & 15,4 & 64,9 \\
\hline $09 / 12 / 93$ & 0 & 1 & 24 & 1 & 0 & 0 & 34,0 & 18,6 & 15,4 & 64,9 \\
\hline $09 / 12 / 93$ & 0 & 1 & 7 & 1 & 0 & 0 & 34,0 & 18,6 & 15,4 & 64,9 \\
\hline $10 / 12 / 93$ & 0 & 2 & 2 & 1 & 4 & 0 & 34,6 & 21,8 & 12,8 & 63,5 \\
\hline $10 / 12 / 93$ & 0 & 1 & 4 & 1 & 3 & 1 & 34,6 & 21,8 & 12,8 & 63,5 \\
\hline $11 / 12 / 93$ & 0 & 1 & 4,5 & 1 & 4 & 1 & 35,2 & 21,0 & 14,2 & 56,0 \\
\hline $13 / 12 / 93$ & 0 & 2 & 4 & 0 & 3 & 1 & 31,9 & 18,9 & 13,0 & 71,2 \\
\hline $14 / 12 / 93$ & 0 & 1 & 2 & 0 & 0 & 0 & 32,9 & 20,5 & 12,4 & 64,1 \\
\hline $16 / 12 / 93$ & 0 & 1 & 1 & 0 & 0 & 0 & 32,2 & 19,8 & 12,4 & 80,5 \\
\hline $16 / 12 / 93$ & 0 & 1 & 3 & 1 & 0 & 0 & 32,2 & 19,8 & 12,4 & 80,5 \\
\hline $17 / 12 / 93$ & 0 & 1 & 5 & 0 & 0 & 0 & 28,2 & 19,3 & 8,9 & 87,6 \\
\hline $17 / 12 / 93$ & 0 & 3 & 1,5 & 1 & 4 & 0 & 28,2 & 19,3 & 8,9 & 87,6 \\
\hline $18 / 12 / 93$ & 0 & 1 & 8 & 0 & 5 & 0 & 26,1 & 20,5 & 5,6 & 91,2 \\
\hline $19 / 12 / 93$ & 0 & 5 & 10 & 0 & 0 & 1 & 28,6 & 18,9 & 9,7 & 67,9 \\
\hline $20 / 12 / 93$ & 0 & 1 & 4 & 1 & 1 & 1 & 30,4 & 19,1 & 11,3 & 73,8 \\
\hline $21 / 12 / 93$ & 1 & 3 & 4 & 1 & 4 & 1 & 29,2 & 20,9 & 8,3 & 92,4 \\
\hline $21 / 12 / 93$ & 1 & 1 & 4 & 1 & 1 & 1 & 29,2 & 20,9 & 8,3 & 92,4 \\
\hline $22 / 12 / 93$ & 1 & 1 & 5 & 0 & 1 & 1 & 28,0 & 21,1 & 6,9 & 91,3 \\
\hline $23 / 12 / 93$ & 1 & 1 & 48 & 0 & 0 & 0 & 27,4 & 21,1 & 6,3 & 93,6 \\
\hline $26 / 12 / 93$ & 1 & 1 & 2 & 1 & 2 & 0 & 29,9 & 18,0 & 11,9 & 72,4 \\
\hline $27 / 12 / 93$ & 1 & 1 & 14 & 0 & 0 & 0 & 30,4 & 22,0 & 8,4 & 68,0 \\
\hline $28 / 12 / 93$ & 1 & 1 & 12 & 0 & 0 & 0 & 28,4 & 19,1 & 9,3 & 61,9 \\
\hline $28 / 12 / 93$ & 1 & 1 & 1 & 1 & 1 & 1 & 28,4 & 19,1 & 9,3 & 61,9 \\
\hline $29 / 12 / 93$ & 1 & 2 & 4 & 1 & 2 & 1 & 29,3 & 15,6 & 13,7 & 55,2 \\
\hline $30 / 12 / 93$ & 1 & 2 & 5 & 1 & 6 & 0 & 29,4 & 17,0 & 12,4 & 55,4 \\
\hline $02 / 01 / 94$ & 1 & 1 & 1,5 & 0 & 0 & 0 & 28,0 & 19,0 & 9,0 & 87,3 \\
\hline
\end{tabular}


(continuação...)

Tabela 10: Dados Coletados dos Animais no Período de 31/08/93 a 31/08/94.

\begin{tabular}{|c|c|c|c|c|c|c|c|c|c|c|}
\hline Data & Estações & Atenditi. & Idade & Sexo & Diárias & obito & $\begin{array}{l}\text { Temper. } \\
\text { Máxima }\end{array}$ & $\begin{array}{l}\text { Temper. } \\
\text { Mínima }\end{array}$ & $\begin{array}{l}\text { Amplitude } \\
\text { Temper. }\end{array}$ & Umidade \\
\hline $02 / 01 / 94$ & 1 & 1 & 4 & 1 & 0 & 0 & 28,0 & 19,0 & 9,0 & 87,3 \\
\hline $02 / 01 / 94$ & 1 & 1 & 36 & 1 & 0 & 1 & 28,0 & 19,0 & 9,0 & 87,3 \\
\hline $02 / 01 / 94$ & 1 & 3 & 2 & 1 & 2 & 1 & 28,0 & 19,0 & 9,0 & 87,3 \\
\hline $03 / 01 / 94$ & 1 & 1 & 7 & 0 & 1 & 0 & 29,0 & 19,4 & 9,6 & 79,1 \\
\hline $03 / 01 / 94$ & 1 & 1 & 16 & 1 & 0 & 0 & 29,0 & 19,4 & 9,6 & 79,1 \\
\hline $03 / 01 / 94$ & 1 & 1 & 3 & 0 & 0 & 1 & 29,0 & 19,4 & 9,6 & 79,1 \\
\hline $04 / 01 / 94$ & 1 & 1 & 4 & 1 & 0 & 0 & 24,6 & 19,2 & 5,4 & 93,0 \\
\hline $04 / 01 / 94$ & 1 & 1 & 2 & 0 & 1 & 1 & 24,6 & 19,2 & 5,4 & 93,0 \\
\hline $04 / 01 / 94$ & 1 & 1 & 4 & 1 & 0 & 1 & 24,6 & 19,2 & 5,4 & 93,0 \\
\hline $05 / 01 / 94$ & 1 & 2 & 2 & 0 & 2 & 0 & 28,0 & 18,7 & 9,3 & 78,5 \\
\hline $05 / 01 / 94$ & 1 & 1 & 18 & 1 & 0 & 0 & 28,0 & 18,7 & 9,3 & 78,5 \\
\hline $06 / 01 / 94$ & 1 & 1 & 6 & 0 & 0 & 0 & 29,5 & 17,7 & 11,8 & 68,9 \\
\hline $06 / 01 / 94$ & 1 & 1 & 6 & 1 & 0 & 0 & 29,5 & 17,7 & 11,8 & 68,9 \\
\hline $06 / 01 / 94$ & 1 & 1 & 2 & 1 & 6 & 0 & 29,5 & 17,7 & 11,8 & 68,9 \\
\hline $06 / 01 / 94$ & 1 & 1 & 2 & 1 & 2 & 1 & 29,5 & 17,7 & 11,8 & 68,9 \\
\hline $08 / 01 / 94$ & 1 & 1 & 3 & 1 & 0 & 0 & 31,4 & 17,0 & 14,4 & 58,9 \\
\hline $08 / 01 / 94$ & 1 & 1 & 4 & 1 & 1 & 1 & 31,4 & 17,0 & 14,4 & 58,9 \\
\hline $09 / 01 / 94$ & 1 & 1 & 4 & 0 & 0 & 0 & 32,8 & 18,6 & 14,2 & 73,0 \\
\hline $10 / 01 / 94$ & 1 & 1 & 6 & 1 & 0 & 0 & 25,2 & 19,5 & 5,7 & 83,0 \\
\hline $10 / 01 / 94$ & 1 & 1 & 3 & 1 & 0 & 0 & 25,2 & 19,5 & 5,7 & 83,0 \\
\hline $10 / 01 / 94$ & 1 & 2 & 3 & 0 & 1 & 1 & 25,2 & 19,5 & 5,7 & 83,0 \\
\hline $12 / 01 / 94$ & 1 & 1 & 2 & 0 & 2 & 0 & 29,2 & 17,5 & 11,7 & 57,3 \\
\hline $13 / 01 / 94$ & 1 & 1 & 6 & 0 & 0 & 0 & 29,4 & 15,6 & 13,8 & 62,9 \\
\hline $13 / 01 / 94$ & 1 & 1 & 1,5 & 0 & 0 & 0 & 29,4 & 15,6 & 13,8 & 62,9 \\
\hline $13 / 01 / 94$ & 1 & 1 & 2 & 1 & 0 & 0 & 29,4 & 15,6 & 13,8 & 62,9 \\
\hline $13 / 01 / 94$ & 1 & 1 & 3 & 0 & 2 & 1 & 29,4 & 15,6 & 13,8 & 62,9 \\
\hline $13 / 01 / 94$ & 1 & 1 & 4 & 0 & 2 & 1 & 29,4 & 15,6 & 13,8 & 62,9 \\
\hline $14 / 01 / 94$ & 1 & 1 & 4 & 0 & 4 & 0 & 29,0 & 19,4 & 9,6 & 81,6 \\
\hline $15 / 01 / 94$ & 1 & 1 & 3 & 1 & 0 & 0 & 22,8 & 20,8 & 2,0 & 92,7 \\
\hline $15 / 01 / 94$ & 1 & 1 & 3 & 1 & 0 & 0 & 22,8 & 20,8 & 2,0 & 92,7 \\
\hline $16 / 01 / 94$ & 1 & 1 & 5 & 1 & 3 & 0 & 26,0 & 20,2 & 5,8 & 84,4 \\
\hline $17 / 01 / 94$ & 1 & 1 & 2 & 0 & 3 & 0 & 27,2 & 14,7 & 12,5 & 58,6 \\
\hline $17 / 01 / 94$ & 1 & 1 & 4 & 1 & 2 & 0 & 27,2 & 14,7 & 12,5 & 58,6 \\
\hline $18 / 01 / 94$ & 1 & 2 & 2 & 0 & 0 & 0 & 28,6 & 14,7 & 13,9 & 53,1 \\
\hline $18 / 01 / 94$ & 1 & 1 & 6 & 1 & 1 & 1 & 28,6 . & 14,7 & 13,9 & 53,1 \\
\hline $19 / 01 / 94$ & 1 & 1 & 48 & 1 & 0 & 0 & 30,2 & 16,8 & 13,4 & 56,1 \\
\hline $19 / 01 / 94$ & 1 & 1 & 3 & 1 & 0 & 0 & 30,2 & 16,8 & 13,4 & 56,1 \\
\hline $19 / 01 / 94$ & 1 & 1 & 2 & 1 & 1 & 1 & $30 ; 2$ & 16,8 & 13,4 & 56,1 \\
\hline
\end{tabular}


(continuação...)

Tabela 10: Dados Coletados dos Animais no Período de 31/08/93 a 31/08/94.

\begin{tabular}{|c|c|c|c|c|c|c|c|c|c|c|}
\hline Data & Estações & Atendim. & Idade & Sexo & Diárias & óbito & $\begin{array}{l}\text { Temper. } \\
\text { Máxima }\end{array}$ & $\begin{array}{l}\text { Temper. } \\
\text { Mínima }\end{array}$ & $\begin{array}{c}\text { Amplitude } \\
\text { Terper. }\end{array}$ & Umidade \\
\hline $20 / 01 / 94$ & 1 & 1 & 5 & 0 & 5 & 0 & 30,8 & 17,2 & 13,6 & 56,3 \\
\hline $20 / 01 / 94$ & 1 & 2 & 48 & 0 & 3 & 0 & 30,8 & 17,2 & 13,6 & 56,3 \\
\hline $20 / 01 / 94$ & 1 & 2 & 3 & 1 & 0 & 0 & 30,8 & 17,2 & 13,6 & 56,3 \\
\hline $21 / 01 / 94$ & 1 & 1 & 8 & 0 & 0 & 0 & 29,3 & 19,0 & 10,3 & 68,7 \\
\hline $21 / 0 t / 94$ & 1 & 1 & 9 & 1 & 2 & 0 & 29,3 & 19,0 & 10,3 & 68,7 \\
\hline $21 / 01 / 94$ & 1 & 1 & 37 & 1 & 1 & 1 & 29,3 & 19,0 & 10,3 & 68,7 \\
\hline $22 / 01 / 94$ & 1 & 1 & 7 & 0 & 0 & 0 & 28,6 & 16,2 & 12,4 & 72,6 \\
\hline $22 / 01 / 94$ & 1 & 5 & 4 & 0 & 0 & 0 & 28,6 & 16,2 & 12,4 & 72,6 \\
\hline $22 / 01 / 94$ & 1 & 4 & 2 & 1 & 0 & 0 & 28,6 & 16,2 & 12,4 & 72,6 \\
\hline $22 / 01 / 94$ & 1 & 3 & 3 & 1 & 4 & 0 & 28,6 & 16,2 & 12,4 & 72,6 \\
\hline $31 / 01 / 94$ & 1 & 2 & 2 & 0 & 1 & 1 & 30,1 & 22,0 & 8,1 & 80,8 \\
\hline $03 / 02 / 94$ & 1 & 1 & 6 & 0 & 0 & 0 & 25,6 & 20,8 & 4,8 & 97,9 \\
\hline $04 / 02 / 94$ & 1 & 1 & 3 & 1 & 2 & $\mathbf{0}$ & 25,5 & 19,2 & 6,3 & 95,2 \\
\hline $04 / 02 / 94$ & 1 & 1 & 3 & 1 & 2 & $\mathbf{0}$ & 25,5 & 19,2 & 6,3 & 95,2 \\
\hline $05 / 02 / 94$ & 1 & 1 & 4 & 1 & 0 & $\mathbf{0}$ & 28,2 & 20,1 & 8,1 & 88,0 \\
\hline $06 / 02 / 94$ & 1 & 1 & 2 & 1 & 0 & 0 & 31,0 & 19,1 & 11,9 & 81,0 \\
\hline $06 / 02 / 94$ & 1 & 1 & 4 & 0 & 2 & 1 & 31,0 & 19,1 & 11,9 & 81,0 \\
\hline $07 / 02 / 94$ & 1 & 2 & 2 & 0 & 0 & 0 & 27,3 & 19,9 & 7,4 & 80,8 \\
\hline $07 / 02 / 94$ & 1 & 2 & 2 & 0 & 5 & 0 & 27,3 & 19,9 & 7,4 & 80,8 \\
\hline $07 / 02 / 94$ & 1 & 1 & 3 & 1 & 1 & 0 & 27,3 & 19,9 & 7,4 & 80,8 \\
\hline $07 / 02 / 94$ & 1 & 1 & 19 & 1 & 1 & $\mathbf{0}$ & 27,3 & 19,9 & 7,4 & 80,8 \\
\hline $07 / 02 / 94$ & 1 & 1 & 4 & 1 & 0 & 0 & 27,3 & 19,9 & 7,4 & 80,8 \\
\hline $07 / 02 / 94$ & 1 & 1 & 4 & 1 & 0 & 1 & 27,3 & 19,9 & 7,4 & 80,8 \\
\hline $08 / 02 / 94$ & 1 & 1 & 4 & 0 & 2 & 0 & 32,2 & 20,6 & 11,6 & 65,7 \\
\hline $08 / 02 / 94$ & 1 & 3 & 15 & 0 & 3 & 0 & 32,2 & 20,6 & 11,6 & 65,7 \\
\hline $08 / 02 / 94$ & 1 & 1 & 5 & 0 & 3 & 0 & 32,2 & 20,6 & 11,6 & 65,7 \\
\hline $08 / 02 / 94$ & 1 & 1 & 3 & 1 & 0 & 0 & 32,2 & 20,6 & 11,6 & 65,7 \\
\hline $08 / 02 / 94$ & 1 & 1 & 4 & 1 & 1 & 1 & 32,2 & 20,6 & 11,6 & 65,7 \\
\hline $09 / 02 / 94$ & 1 & 1 & 5 & 1 & 0 & 0 & 32,5 & 20,7 & 11,8 & 81,8 \\
\hline $10 / 02 / 94$ & 1 & 2 & 4 & 1 & 1 & 1 & 32,4 & 19,3 & 13,1 & 63,8 \\
\hline $10 / 02 / 94$ & 1 & 1 & 2 & 1 & 3 & 1 & 32,4 & 19,3 & 13,1 & 63,8 \\
\hline $12 / 02 / 94$ & 1 & 1 & 4 & 1 & 0 & $\mathbf{0}$ & 32,1 & 20,3 & 11,8 & 85,3 \\
\hline $12 / 02 / 94$ & 1 & 1 & 4 & 1 & 0 & 0 & 32,1 & 20,3 & 11,8 & 85,3 \\
\hline $13 / 02 / 94$ & 1 & 1 & 3 & 0 & 8 & $\mathbf{0}$ & 28,8 & 21,0 & 7,8 & 86,6 \\
\hline $13 / 02 / 94$ & 1 & 1 & 3 & 0 & 0 & o & 28,8 & 21,0 & 7,8 & 86,6 \\
\hline $13 / 02 / 94$ & 1 & 1 & 4 & 0 & 1 & 1 & 28,8 & 21,0 & 7,8 & 86,6 \\
\hline $14 / 02 / 94$ & 1 & 1 & 2 & 0 & 0 & 0 & 27,2 & 19,2 & 8,0 & 82,1 \\
\hline $14 / 02 / 94$ & 1 & 4 & 2 & 1 & 5 & $\mathbf{0}$ & 27,2 & 19,2 & 8,0 & 82,1 \\
\hline $14 / 02 / 94$ & 1 & 1 & 3 & 1 & 0 & 0 & 27,2 & 19,2 & 8,0 & 82,1 \\
\hline
\end{tabular}

(continua...) 
(continuação...)

Tabela 10: Dados Coletados dos Animais no Período de 31/08/93 a 31/08/94.

\begin{tabular}{|c|c|c|c|c|c|c|c|c|c|c|}
\hline Data & Estações & Atendir. & Idade & Sexo & Diárias & óbito & $\begin{array}{l}\text { Temper. } \\
\text { Máxima }\end{array}$ & $\begin{array}{l}\text { Teaper. } \\
\text { Mínima }\end{array}$ & $\begin{array}{l}\text { Amplitude } \\
\text { Temper. }\end{array}$ & Umidade \\
\hline $14 / 02 / 94$ & 1 & 1 & 4 & 1 & 2 & 0 & 27,2 & 19,2 & 8,0 & 82,1 \\
\hline $14 / 02 / 94$ & 1 & 1 & 3 & 1 & 1 & 1 & 27,2 & 19,2 & 8,0 & 82,1 \\
\hline $14 / 02 / 94$ & 1 & 2 & 3 & 1 & 0 & 1 & 27,2 & 19,2 & 8,0 & 82,1 \\
\hline $15 / 02 / 94$ & 1 & 1 & 4 & 0 & 0 & 0 & 30,2 & 19,2 & 11,0 & 72,4 \\
\hline $15 / 02 / 94$ & 1 & 1 & 3 & 0 & $\mathbf{0}$ & 0 & 30,2 & 19,2 & 11,0 & 72,4 \\
\hline $15 / 02 / 94$ & 1 & 1 & 2 & 0 & 2 & 0 & 30,2 & 19,2 & 11,0 & 72,4 \\
\hline $15 / 02 / 94$ & 1 & 5 & 7 & 0 & 3 & 0 & 30,2 & $\uparrow 9,2$ & 11,0 & 72,4 \\
\hline $15 / 02 / 94$ & 1 & 2 & 3 & 1 & 1 & 0 & 30,2 & 19,2 & 11,0 & 72,4 \\
\hline $16 / 02 / 94$ & 1 & 1 & 8 & 0 & 7 & 0 & 32,1 & 20,4 & 11,7 & 74,5 \\
\hline $16 / 02 / 94$ & 1 & 1 & 3 & 0 & 4 & 0 & 32,1 & 20,4 & 11,7 & 74,5 \\
\hline $16 / 02 / 94$ & 1 & 1 & 3 & 1 & 1 & 1 & 32,1 & 20,4 & 11,7 & 74,5 \\
\hline $17 / 02 / 94$ & 1 & 1 & 3 & 0 & 5 & 0 & 32,1 & 21,1 & 11,0 & 75,2 \\
\hline $17 / 02 / 94$ & 1 & 1 & 3 & 1 & 5 & 0 & 32,1 & 21,1 & 11,0 & 75,2 \\
\hline $17 / 02 / 94$ & 1 & 1 & 5 & 1 & 2 & 0 & 32,1 & 21,1 & 11,0 & 75,2 \\
\hline $17 / 02 / 94$ & 1 & 5 & 2 & 1 & 4 & o & 32,1 & 21,1 & 11,0 & 75,2 \\
\hline $18 / 02 / 94$ & 1 & 1 & 4 & 0 & 0 & 0 & 32,0 & 21,1 & 10,9 & 74,0 \\
\hline $19 / 02 / 94$ & 1 & 1 & 2 & 1 & 7 & 0 & 30,1 & 21,1 & 8,9 & 84,4 \\
\hline $19 / 02 / 94$ & 1 & 1 & 3 & 0 & 1 & 1 & 30,1 & 21,1 & 8,9 & 84,4 \\
\hline $19 / 02 / 94$ & 1 & 6 & 4 & 1 & 6 & 1 & 30,1 & 21,1 & 8,9 & 84,4 \\
\hline $20 / 02 / 94$ & 1 & 1 & 2 & 0 & 1 & 0 & 31,2 & 20,0 & 11,2 & 81,0 \\
\hline $20 / 02 / 94$ & 1 & 1 & 7 & 0 & 11 & 0 & 31,2 & 20,0 & 11,2 & 81,0 \\
\hline $20 / 02 / 94$ & 1 & 2 & 4 & 1 & 1 & 0 & 31,2 & 20,0 & 11,2 & 81,0 \\
\hline $20 / 02 / 94$ & 1 & 1 & 4 & 0 & 1 & 1 & 31,2 & 20,0 & 11,2 & 81,0 \\
\hline $20 / 02 / 94$ & 1 & 1 & 4 & 0 & 1 & 1 & 31,2 & 20,0 & 11,2 & 81,0 \\
\hline $20 / 02 / 94$ & 1 & 2 & 4 & 1 & 1 & 1 & 31,2 & 20,0 & 11,2 & 81,0 \\
\hline $21 / 02 / 94$ & 1 & 2 & 5 & 1 & 3 & 0 & 31,0 & 20,8 & 10,2 & 82,3 \\
\hline $21 / 02 / 94$ & 1 & 1 & 3 & 1 & 1 & 0 & 31,0 & 20,8 & 10,2 & 82,3 \\
\hline $21 / 02 / 94$ & 1 & 1 & 4 & 0 & 2 & 1 & 31,0 & 20,8 & 10,2 & 82,3 \\
\hline $21 / 02 / 94$ & 1 & 1 & 96 & 1 & 1 & 1 & 31,0 & 20,8 & 10,2 & 82,3 \\
\hline $21 / 02 / 94$ & 1 & 1 & 3 & 1 & 4 & 1 & 31,0 & 20,8 & 10,2 & 82,3 \\
\hline $22 / 02 / 94$ & 1 & 3 & 2 & 1 & 3 & 0 & 30,8 & 20,6 & 10,2 & 83,0 \\
\hline $22 / 02 / 94$ & 1 & 1 & 2 & 1 & 3 & 0 & 30,8 & 20,6 & 10,2 & 83,0 \\
\hline $22 / 02 / 94$ & 1 & 1 & 3 & 1 & 0 & 0 & 30,8 & 20,6 & 10,2 & 83,0 \\
\hline $22 / 02 / 94$ & 1 & 1 & 3 & 0 & 3 & 1 & 30,8 & 20,6 & 10,2 & 83,0 \\
\hline $23 / 02 / 94$ & 1 & 1 & 3 & 1 & 3 & 0 & 31,6 & 20,6 & 11,0 & 73,6 \\
\hline $23 / 02 / 94$ & 1 & 1 & 8 & 1 & 3 & 0 & 31,6 & 20,6 & 11,0 & 73,6 \\
\hline $23 / 02 / 94$ & 1 & 1 & 3 & 0 & 1 & 1 & 31,6 & 20,6 & 11,0 & 73,6 \\
\hline $23 / 02 / 94$ & 1 & 1 & 2 & 0 & 3 & 1 & 31,6 & 20,6 & 11,0 & 73,6 \\
\hline $23 / 02 / 94$ & 1 & 1 & 5 & 0 & 2 & 1 & 31,6 & 20,6 & 11,0 & 73,6 \\
\hline
\end{tabular}


(continuação...)

Tabela 10: Dados Coletados dos Animais no Período de 31/08/93 a 31/08/94.

\begin{tabular}{|c|c|c|c|c|c|c|c|c|c|c|}
\hline Data & Estações & Atendim. & Idade & Sexo & Diárias & óbito & $\begin{array}{l}\text { Teaper. } \\
\text { Máxima }\end{array}$ & $\begin{array}{l}\text { Temper. } \\
\text { Mínima }\end{array}$ & $\begin{array}{l}\text { Amplitude } \\
\text { Tenper. }\end{array}$ & Umidade \\
\hline $23 / 02 / 94$ & 1 & 1 & 2 & 1 & 1 & 1 & 31,6 & 20,6 & 11,0 & 73,6 \\
\hline $23 / 02 / 94$ & 1 & 1 & 1 & 1 & 3 & 1 & 31,6 & 20,6 & 11,0 & 73,6 \\
\hline $25 / 02 / 94$ & 1 & 1 & 5 & 1 & 7 & 0 & 32,4 & 20,6 & 11,8 & 79,2 \\
\hline $25 / 02 / 94$ & 1 & 1 & 5 & 0 & 1 & 1 & 32,4 & 20,6 & 11,8 & 79,2 \\
\hline $26 / 02 / 94$ & 1 & 1 & 2 & 0 & 0 & 0 & 30,4 & 20,3 & 10,1 & 82,4 \\
\hline $26 / 02 / 94$ & 1 & 3 & 7 & 1 & 0 & 0 & 30,4 & 20,3 & 10,1 & 82,4 \\
\hline $27 / 02 / 94$ & 1 & 1 & 4 & 0 & 0 & 0 & 31,2 & 20,1 & 11,1 & 78,0 \\
\hline $01 / 03 / 94$ & 1 & 1 & 5 & 0 & 0 & 0 & 30,5 & 19,8 & 10,7 & 75,4 \\
\hline $01 / 03 / 94$ & 1 & 1 & 3 & 0 & 2 & 0 & 30,5 & 19,8 & 10,7 & 75,4 \\
\hline $02 / 03 / 94$ & 1 & 2 & 2 & 0 & 3 & 0 & 30,1 & 20,7 & 9,4 & 80,2 \\
\hline $02 / 03 / 94$ & 1 & 1 & 6 & 0 & 0 & 0 & 30,1 & 20,7 & 9,4 & 80,2 \\
\hline $02 / 03 / 94$ & 1 & 2 & 4 & 1 & 1 & 1 & 30,1 & 20,7 & 9,4 & 80,2 \\
\hline $03 / 03 / 94$ & 1 & 1 & 36 & 1 & 3 & 0 & 27,4 & 21,2 & 6,2 & 92,9 \\
\hline $04 / 03 / 94$ & 1 & 2 & 2 & 1 & 5 & 0 & 29,4 & 20,0 & 9,4 & 93,6 \\
\hline $05 / 03 / 94$ & 1 & 1 & 48 & 0 & 2 & 0 & 29,9 & 20,4 & 9,5 & 80,3 \\
\hline $05 / 03 / 94$ & 1 & 4 & 4 & 0 & 2 & $\mathbf{0}$ & 29,9 & 20,4 & 9,5 & 80,3 \\
\hline $05 / 03 / 94$ & 1 & 1 & 11 & 1 & 2 & 0 & 29,9 & 20,4 & 9,5 & 80,3 \\
\hline $05 / 03 / 94$ & 1 & 1 & 5 & 1 & 5 & 0 & 29,9 & 20,4 & 9,5 & 80,3 \\
\hline $06 / 03 / 94$ & 1 & 1 & 3 & 1 & 8 & $\mathbf{0}$ & 31,2 & 21,2 & 10,0 & 74,9 \\
\hline $06 / 03 / 94$ & 1 & 2 & 6 & 1 & 2 & 0 & 31,2 & 21,2 & 10,0 & 74,9 \\
\hline $06 / 03 / 94$ & 1 & 1 & 2 & 1 & 4 & 0 & 31,2 & 21,2 & 10,0 & 74,9 \\
\hline $07 / 03 / 94$ & 1 & 1 & 18 & 0 & 1 & 0 & 29,8 & 20,9 & 8,9 & 88,6 \\
\hline $07 / 03 / 94$ & 1 & 1 & 4 & 1 & 1 & 1 & 29,8 & 20,9 & 8,9 & 88,6 \\
\hline $07 / 03 / 94$ & 1 & 1 & 3 & 1 & 1 & 1 & 29,8 & 20,9 & 8,9 & 88,6 \\
\hline $07 / 03 / 94$ & 1 & 1 & 4 & 1 & 1 & 1 & 29,8 & 20,9 & 8,9 & 88,6 \\
\hline $08 / 03 / 94$ & 1 & 1 & 4 & 0 & 4 & 0 & 25,6 & 20,6 & 5,0 & 90,2 \\
\hline $08 / 03 / 94$ & 1 & 3 & 8 & 1 & 8 & 0 & 25,6 & 20,6 & 5,0 & 90,2 \\
\hline $09 / 03 / 94$ & 1 & 1 & 4 & 0 & 2 & 0 & 25,6 & 20,6 & 5,0 & 81,2 \\
\hline $09 / 03 / 94$ & 1 & 6 & 6 & 0 & 3 & 0 & 25,6 & 20,6 & 5,0 & 81,2 \\
\hline $09 / 03 / 94$ & 1 & 2 & 15 & 0 & 2 & 1 & 25,6 & 20,6 & 5,0 & 81,2 \\
\hline $10 / 03 / 94$ & 1 & 2 & 8 & 0 & 0 & 0 & 28,2 & 18,9 & 9,3 & 67,5 \\
\hline $11 / 03 / 94$ & 1 & 1 & 8 & 0 & 1 & 1 & 28,0 & 16,2 & 11,8 & 67,7 \\
\hline $11 / 03 / 94$ & 1 & 2 & 2 & 1 & 2 & 1 & 28,0 & 16,2 & 11,8 & 67,7 \\
\hline $13 / 03 / 94$ & 1 & 1 & 7 & 0 & 0 & 0 & 27,6 & 16,0 & 11,6 & 67,9 \\
\hline $14 / 03 / 94$ & 1 & 1 & 2 & 1 & 0 & 0 & 26,3 & 15,6 & 10,7 & 80,6 \\
\hline $15 / 03 / 94$ & 1 & 1 & 96 & 1 & 1 & 0 & 25,1 & 16,3 & 8,8 & 84,6 \\
\hline $15 / 03 / 94$ & 1 & 1 & 3 & 0 & 1 & 1 & 25,1 & 16,3 & 8,8 & 84,6 \\
\hline $17 / 03 / 94$ & 1 & 1 & 3 & 0 & 5 & 0 & 30,8 & 19,4 & 11,4 & 77,7 \\
\hline $17 / 03 / 94$ & 1 & 1 & 9 & 0 & 3 & 0 & 30,8 & 19,4 & 11,4 & 77,7 \\
\hline
\end{tabular}

(continua...) 
(continuação...)

Tabela 10: Dados Coletados dos Animais no Período de 31/08/93 a 31/08/94.

\begin{tabular}{|c|c|c|c|c|c|c|c|c|c|c|}
\hline Data & Estações & Atendim. & Idade & Sexo & Diárias & óbito & $\begin{array}{l}\text { Temper. } \\
\text { Máxima }\end{array}$ & $\begin{array}{l}\text { Temper. } \\
\text { Minima }\end{array}$ & $\begin{array}{l}\text { Amplitude } \\
\text { Temper. }\end{array}$ & Umidade \\
\hline $17 / 03 / 94$ & 1 & 4 & 18 & 1 & 1 & 0 & 30,8 & 19,4 & 11,4 & 77,7 \\
\hline $18 / 03 / 94$ & 1 & 1 & 8 & 0 & 0 & 0 & 31,3 & 20,2 & 11,1 & 67,9 \\
\hline $18 / 03 / 94$ & 1 & 1 & 6 & 1 & 0 & 0 & 31,3 & 20,2 & 11,1 & 67,9 \\
\hline $18 / 03 / 94$ & 1 & 2 & 3 & 0 & 1 & 1 & 31,3 & 20,2 & 11,1 & 67,9 \\
\hline $19 / 03 / 94$ & 1 & 1 & 2 & 1 & 3 & 0 & 31,2 & 19,6 & 11,6 & 71,6 \\
\hline $20 / 03 / 94$ & 1 & 1 & 5 & 1 & 0 & 0 & 30,4 & 19,4 & 11,0 & 65,8 \\
\hline $20 / 03 / 94$ & 1 & 2 & 3 & 0 & 2 & 1 & 30,4 & 19,4 & 11,0 & 65,8 \\
\hline $21 / 03 / 94$ & 2 & 1 & 2 & 0 & 0 & 0 & 31,1 & 18,4 & 12,7 & 65,6 \\
\hline $21 / 03 / 94$ & 2 & 1 & 72 & 1 & 1 & 1 & 31,1 & 18,4 & 12,7 & 65,6 \\
\hline $22 / 03 / 94$ & 2 & 1 & 4 & 0 & 1 & 0 & 30,4 & 19,2 & 11,2 & 64,4 \\
\hline $22 / 03 / 94$ & 2 & 1 & 4 & 1 & 2 & 1 & 30,4 & 19,2 & 11,2 & 64,4 \\
\hline $23 / 03 / 94$ & 2 & 1 & 4 & 0 & 4 & 0 & 30,8 & 19,3 & 11,5 & 63,7 \\
\hline $24 / 03 / 94$ & 2 & 1 & 8 & 0 & 0 & 0 & 31,4 & 17,4 & 14,0 & 66,2 \\
\hline $24 / 03 / 94$ & 2 & 2 & 72 & 0 & 5 & 0 & 31,4 & 17,4 & 14,0 & 66,2 \\
\hline $25 / 03 / 94$ & 2 & 1 & 5 & 0 & 3 & 0 & 31,5 & 20,0 & 11,5 & 72,7 \\
\hline $25 / 03 / 94$ & 2 & 1 & 2 & 1 & 0 & 0 & 31,5 & 20,0 & 11,5 & 72,7 \\
\hline $25 / 03 / 94$ & 2 & 1 & 3 & 1 & 0 & 0 & 31,5 & 20,0 & 11,5 & 72,7 \\
\hline $26 / 03 / 94$ & 2 & 1 & 1 & 0 & 0 & 0 & 27,3 & 19,9 & 7,4 & 90,8 \\
\hline $27 / 03 / 94$ & 2 & 2 & 6 & 1 & 4 & 0 & 27,4 & 19,5 & 7,9 & 88,6 \\
\hline $28 / 03 / 94$ & 2 & 1 & 7 & 0 & 4 & 0 & 29,0 & 18,4 & 10,6 & 83,4 \\
\hline $28 / 03 / 94$ & 2 & 1 & 5 & 1 & 4 & 0 & 29,0 & 18,4 & 10,6 & 83,4 \\
\hline $28 / 03 / 94$ & 2 & 1 & 6 & 0 & 2 & 1 & 29,0 & 18,4 & 10,6 & 83,4 \\
\hline $29 / 03 / 94$ & 2 & 2 & 3 & 0 & 2 & 0 & 28,5 & 17,9 & 10,6 & 60,0 \\
\hline $29 / 03 / 94$ & 2 & 1 & 2 & 1 & 1 & 0 & 28,5 & 17,9 & 10,6 & 60,0 \\
\hline $30 / 03 / 94$ & 2 & 1 & 2 & 0 & 0 & 0 & 29,8 & 14,1 & 15,7 & 55,3 \\
\hline $31 / 03 / 94$ & 2 & 1 & 24 & 1 & 0 & 0 & 31,0 & 15,4 & 15,6 & 70,4 \\
\hline $31 / 03 / 94$ & 2 & 2 & 15 & 1 & 0 & 0 & 31,0 & 15,4 & 15,6 & 70,4 \\
\hline $01 / 04 / 94$ & 2 & 1 & 84 & 1 & 4 & 0 & 28,5 & 20,5 & 8,0 & 82,2 \\
\hline $02 / 04 / 94$ & 2 & 1 & 4 & 1 & 4 & 0 & 30,0 & 18,4 & 11,6 & 70,3 \\
\hline $02 / 04 / 94$ & 2 & 1 & 3 & 1 & 0 & 0 & 30,0 & 18,4 & 11,6 & 70,3 \\
\hline $03 / 04 / 94$ & 2 & 1 & 10 & 1 & 2 & 1 & 28,9 & 19,1 & 9,8 & 66,1 \\
\hline $04 / 04 / 94$ & 2 & 1 & 7 & 0 & 2 & 0 & 29,9 & 15,6 & 14,3 & 64,1 \\
\hline $04 / 04 / 94$ & 2 & 1 & 5 & 0 & 0 & 0 & 29,9 & 15,6 & 14,3 & 64,1 \\
\hline $04 / 04 / 94$ & 2 & 1 & 48 & 1 & 0 & 0 & 29,9 & 15,6 & 14,3 & 64,1 \\
\hline $05 / 04 / 94$ & 2 & 1 & 6 & 0 & 0 & 0 & 31,3 & 17,6 & 13,7 & 58,1 \\
\hline $05 / 04 / 94$ & 2 & 2 & 3 & 1 & 2 & 0 & 31,3 & 17,6 & 13,7 & 58,1 \\
\hline $06 / 04 / 94$ & 2 & 1 & 4 & 0 & 1 & 1 & 31,4 & 18,1 & 13,3 & 77,1 \\
\hline $07 / 04 / 94$ & 2 & 1 & 3 & 0 & 0 & 0 & 27,4 & 19,2 & 8,2 & 89,4 \\
\hline $08 / 04 / 94$ & 2 & 1 & 4 & 0 & 4 & 0 & 27,5 & 20,0 & 7,5 & 81,1 \\
\hline
\end{tabular}


(continuação...)

Tabela 10: Dados Coletados dos Animais no Período de 31/08/93 a 31/08/94.

\begin{tabular}{|c|c|c|c|c|c|c|c|c|c|c|}
\hline Data & Estações & Atendif. & Idade & Sexo & Diárias & óbito & $\begin{array}{l}\text { Temper. } \\
\text { Máxima }\end{array}$ & $\begin{array}{l}\text { Temper. } \\
\text { Mínima }\end{array}$ & $\begin{array}{l}\text { Amplitude } \\
\text { Temper. }\end{array}$ & Umidade \\
\hline $08 / 04 / 94$ & 2 & 2 & 4 & 0 & 3 & 0 & 27,5 & 20,0 & 7,5 & 81,1 \\
\hline $09 / 04 / 94$ & 2 & 1 & 2 & 1 & 5 & 1 & 28,5 & 17,4 & 11,1 & 66,2 \\
\hline $10 / 04 / 94$ & 2 & 2 & 3 & 1 & 1 & 1 & 28,3 & 14,2 & $14, t$ & 67,5 \\
\hline $11 / 04 / 94$ & 2 & 1 & 18 & 1 & 1 & 1 & 30,1 & 14,7 & 15,4 & 59,5 \\
\hline $12 / 04 / 94$ & 2 & 1 & 19 & 1 & 3 & 1 & 31,5 & 17,1 & 14,4 & 63,0 \\
\hline $13 / 04 / 94$ & 2 & 1 & 2,7 & 0 & 0 & 0 & 27,8 & 17,5 & 10,3 & 89,9 \\
\hline $14 / 04 / 94$ & 2 & 1 & 7 & 1 & 2 & 1 & 21,4 & 12,1 & 9,3 & 68,5 \\
\hline $14 / 04 / 94$ & 2 & 1 & 6 & 1 & 1 & 1 & 21,4 & 12,1 & 9,3 & 68,5 \\
\hline $15 / 04 / 94$ & 2 & 1 & 3 & 1 & 4 & 0 & 24,3 & 9,9 & 14,4 & 62,9 \\
\hline $16 / 04 / 94$ & 2 & 1 & 18 & 0 & 5 & 0 & 25,4 & 14,4 & 11,0 & 66,7 \\
\hline $18 / 04 / 94$ & 2 & 1 & 4 & 0 & 0 & 0 & 30,5 & 16,8 & 13,7 & 62,8 \\
\hline $18 / 04 / 94$ & 2 & 1 & 6 & 0 & 1 & 0 & 30,5 & 16,8 & 13,7 & 62,8 \\
\hline $18 / 04 / 94$ & 2 & 1 & 18 & 1 & 5 & 1 & 30,5 & 16,8 & 13,7 & 62,8 \\
\hline $19 / 04 / 94$ & 2 & 1 & 5 & 0 & 0 & 0 & 30,7 & 17,9 & 12,8 & 70,8 \\
\hline $19 / 04 / 94$ & 2 & 1 & 12 & 1 & 0 & $\mathbf{0}$ & 30,7 & 17,9 & 12,8 & 70,8 \\
\hline $20 / 04 / 94$ & 2 & 2 & 19 & 1 & 1 & 0 & 29,3 & 18,0 & 11,3 & 70,0 \\
\hline $21 / 04 / 94$ & 2 & 1 & 5 & 1 & 4 & 0 & 26,3 & 16,4 & 9,9 & 75,9 \\
\hline $22 / 04 / 94$ & 2 & 1 & 1,7 & 0 & 5 & 0 & 28,4 & 16,2 & 12,2 & 70,8 \\
\hline $22 / 04 / 94$ & 2 & 1 & 12 & 1 & 3 & 0 & 28,4 & 16,2 & 12,2 & 70,8 \\
\hline $23 / 04 / 94$ & 2 & 2 & 36 & 1 & 1 & 0 & 29,2 & 15,3 & 13,9 & 64,3 \\
\hline $23 / 04 / 94$ & 2 & 2 & 1,7 & 1 & 7 & 0 & 29,2 & 15,3 & 13,9 & 64,3 \\
\hline $24 / 04 / 94$ & 2 & 1 & 7 & 0 & 0 & 0 & 30,5 & 14,1 & 16,4 & 79,3 \\
\hline $24 / 04 / 94$ & 2 & 1 & 4 & 0 & 3 & 0 & 30,5 & 14,1 & 16,4 & 79,3 \\
\hline $25 / 04 / 94$ & 2 & 1 & 6 & 0 & 5 & 0 & 22,2 & 18,4 & 3,8 & 96,0 \\
\hline $27 / 04 / 94$ & 2 & 1 & 6 & 0 & 0 & 0 & 27,1 & 17,0 & 10,1 & 76,2 \\
\hline $28 / 04 / 94$ & 2 & 1 & 12 & 1 & 3 & 0 & 30,5 & 18,2 & 12,3 & 73,6 \\
\hline $30 / 04 / 94$ & 2 & 1 & 5 & 1 & 6 & 0 & 26,3 & 17,8 & 8,5 & 78,1 \\
\hline $30 / 04 / 94$ & 2 & 1 & 4 & 1 & 2 & 1 & 26,3 & 17,8 & 8,5 & 78,1 \\
\hline $01 / 05 / 94$ & 2 & 9 & 2 & 1 & 6 & 0 & 27,5 & 17,4 & 10,1 & 76,3 \\
\hline $03 / 05 / 94$ & 2 & 3 & 3 & 0 & 2 & 0 & 30,4 & 18,6 & 11,8 & 74,0 \\
\hline $03 / 05 / 94$ & 2 & 3 & 5 & 0 & 0 & 0 & 30,4 & 18,6 & 11,8 & 74,0 \\
\hline $05 / 05 / 94$ & 2 & 1 & 12 & 1 & 3 & 0 & 32,6 & 19,4 & 13,2 & 65,6 \\
\hline $05 / 05 / 94$ & 2 & 1 & 9 & 1 & 0 & 0 & 32,6 & 19,4 & 13,2 & 65,6 \\
\hline $07 / 05 / 94$ & 2 & 1 & 6 & 0 & 4 & 0 & 28,6 & 18,9 & 9,7 & 68,1 \\
\hline $07 / 05 / 94$ & 2 & 2 & 4 & 0 & 1 & 1 & 28,6 & 18,9 & 9,7 & 68,1 \\
\hline $09 / 05 / 94$ & 2 & 1 & 9 & 1 & 3 & 0 & 28,8 & 16,4 & 12,4 & 67,1 \\
\hline $10 / 05 / 94$ & 2 & 1 & 18 & 0 & 0 & 0 & 29,4 & 16,0 & 13,4 & 68,5 \\
\hline $12 / 05 / 94$ & 2 & 8 & 3 & 1 & 3 & 0 & 30,5 & 16,8 & 13,7 & 61,5 \\
\hline $12 / 05 / 94$ & 2 & 2 & 6 & 1 & 6 & 0 & 30,5 & 16,8 & 13,7 & 61,5 \\
\hline
\end{tabular}


(continuação...)

Tabela 10: Dados Coletados dos Animais no Período de 31/08/93 a 31/08/94.

\begin{tabular}{|c|c|c|c|c|c|c|c|c|c|c|}
\hline Data & Estações & Atendi明. & Idade & Sexo & Diárias & óbito & $\begin{array}{l}\text { Temper. } \\
\text { Máxima }\end{array}$ & $\begin{array}{l}\text { Temper. } \\
\text { Mínima }\end{array}$ & $\begin{array}{l}\text { Amplitude } \\
\text { Teaper. }\end{array}$ & Unidade \\
\hline $13 / 05 / 94$ & 2 & 1 & 14 & 0 & 0 & 0 & 29,1 & 16,6 & 12,5 & 66,5 \\
\hline $13 / 05 / 94$ & 2 & 1 & 5 & 0 & 2 & 1 & 29,1 & 16,6 & 12,5 & 66,5 \\
\hline $14 / 05 / 94$ & 2 & 1 & 1 & 1 & 6 & 0 & 29,8 & 16,6 & 13,2 & 66,3 \\
\hline $14 / 05 / 94$ & 2 & 1 & 6 & 1 & 1 & 1 & 29,8 & 16,6 & 13,2 & 66,3 \\
\hline $16 / 05 / 94$ & 2 & 9 & 1,3 & 0 & 7 & 1 & 30,8 & 16,8 & 14,0 & 59,0 \\
\hline $17 / 05 / 94$ & 2 & 1 & 24 & 1 & 1 & 1 & 26,5 & 18,8 & 7,7 & 77,7 \\
\hline $19 / 05 / 94$ & 2 & 6 & 4,2 & 1 & 3 & 0 & 19,8 & 17,2 & 2,6 & 92,5 \\
\hline $19 / 05 / 94$ & 2 & 2 & 5 & 1 & 2 & 1 & 19,8 & 17,2 & 2,6 & 92,5 \\
\hline $20 / 05 / 94$ & 2 & 1 & 6 & 0 & 2 & 1 & 21,8 & 13,6 & 8,2 & 84,2 \\
\hline $23 / 05 / 94$ & 2 & 1 & 6 & 0 & 0 & 0 & 22,4 & 11,0 & 11,4 & 80,2 \\
\hline $23 / 05 / 94$ & 2 & 1 & 4 & 1 & 1 & 1 & 22,4 & 11,0 & 11,4 & 80,2 \\
\hline $24 / 05 / 94$ & 2 & 1 & 4 & 0 & 3 & 0 & 24,6 & 11,2 & 13,4 & 72,3 \\
\hline $25 / 05 / 94$ & 2 & 1 & 4 & 0 & 2 & 1 & 26,3 & 11,8 & 14,5 & 65,9 \\
\hline $27 / 05 / 94$ & 2 & 2 & 5 & 0 & 5 & 0 & 24,6 & 16,4 & 8,2 & 80,5 \\
\hline $27 / 05 / 94$ & 2 & 1 & 6 & 0 & 3 & 0 & 24,6 & 16,4 & 8,2 & 80,5 \\
\hline $28 / 05 / 94$ & 2 & 1 & 7 & 1 & 4 & 0 & 27,2 & 12,8 & 14,4 & 68,2 \\
\hline $30 / 05 / 94$ & 2 & 1 & 4 & 0 & 3 & 0 & 26,1 & 16,1 & 10,0 & 77,7 \\
\hline $30 / 05 / 94$ & 2 & 1 & 30 & 0 & 0 & 0 & 26,1 & $16, t$ & 10,0 & 77,7 \\
\hline $30 / 05 / 94$ & 2 & 1 & 11 & 1 & 0 & 0 & 26,1 & 16,1 & 10,0 & 77,7 \\
\hline $30 / 05 / 94$ & 2 & 1 & 3 & 1 & 2 & 1 & 26,1 & 16,1 & 10,0 & 77,7 \\
\hline $02 / 06 / 94$ & 2 & 2 & 5 & 1 & 4 & 0 & 20,5 & 16,4 & 4,1 & 94,6 \\
\hline $02 / 06 / 94$ & 2 & 1 & 2,4 & 1 & 1 & 1 & 20,5 & 16,4 & 4,1 & 94,6 \\
\hline $03 / 06 / 94$ & 2 & 1 & 2 & 1 & 0 & 0 & 22,2 & 16,4 & 5,8 & 91,2 \\
\hline $05 / 06 / 94$ & 2 & 2 & 3 & 0 & 1 & 1 & 25,4 & 14,9 & 10,5 & 78,4 \\
\hline $06 / 06 / 94$ & 2 & 1 & 24 & 1 & 1 & 0 & 23,5 & 13,8 & 9,7 & 74,9 \\
\hline $08 / 06 / 94$ & 2 & 2 & 3 & 1 & 0 & 0 & 21,5 & 15,2 & 6,3 & 93,5 \\
\hline $08 / 06 / 94$ & 2 & 1 & 6 & 1 & 1 & 1 & 21,5 & 15,2 & 6,3 & 93,5 \\
\hline $11 / 06 / 94$ & 2 & 3 & 1,3 & 0 & 3 & 1 & 27,4 & 17,4 & 10,0 & 79,8 \\
\hline $12 / 06 / 94$ & 2 & 3 & 4 & 0 & 5 & 0 & 23,6 & 14,4 & 9,2 & 73,9 \\
\hline $14 / 06 / 94$ & 2 & 1 & 12 & 0 & 0 & 0 & 27,5 & 14,1 & 13,4 & 81,8 \\
\hline $14 / 06 / 94$ & 2 & 1 & 8 & 1 & 0 & 0 & 27,5 & 14,1 & 13,4 & 81,8 \\
\hline $16 / 06 / 94$ & 2 & 1 & 1,5 & 0 & 0 & 0 & 21,5 & 12,4 & 9,1 & 80,6 \\
\hline $19 / 06 / 94$ & 2 & 1 & 8 & 1 & 2 & 1 & 23,2 & 11,0 & 12,2 & 69,0 \\
\hline $21 / 06 / 94$ & 3 & 1 & 4 & 1 & 0 & 1 & 24,4 & 12,6 & 11,8 & 67,0 \\
\hline $24 / 06 / 94$ & 3 & 1 & 3 & 0 & 2 & 0 & 26,4 & 13,4 & 13,0 & 68,8 \\
\hline $24 / 06 / 94$ & 3 & 1 & 6 & 1 & 3 & 0 & 26,4 & 13,4 & 13,0 & 68,8 \\
\hline $26 / 06 / 94$ & 3 & 1 & 5 & 0 & 4 & 0 & 24,4 & 14,9 & 9,5 & 82,1 \\
\hline $30 / 06 / 94$ & 3 & 1 & 5 & 1 & 0 & 0 & 21,4 & 11,2 & 10,2 & 84,4 \\
\hline $01 / 07 / 94$ & 3 & 1 & 4 & 0 & 1 & 0 & 24,5 & 12,1 & 12,4 & 88,0 \\
\hline
\end{tabular}


(continuação...)

Tabela 10: Dados Coletados dos Animais no Período de 31/08/93 a 31/08/94.

\begin{tabular}{|c|c|c|c|c|c|c|c|c|c|c|}
\hline Data & Estaçöes & Atendim. & Idade & Sexo & Diárias & óbito & $\begin{array}{l}\text { Temper. } \\
\text { Máxima }\end{array}$ & $\begin{array}{l}\text { Temper. } \\
\text { Mínima }\end{array}$ & $\begin{array}{l}\text { Aaplitude } \\
\text { Teaper. }\end{array}$ & 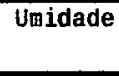 \\
\hline $01 / 07 / 94$ & 3 & 1 & 8 & 1 & 0 & 0 & 24,5 & 12,1 & 12,4 & 88,0 \\
\hline $06 / 07 / 94$ & 3 & 1 & 7 & 1 & 0 & 0 & 22,6 & 7,3 & 15,3 & 53,9 \\
\hline $06 / 07 / 94$ & 3 & 1 & 6 & 1 & 0 & 0 & 22,6 & 7,3 & 15,3 & 53,9 \\
\hline $10 / 07 / 94$ & 3 & 1 & 6 & 0 & 4 & 0 & 21,2 & 16,1 & 5,1 & 88,7 \\
\hline $11 / 07 / 94$ & 3 & 1 & 3 & 1 & 0 & 0 & 24,8 & 14,0 & 10,8 & 71,9 \\
\hline $11 / 07 / 94$ & 3 & 1 & 6 & 1 & 1 & 1 & 24,8 & 14,0 & 10,8 & 71,9 \\
\hline $14 / 07 / 94$ & 3 & 2 & 3 & 1 & 3 & 1 & 24,2 & 15,4 & 8,8 & 83,8 \\
\hline $14 / 07 / 94$ & 3 & 2 & 3 & 1 & 3 & 1 & 24,2 & 15,4 & 8,8 & 83,8 \\
\hline $15 / 07 / 94$ & 3 & 1 & 4 & 1 & 1 & 1 & 17,4 & 7,0 & 10,4 & 62,3 \\
\hline $16 / 07 / 94$ & 3 & 1 & 8 & 0 & 0 & 0 & 12,0 & 0,1 & 11,9 & 57,9 \\
\hline $16 / 07 / 94$ & 3 & 1 & 5 & 1 & 0 & 0 & 12,0 & 0,1 & 11,9 & 57,9 \\
\hline $16 / 07 / 94$ & 3 & 1 & 5 & 1 & 3 & 0 & 12,0 & 0,1 & 11,9 & 57,9 \\
\hline $18 / 07 / 94$ & 3 & 1 & 5 & 1 & 0 & 0 & 18,7 & 5,0 & 13,7 & 66,1 \\
\hline $19 / 07 / 94$ & 3 & 2 & 5 & 0 & 3 & 0 & 21,5 & 9,4 & 12,1 & 59,2 \\
\hline $19 / 07 / 94$ & 3 & 1 & 4 & 0 & 1 & 1 & 21,5 & 9,4 & 12,1 & 59,2 \\
\hline $20 / 07 / 94$ & 3 & 1 & 4 & 1 & 2 & 1 & 23,4 & 7,4 & 16,0 & 60,4 \\
\hline $23 / 07 / 94$ & 3 & 1 & 5 & 0 & 4 & 0 & 26,9 & 11,6 & 15,3 & 47,4 \\
\hline $23 / 07 / 94$ & 3 & 1 & 6 & 0 & 0 & 0 & 26,9 & 11,6 & 15,3 & 47,4 \\
\hline $23 / 07 / 94$ & 3 & 2 & 4 & 0 & 3 & 0 & 26,9 & 11,6 & 15,3 & 47,4 \\
\hline $23 / 07 / 94$ & 3 & 1 & 2 & 1 & 0 & 0 & 26,9 & 11,6 & 15,3 & 47,4 \\
\hline $23 / 07 / 94$ & 3 & 1 & 12 & 0 & 1 & 1 & 26,9 & 11,6 & 15,3 & 47,4 \\
\hline $24 / 07 / 94$ & 3 & 1 & 6 & 0 & 5 & 0 & 28,8 & 9,7 & 19,1 & 53,0 \\
\hline $24 / 07 / 94$ & 3 & 1 & 6 & 0 & 1 & 0 & 28,8 & 9,7 & 19,1 & 53,0 \\
\hline $24 / 07 / 94$ & 3 & 1 & 10 & 1 & 0 & 0 & 28,8 & 9,7 & 19,1 & 53,0 \\
\hline $25 / 07 / 94$ & 3 & 1 & 6 & 0 & 0 & 0 & 28,3 & 13,4 & 14,9 & 50,2 \\
\hline $25 / 07 / 94$ & 3 & 1 & 6 & 1 & 1 & 0 & 28,3 & 13,4 & 14,9 & 50,2 \\
\hline $26 / 07 / 94$ & 3 & 1 & 14 & 0 & 4 & 0 & 29,2 & 13,0 & 16,2 & 46,2 \\
\hline $26 / 07 / 94$ & 3 & 2 & 4 & 0 & 1 & 0 & 29,2 & 13,0 & 16,2 & 46,2 \\
\hline $27 / 07 / 94$ & 3 & 1 & 9 & 1 & 3 & 0 & 28,9 & 12,4 & 16,5 & 44,0 \\
\hline $28 / 07 / 94$ & 3 & 1 & 4 & 0 & 4 & 0 & 29,8 & 11,6 & 18,2 & 43,6 \\
\hline $28 / 07 / 94$ & 3 & 1 & 4 & 1 & 0 & 0 & 29,8 & 11,6 & 18,2 & 43,6 \\
\hline $30 / 07 / 94$ & 3 & 1 & 3 & 0 & 2 & 1 & 17,4 & 10,5 & 6,9 & 77,2 \\
\hline $30 / 07 / 94$ & 3 & 1 & 7 & 0 & 3 & 1 & 17,4 & 10,5 & 6,9 & 77,2 \\
\hline $31 / 07 / 94$ & 3 & 1 & 3 & 0 & 2 & 0 & 19,9 & 6,7 & 13,2 & 71,9 \\
\hline $01 / 08 / 94$ & 3 & 1 & 8 & 0 & 0 & 0 & 25,6 & 8,6 & 17,0 & 70,3 \\
\hline $01 / 08 / 94$ & 3 & 1 & 7 & 1 & 2 & 0 & 25,6 & 8,6 & 17,0 & 70,3 \\
\hline $01 / 08 / 94$ & 3 & 3 & 2 & 1 & 3 & 1 & 25,6 & 8,6 & 17,0 & 70,3 \\
\hline $02 / 08 / 94$ & 3 & 1 & 2 & 1 & 4 & 0 & 23,6 & 13,8 & 9,8 & 81,2 \\
\hline $03 / 08 / 94$ & 3 & 1 & 5 & 1 & 3 & 0 & 29,3 & 15,2 & 14,1 & 56,3 \\
\hline
\end{tabular}

(continua...) 
(continuação...)

Tabela 10: Dados Coletados dos Animais no Período de 31/08/93 a 31/08/94.

\begin{tabular}{|c|c|c|c|c|c|c|c|c|c|c|}
\hline Data & Estações & Atendim. & Idade & Sexo & Diárias & óbito & $\begin{array}{l}\text { Temper. } \\
\text { Máxima }\end{array}$ & $\begin{array}{l}\text { Teaper. } \\
\text { Mínima }\end{array}$ & $\begin{array}{l}\text { Amplitude } \\
\text { Temper. }\end{array}$ & Umidade \\
\hline $03 / 08 / 94$ & 3 & 1 & 5 & 1 & 3 & 0 & 29,3 & 15,2 & 14,1 & 56,3 \\
\hline $04 / 08 / 94$ & 3 & 1 & 4 & 0 & 0 & 0 & 29,9 & 13,4 & 16,5 & 56,2 \\
\hline $04 / 08 / 94$ & 3 & 1 & 4 & 1 & 5 & 0 & 29,9 & 13,4 & 16,5 & 56,2 \\
\hline $04 / 08 / 94$ & 3 & 1 & 5 & 1 & 0 & 0 & 29,9 & 13,4 & 16,5 & 56,2 \\
\hline $06 / 08 / 94$ & 3 & 1 & 9 & 1 & 1 & 0 & 30,0 & 16,0 & 14,0 & 54,2 \\
\hline $07 / 08 / 94$ & 3 & 1 & 3 & 0 & 2 & 0 & 29,6 & 15,2 & 14,4 & 44,6 \\
\hline $07 / 08 / 94$ & 3 & 1 & 4 & 1 & 1 & 1 & 29,6 & 15,2 & 14,4 & 44,6 \\
\hline $09 / 08 / 94$ & 3 & 1 & 48 & 1 & 0 & 0 & 30,1 & 13,2 & 16,9 & 61,1 \\
\hline $09 / 08 / 94$ & 3 & 1 & 2 & 1 & 5 & 1 & 30,1 & 13,2 & 16,9 & 61,1 \\
\hline $10 / 08 / 94$ & 3 & 1 & 5 & 0 & 0 & 0 & 19,4 & 10,2 & 9,2 & 48,6 \\
\hline $10 / 08 / 94$ & 3 & 1 & 10 & 1 & 2 & 1 & 19,4 & 10,2 & 9,2 & 48,6 \\
\hline $11 / 08 / 94$ & 3 & 1 & 14 & 0 & 1 & 0 & 20,3 & 3,8 & 16,5 & 41,9 \\
\hline $11 / 08 / 94$ & 3 & 1 & 2 & 1 & 0 & 0 & 20,3 & 3,8 & 16,5 & 41,9 \\
\hline $11 / 08 / 94$ & 3 & 1 & 3 & 1 & 1 & 0 & 20,3 & 3,8 & 16,5 & 41,9 \\
\hline $11 / 08 / 94$ & 3 & 1 & 2 & 1 & 1 & 0 & 20,3 & 3,8 & 16,5 & 41,9 \\
\hline $12 / 08 / 94$ & 3 & 1 & 5 & 0 & 1 & 0 & 21,0 & 7,7 & 13,3 & 60,5 \\
\hline $12 / 08 / 94$ & 3 & 1 & 2 & 0 & 2 & 1 & 21,0 & 7,7 & 13,3 & 60,5 \\
\hline $12 / 08 / 94$ & 3 & 2 & 19 & 1 & 5 & 1 & 21,0 & 7,7 & 13,3 & 60,5 \\
\hline $13 / 08 / 94$ & 3 & 2 & 2 & 0 & 1 & 0 & 23,4 & 6,2 & 17,2 & 50,6 \\
\hline $15 / 08 / 94$ & 3 & 2 & 6 & 0 & 2 & 0 & 25,0 & 7,9 & 17,1 & 55,5 \\
\hline $15 / 08 / 94$ & 3 & 1 & 7 & 1 & 3 & 0 & 25,0 & 7,9 & 17,1 & 55,5 \\
\hline $15 / 08 / 94$ & 3 & 2 & 6 & 1 & 1 & 1 & 25,0 & 7,9 & 17,1 & 55,5 \\
\hline $16 / 08 / 94$ & 3 & 1 & 24 & 1 & 0 & 1 & 29,4 & 12,8 & 16,6 & 39,5 \\
\hline $17 / 08 / 94$ & 3 & 2 & 6 & 1 & 3 & 0 & 24,0 & 14,8 & 9,2 & 72,5 \\
\hline $17 / 08 / 94$ & 3 & 1 & 3 & 0 & 0 & 1 & 24,0 & 14,8 & 9,2 & 72,5 \\
\hline $18 / 08 / 94$ & 3 & 2 & 13 & 0 & 3 & 0 & 23,4 & 8,3 & 15,1 & 62,0 \\
\hline $18 / 08 / 94$ & 3 & 2 & 5 & 1 & 3 & 0 & 23,4 & 8,3 & 15,1 & 62,0 \\
\hline $19 / 08 / 94$ & 3 & 1 & 6 & 1 & 6 & $\mathbf{o}$ & 21,6 & 9,6 & 12,0 & 64,8 \\
\hline $20 / 08 / 94$ & 3 & 1 & 18 & 1 & 4 & $\mathbf{0}$ & 26,8 & 11,8 & 15,0 & 66,3 \\
\hline $20 / 08 / 94$ & 3 & 1 & 4 & 1 & 4 & 0 & 26,8 & 11,8 & 15,0 & 66,3 \\
\hline $20 / 08 / 94$ & 3 & 1 & 8 & 1 & 2 & 1 & 26,8 & 11,8 & 15,0 & 66,3 \\
\hline $21 / 08 / 94$ & 3 & 1 & 46 & 0 & 0 & 0 & 28,0 & 14,2 & 13,8 & 52,4 \\
\hline $21 / 08 / 94$ & 3 & 1 & 9 & 1 & 1 & 0 & 28,0 & 14,2 & 13,8 & 52,4 \\
\hline $21 / 08 / 94$ & 3 & 1 & 24 & 0 & 0 & 1 & 28,0 & 14,2 & 13,8 & 52,4 \\
\hline $22 / 08 / 94$ & 3 & 1 & 3 & 1 & 5 & $\mathbf{0}$ & 28,2 & 13,8 & 14,4 & 47,5 \\
\hline $22 / 08 / 94$ & 3 & 1 & 5 & 1 & 1 & 1 & 28,2 & 13,8 & 14,4 & 47,5 \\
\hline $23 / 08 / 94$ & 3 & 1 & 5 & 1 & 5 & 0 & 30,2 & 14,4 & 15,8 & 51,6 \\
\hline $23 / 08 / 94$ & 3 & 2 & 4 & 1 & 8 & 0 & 30,2 & 14,4 & 15,8 & 51,6 \\
\hline $24 / 08 / 94$ & 3 & 1 & 2 & 1 & 1 & 1 & 24,8 & 14,8 & 10,0 & 65,8 \\
\hline
\end{tabular}


(continuação...)

Tabela 10: Dados Coletados dos Animais no Período de 31/08/93 a 31/08/94.

\begin{tabular}{cccccccccccc}
\hline Data & Estações Atendim. & Idade & Sexo & Diárias & obito & $\begin{array}{c}\text { Temper. } \\
\text { Máxima }\end{array}$ & $\begin{array}{c}\text { Temper. } \\
\text { Mínima }\end{array}$ & $\begin{array}{c}\text { Amplitude } \\
\text { Temper. }\end{array}$ & Umidade \\
\hline $25 / 08 / 94$ & 3 & 1 & 5 & 1 & 3 & 0 & 25,6 & 10,3 & 15,3 & 62,7 \\
$26 / 08 / 94$ & 3 & 1 & 3 & 0 & 0 & 0 & 27,8 & 13,4 & 14,4 & 56,4 \\
$26 / 08 / 94$ & 3 & 1 & 4 & 0 & 1 & 1 & 27,8 & 13,4 & 14,4 & 56,4 \\
$26 / 08 / 94$ & 3 & 1 & 7 & 1 & 3 & 1 & 27,8 & 13,4 & 14,4 & 56,4 \\
$27 / 08 / 94$ & 3 & 1 & 3 & 0 & 0 & 0 & 29,8 & 14,2 & 15,6 & 41,9 \\
$27 / 08 / 94$ & 3 & 1 & 3 & 1 & 0 & 0 & 29,8 & 14,2 & 15,6 & 41,9 \\
$29 / 08 / 94$ & 3 & 1 & 18 & 0 & 0 & 0 & 31,6 & 14,2 & 17,4 & 39,2 \\
$30 / 08 / 94$ & 3 & 1 & 18 & 0 & 6 & 0 & 31,8 & 15,6 & 16,2 & 41,8 \\
$30 / 08 / 94$ & 3 & 1 & 4 & 0 & 3 & 0 & 31,8 & 15,6 & 16,2 & 41,8 \\
$30 / 08 / 94$ & 3 & 3 & 2 & 1 & 2 & 0 & 31,8 & 15,6 & 16,2 & 41,8 \\
$30 / 08 / 94$ & 3 & 1 & 4 & 1 & 2 & 1 & 31,8 & 15,6 & 16,2 & 41,8 \\
$30 / 08 / 94$ & 3 & 1 & 4 & 1 & 1 & 1 & 31,8 & 15,6 & 16,2 & 41,8 \\
$30 / 08 / 94$ & 3 & 2 & 5 & 1 & 2 & 1 & 31,8 & 15,6 & 16,2 & 41,8 \\
$31 / 08 / 94$ & 3 & 1 & 3 & 1 & 2 & 0 & 26,0 & 14,4 & 11,6 & 61,3 \\
$31 / 08 / 94$ & 3 & 1 & 2 & 1 & 1 & 1 & 26,0 & 14,4 & 11,6 & 61,3 \\
$31 / 08 / 94$ & 3 & 1 & 2 & 1 & 2 & 1 & 26,0 & 14,4 & 11,6 & 61,3 \\
\hline
\end{tabular}


Tabela 11: Análise de Resíduos do Conjunto de Dados

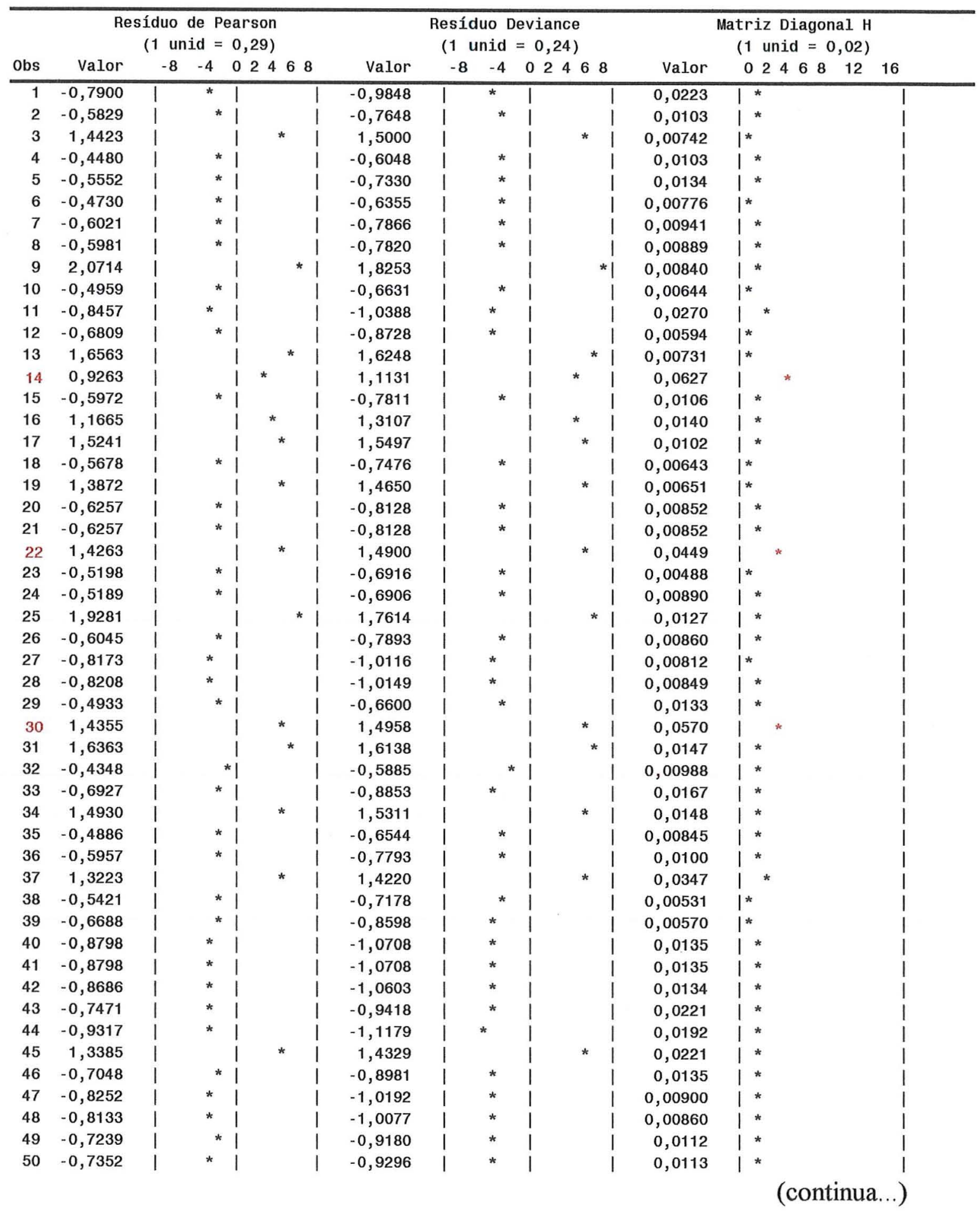


(continuação...)

Tabela 11: Análise de Resíduos do Conjunto de dados

\begin{tabular}{|c|c|c|c|c|c|c|c|c|c|c|c|c|c|c|c|}
\hline \multirow[b]{2}{*}{ Obs } & \multicolumn{6}{|c|}{$\begin{array}{l}\text { Resíduo de Pearson } \\
(1 \text { unid }=0,29)\end{array}$} & \multicolumn{4}{|c|}{$\begin{array}{l}\text { Resíduo Deviance } \\
(1 \text { unid }=0,24)\end{array}$} & \multicolumn{5}{|c|}{$\begin{array}{l}\text { Matriz Diagonal } \mathrm{H} \\
(1 \text { unid }=0,02)\end{array}$} \\
\hline & Valor & -8 & $\begin{array}{ll}-4 & 0\end{array}$ & 246 & 8 & Valor & -8 & -4 & 024 & 68 & Valor & 0246 & 8 & 12 & 16 \\
\hline 51 & $-0,7226$ & $\mathrm{~T}$ & * I & & T & $-0,9166$ & I & * & $T$ & $T$ & 0,0104 & $T^{*}$ & & & 1 \\
\hline 52 & 1,3839 & i & i & * & i & 1,4629 & i & & i & * i & 0,0104 & i* & & & i \\
\hline 53 & $-0,5677$ & i & * 1 & & i & $-0,7475$ & i & * & i & i & 0,00820 & i * & & & i \\
\hline 54 & $-0,8033$ & I & * $\quad 1$ & & i & $-0,9979$ & 1 & * & I & i & 0,0653 & i & & & i \\
\hline 55 & $-0,7270$ & i & * $\quad 1$ & & i & $-0,9212$ & i & * & i & i & 0,00836 & 1* & & & i \\
\hline 56 & $-0,8384$ & i & * i & & i & $-1,0318$ & i & * & i & i & 0,0214 & 1 * & & & i \\
\hline 57 & $-0,6842$ & i & * 1 & & 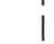 & $-0,8763$ & 1 & * & 1 & 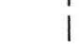 & 0,1023 & 1 & & & i \\
\hline 58 & $-0,3964$ & i & $* 1$ & & I & $-0,5403$ & I & * & i & 1 & 0,0710 & * & & & I \\
\hline 59 & 1,6981 & 1 & 1 & * & I & 1,6473 & | & & I & * 1 & 0,0270 & 1 * & & & I \\
\hline 60 & $-0,6845$ & i & $* 1$ & & i & $-0,8766$ & i & * & i & i & 0,00804 & |* & & & i \\
\hline 61 & 1,3344 & i & i & * & i & 1,4302 & i & & i & * i & 0,00665 & |* & & & i \\
\hline 62 & $-0,2326$ & i & $* 1$ & & 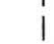 & $-0,3246$ & i & & *1 & | & 0,2002 & 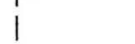 & & * & i \\
\hline 63 & $-0,6360$ & i & $* i$ & & 1 & $-0,8243$ & i & * & i & 1 & 0,00633 & * & & & i \\
\hline 64 & 1,5722 & I & i & * & I & 1,5778 & 1 & & i & * 1 & 0,00633 & * & & & I \\
\hline 65 & 1,5194 & i & i & * & i & 1,5470 & i & & i & * i & 0,00796 & |* & & & i \\
\hline 66 & $-0,7566$ & i & * 1 & & i & $-0,9514$ & i & * & i & i & 0,1280 & $i$ & * & & i \\
\hline 67 & 1,3831 & i & i & * & I & 1,4623 & i & & i & * 1 & 0,00541 & $1^{*}$ & & & i \\
\hline 68 & $-0,8197$ & i & $* \quad 1$ & & i & $-1,0139$ & i & * & $i$ & i & 0,00948 & | * & & & i \\
\hline 69 & $-0,4707$ & I & $* 1$ & & I & $-0,6326$ & i & * & I & 1 & 0,00818 & * & & & i \\
\hline 70 & $-0,5412$ & | & $*$ & & 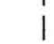 & $-0,7167$ & 1 & * & i & i & 0,0114 & * & & & i \\
\hline 71 & $-0,5831$ & i & $* 1$ & & i & $-0,7650$ & i & * & i & i & 0,00923 & i * & & & i \\
\hline 72 & $-0,5861$ & i & $* i$ & & i & $-0,7685$ & $i$ & * & i & i & 0,00910 & | * & & & i \\
\hline 73 & 2,0806 & 1 & i & & * & 1,8293 & 1 & & i & *1 & 0,00795 & |* & & & I \\
\hline 74 & 1,7240 & i & I & * & $* 1$ & 1,6609 & 1 & & i & * I & 0,00940 & * & & & I \\
\hline 75 & 1,2612 & 1 & 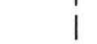 & * & i & 1,3798 & 1 & & i & * & 0,0311 & * & & & I \\
\hline 76 & 1,4060 & i & i & * & I & 1,4771 & I & & i & * I & 0,0374 & * & & & I \\
\hline 77 & $-0,5812$ & i & $* 1$ & & I & $-0,7629$ & i & * & i & i & 0,0104 & * & & & i \\
\hline 78 & $-0,6954$ & I & * & & 1 & $-0,8882$ & 1 & * & I & i & 0,0105 & 1 * & & & i \\
\hline 79 & $-0,5315$ & i & $* 1$ & & I & $-0,7054$ & 1 & * & 1 & 1 & 0,0578 & 1 & & & i \\
\hline 80 & $-0,5742$ & i & $* 1$ & & 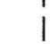 & $-0,7549$ & 1 & * & 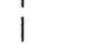 & 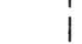 & 0,0197 & * & & & i \\
\hline 81 & 1,2537 & i & $i$ & $*$ & i & 1,3745 & i & & i & * 1 & 0,0262 & 1 * & & & i \\
\hline 82 & 1,1277 & i & i & * & 1 & 1,2810 & i & & i & * $\quad 1$ & 0,0179 & * & & & i \\
\hline 83 & $-0,8064$ & i & * 1 & & I & $-1,0010$ & i & * & i & I & 0,0304 & 1 * & & & I \\
\hline 84 & 1,1716 & i & i & $*$ & I & 1,3145 & 1 & & i & * $\quad 1$ & 0,0333 & * & & & I \\
\hline 85 & 1,4892 & i & i & * & 1 & 1,5288 & 1 & & i & * & 0,0385 & * & & & I \\
\hline 86 & 0,9457 & 1 & i & $*$ & i & 1,1304 & i & & i & * I & 0,0328 & 1 * & & & i \\
\hline 87 & $-0,5669$ & i & $* 1$ & & i & $-0,7466$ & i & $*$ & i & i & 0,0204 & 1 * & & & i \\
\hline 88 & $-0,8451$ & i & * 1 & & I & $-1,0382$ & I & $*$ & i & I & 0,0175 & | * & & & I \\
\hline 89 & 1,7527 & i & i & * & I & 1,6758 & i & & I & * I & 0,0420 & | * & & & i \\
\hline 90 & $-0,6417$ & | & $* 1$ & & I & $-0,8305$ & 1 & * & I & I & 0,0168 & * & & & I \\
\hline 91 & $-0,8533$ & i & * i & & I & $-1,0460$ & i & $*$ & i & i & 0,0133 & 1 * & & & i \\
\hline 92 & $-0,8597$ & i & * 1 & & i & $-1,0520$ & i & * & i & 1 & 0,0136 & |* & & & i \\
\hline 93 & 1,4662 & 1 & i & * & i & 1,5148 & 1 & & i & * 1 & 0,0132 & * & & & I \\
\hline 94 & $-0,5788$ & $i$ & $* 1$ & & i & $-0,7601$ & | & $*$ & i & I & 0,00665 & * & & & i \\
\hline 95 & $-0,6080$ & 1 & $* 1$ & & i & $-0,7932$ & | & $*$ & 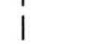 & i & 0,00669 & * & & & I \\
\hline 96 & $-0,5539$ & i & $* 1$ & & i & $-0,7316$ & i & * & i & i & 0,0173 & | * & & & i \\
\hline 97 & $-0,6719$ & 1 & $* 1$ & & 1 & $-0,8632$ & 1 & $*$ & i & 1 & 0,00754 & |* & & & I \\
\hline 98 & $-0,7205$ & 1 & $* 1$ & & i & $-0,9145$ & 1 & $*$ & 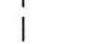 & 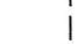 & 0,0181 & * & & & I \\
\hline 99 & $-0,4973$ & 1 & $* 1$ & & I & $-0,6648$ & | & $*$ & | & i & 0,0187 & $1 *$ & & & i \\
\hline 100 & $-0,5629$ & 1 & $* 1$ & & 1 & $-0,7419$ & 1 & * & i & I & 0,00464 & |* & & & i \\
\hline 101 & $-0,7092$ & 1 & $* 1$ & & I & $-0,9027$ & I & $*$ & | & I & 0,00414 & * & & & i \\
\hline 102 & 1,3865 & i & i & $*$ & i & 1,4645 & I & & I & * I & 0,00442 & |* & & & i \\
\hline
\end{tabular}


(continuação...)

Tabela 11: Análise de Resíduos do Conjunto de dados

\begin{tabular}{|c|c|c|c|c|c|c|c|c|c|c|c|c|c|c|}
\hline \multirow[b]{2}{*}{ Obs } & \multicolumn{5}{|c|}{$\begin{array}{l}\text { Resíduo de Pearson } \\
(1 \text { unid }=0,29)\end{array}$} & \multicolumn{5}{|c|}{$\begin{array}{l}\text { Resíduo Deviance } \\
\text { (1 unid }=0,24)\end{array}$} & \multicolumn{4}{|c|}{$\begin{array}{l}\text { Matriz Diagonal } \mathrm{H} \\
(1 \text { unid }=0,02)\end{array}$} \\
\hline & Valor & -8 & $\begin{array}{lll}-4 & 0 & 2\end{array}$ & 468 & & Valor & -8 & -4 & 024 & 68 & Valor & 0246 & 81 & 16 \\
\hline 103 & $-0,5661$ & I & $* 1$ & & $\mathrm{~T}$ & $-0,7456$ & $\mathrm{I}$ & $*$ & $\mathrm{~T}$ & 1 & 0,0114 & $1 *$ & & $\overline{1}$ \\
\hline 104 & $-0,6334$ & I & $* 1$ & & i & $-0,8214$ & i & * & I & I & 0,00822 & $1 *$ & & i \\
\hline 105 & $-0,7795$ & i & $* 1$ & & I & $-0,9744$ & i & * & i & i & 0,00773 & i* & & i \\
\hline 106 & $-0,5590$ & i & $* i$ & & i & $-0,7375$ & i & * & i & I & 0,0106 & 1 * & & I \\
\hline 107 & 1,7524 & i & i & * & 1 & 1,6756 & i & & i & * 1 & 0,0113 & * & & i \\
\hline 108 & $-0,6468$ & I & $* 1$ & & i & $-0,8360$ & i & $*$ & i & i & 0,00734 & $1^{*}$ & & i \\
\hline 109 & $-0,4975$ & i & $* i$ & & i & $-0,6650$ & i & * & i & i & 0,00769 & |* & & i \\
\hline 110 & $-0,6202$ & i & $* i$ & & i & $-0,8067$ & i & * & i & i & 0,00660 & |* & & i \\
\hline 111 & $-0,6182$ & i & $* 1$ & & i & $-0,8046$ & i & * & i & i & 0,00718 & |* & & I \\
\hline 112 & $-0,6894$ & I & $* 1$ & & i & $-0,8818$ & i & $*$ & i & I & 0,00824 & $1 *$ & & i \\
\hline 113 & $-0,5447$ & i & $* i$ & & i & $-0,7209$ & i & * & i & i & 0,00937 & 1* & & i \\
\hline 114 & $-0,5447$ & I & $* 1$ & & i & $-0,7209$ & I & * & I & I & 0,00937 & $1 *$ & & i \\
\hline 115 & $-0,6475$ & 1 & $* 1$ & & i & $-0,8368$ & i & * & i & i & 0,0117 & 1* & & i \\
\hline 116 & 1,3191 & I & I & * & I & 1,4198 & i & & i & * i & 0,0210 & $i *$ & & i \\
\hline 117 & $-0,6110$ & i & $* i$ & & i & $-0,7965$ & i & $*$ & I & i & 0,0143 & | * & & I \\
\hline 118 & $-1,5703$ & i & $i$ & & i & $-1,5767$ & $1 *$ & & i & i & 0,1245 & i & * & I \\
\hline 119 & $-0,7147$ & 1 & $* 1$ & & I & $-0,9085$ & 1 & * & i & 1 & 0,0137 & 1 * & & i \\
\hline 120 & 1,7332 & I & i & * & i & 1,6657 & i & & i & * i & 0,0119 & 1 * & & i \\
\hline 121 & $-0,6336$ & i & $* 1$ & & i & $-0,8216$ & i & * & I & 1 & 0,0153 & |* & & I \\
\hline 122 & $-0,9864$ & i & * 1 & & I & $-1,1658$ & i & * & i & i & 0,0521 & $1=$ & & i \\
\hline 123 & $-0,8205$ & i & * 1 & & i & $-1,0147$ & i & * & i & i & 0,0137 & 1* & & i \\
\hline 124 & $-0,7368$ & I & $* 1$ & & i & $-0,9313$ & i & * & i & I & 0,0677 & 1 & & i \\
\hline 125 & 1,5097 & i & i & $*$ & 1 & 1,5412 & i & & i & * i & 0,0124 & $1 *$ & & i \\
\hline 126 & 1,6133 & i & i & * & i & 1,6011 & i & & i & * & 0,0140 & 1 * & & i \\
\hline 127 & 1,2598 & i & 1 & * & i & 1,3788 & i & & i & * & 0,0144 & 1 * & & i \\
\hline 128 & $-0,7487$ & i & $* i$ & & i & $-0,9434$ & i & * & i & I & 0,00781 & |* & & I \\
\hline 129 & 1,7067 & i & 1 & * & i & 1,6518 & i & & i & * & 0,00649 & $1^{*}$ & & I \\
\hline 130 & 1,1881 & i & i & $*$ & i & 1,3268 & i & & i & * i & 0,0801 & 1 & & i \\
\hline 131 & $-0,4998$ & i & $* 1$ & & i & $-0,6678$ & i & * & i & I & 0,00767 & * & & I \\
\hline 132 & $-0,6463$ & i & $* i$ & & i & $-0,8356$ & i & * & $i$ & i & 0,0101 & 1* & & I \\
\hline 133 & $-0,5873$ & i & $* 1$ & & i & $-0,7699$ & i & * & i & i & 0,0110 & I * & & I \\
\hline 134 & $-0,7272$ & i & * i & & i & $-0,9214$ & i & $*$ & I & I & 0,0120 & 1 * & & I \\
\hline 135 & $-0,5873$ & $i$ & $* 1$ & & i & $-0,7699$ & i & $*$ & i & i & 0,0110 & |* & & i \\
\hline 136 & $-0,7102$ & i & $* 1$ & & i & $-0,9038$ & i & $*$ & i & 1 & 0,0110 & * & & I \\
\hline 137 & 1,3744 & i & i & * & i & 1,4567 & i & & i & * 1 & 0,0119 & * & & i \\
\hline 138 & 1,3689 & I & i & * & i & 1,4531 & i & & I & * 1 & 0,0109 & * & & i \\
\hline 139 & $-0,5855$ & i & $* 1$ & & I & $-0,7678$ & I & * & 1 & I & 0,00481 & $1^{*}$ & & i \\
\hline 140 & $-0,5824$ & i & $* 1$ & & i & $-0,7643$ & i & * & i & i & 0,00495 & $1^{*}$ & & i \\
\hline 141 & $-0,5695$ & i & $* 1$ & & i & $-0,7495$ & i & * & I & I & 0,00568 & $1^{*}$ & & I \\
\hline 142 & $-0,5695$ & i & $* 1$ & & i & $-0,7495$ & 1 & * & i & i & 0,00568 & * & & I \\
\hline 143 & $-0,6991$ & i & $* 1$ & & I & $-0,8921$ & i & * & 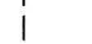 & I & 0,00581 & * & & i \\
\hline 144 & 1,3861 & $i$ & i & * & i & 1,4642 & i & & i & * & 0,00496 & * & & i \\
\hline 145 & 1,3861 & i & I & * & I & 1,4642 & 1 & & 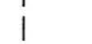 & * 1 & 0,00496 & * & & i \\
\hline 146 & 1,5690 & i & i & * & i & 1,5759 & i & & i & $* 1$ & 0,00736 & $1^{*}$ & & i \\
\hline 147 & $-0,5912$ & i & $* 1$ & & | & $-0,7743$ & i & * & i & I & 0,00984 & * & & I \\
\hline 148 & $-0,5413$ & i & $* 1$ & & I & $-0,7169$ & i & $*$ & 1 & I & 0,0100 & 1 * & & I \\
\hline 149 & $-0,7212$ & i & $* 1$ & & i & $-0,9152$ & 1 & $*$ & 1 & 1 & 0,0132 & * & & I \\
\hline 150 & 1,4116 & I & I & * & I & 1,4806 & i & & i & * 1 & 0,0127 & * & & i \\
\hline 151 & $-0,6236$ & i & $* 1$ & & i & $-0,8105$ & i & * & i & i & 0,00608 & |* & & i \\
\hline 152 & 1,6067 & i & i & * & | & 1,5974 & i & & i & * & 0,00600 & * & & i \\
\hline 153 & $-0,5620$ & i & $* 1$ & & i & $-0,7409$ & i & $*$ & 1 & 1 & 0,00553 & * & & I \\
\hline 154 & $-0,6352$ & i & $* 1$ & & I & $-0,8233$ & i & $*$ & $i$ & I & 0,00648 & * & & I \\
\hline
\end{tabular}


(continuação...)

Tabela 11: Análise de Resíduos do Conjunto de dados

\begin{tabular}{|c|c|c|c|c|c|c|c|c|c|c|c|c|c|c|c|c|}
\hline \multirow[b]{2}{*}{ Obs } & \multicolumn{7}{|c|}{$\begin{array}{l}\text { Resíduo de Pearson } \\
\quad(1 \text { unid }=0,29)\end{array}$} & \multicolumn{4}{|c|}{$\begin{array}{l}\text { Resíduo Deviance } \\
(1 \text { unid }=0,24)\end{array}$} & \multicolumn{5}{|c|}{$\begin{array}{l}\text { Matriz Diagonal } \mathrm{H} \\
(1 \text { unid }=0,02)\end{array}$} \\
\hline & Valor & -8 & -4 & 02 & 4 & 68 & Valor & -8 & -4 & 024 & 468 & Valor & 02 & 468 & 12 & 16 \\
\hline 155 & 1,8029 & 1 & & 1 & & 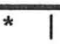 & 1,7012 & $\mathrm{~T}$ & & 1 & $* 1$ & 0,00542 & $1^{*}$ & & & \\
\hline 156 & $-0,6082$ & I & $*$ & 1 & & I & $-0,7934$ & I & * & I & I & 0,00740 & |* & & & \\
\hline 157 & 1,0130 & I & & I & * & I & 1,1884 & I & & I & 1 & 0,1485 & I & & * & \\
\hline 158 & $-0,7508$ & I & * & I & & i & $-0,9456$ & i & * & i & i & 0,00525 & |* & & & \\
\hline 159 & 1,3254 & I & & i & * & i & 1,4241 & i & & i & * i & 0,00510 & I* & & & \\
\hline 160 & $-0,5374$ & I & * & I & & I & $-0,7124$ & 1 & * & 1 & I & 0,00447 & $I^{*}$ & & & \\
\hline 161 & $-0,6365$ & i & * & i & & i & $-0,8248$ & i & * & i & i & 0,0159 & i * & & & \\
\hline 162 & $-0,5312$ & I & * & i & & i & $-0,7051$ & i & * & 1 & i & 0,00447 & $\left.\right|^{*}$ & & & \\
\hline 163 & $-0,7374$ & 1 & $*$ & i & & i & $-0,9319$ & i & $*$ & i & i & 0,00681 & |* & & & \\
\hline 164 & $-0,6776$ & i & * & i & & i & $-0,8693$ & i & * & i & i & 0,00445 & |* & & & \\
\hline 165 & $-0,6776$ & 1 & * & I & & 1 & $-0,8693$ & 1 & * & I & I & 0,00445 & I* & & & \\
\hline 166 & 2,0204 & i & & i & & * 1 & 1,8031 & i & & i & $* i$ & 0,00623 & |* & & & \\
\hline 167 & $-0,5380$ & i & * & i & & I & $-0,7130$ & i & * & i & i & 0,00777 & |* & & & \\
\hline 168 & $-0,6782$ & i & * & i & & i & $-0,8700$ & i & $*$ & i & i & 0,00838 & i * & & & \\
\hline 169 & $-0,6665$ & i & * & i & & i & $-0,8574$ & i & * & i & I & 0,00874 & * & & & \\
\hline 170 & $-0,5986$ & i & * & i & & i & $-0,7826$ & i & * & i & i & 0,0151 & I * & & & \\
\hline 171 & 1,2341 & i & & i & * & i & 1,3605 & i & & i & $* 1$ & 0,0181 & i * & & & \\
\hline 172 & $-0,5417$ & i & * & i & & i & $-0,7173$ & i & * & i & i & 0,00930 & 1 * & & & \\
\hline 173 & $-0,7202$ & I & * & I & & I & $-0,9142$ & I & * & I & i & 0,00702 & $1^{*}$ & & & \\
\hline 174 & $-0,7130$ & i & * & i & & i & $-0,9066$ & i & * & i & i & 0,00952 & 1 * & & & \\
\hline 175 & 1,3607 & i & & i & * & i & 1,4477 & i & & $i$ & * 1 & 0,00675 & |* & & & \\
\hline 176 & $-0,6363$ & i & * & i & & i & $-0,8246$ & i & * & i & i & 0,0145 & i* & & & \\
\hline 177 & $-0,5718$ & i & * & i & & i & $-0,7522$ & i & * & i & i & 0,00548 & i* & & & \\
\hline 178 & $-0,7084$ & I & * & & & i & $-0,9018$ & i & * & i & i & 0,00572 & |* & & & \\
\hline 179 & $-0,7415$ & i & & i & & i & $-0,9361$ & i & * & i & i & 0,00669 & |* & & & \\
\hline 180 & $-0,6540$ & I & * & I & & I & $-0,8439$ & I & * & I & i & 0,00695 & I* & & & \\
\hline 181 & 1,7892 & i & & i & & * $\quad 1$ & 1,6943 & i & & i & $* 1$ & 0,00550 & * & & & \\
\hline 182 & 1,4946 & i & & i & * & 1 & 1,5321 & i & & i & * i & 0,00625 & |* & & & \\
\hline 183 & 1,8265 & 1 & & i & & * 1 & 1,7129 & i & & i & $* i$ & 0,00436 & |* & & & \\
\hline 184 & 1,4956 & i & & i & * & $=1$ & 1,5327 & i & & i & * i & 0,00449 & * & & & \\
\hline 185 & 1,5028 & 1 & & I & * & I & 1,5370 & I & & i & * $\quad 1$ & 0,00464 & |* & & & \\
\hline 186 & $-0,7710$ & i & * & i & & i & $-0,9659$ & i & * & i & I & 0,0186 & * & & & \\
\hline 187 & $-0,7522$ & i & & i & & i & $-0,9470$ & i & * & i & i & 0,0188 & 1 * & & & \\
\hline 188 & $-0,6780$ & i & * & i & & i & $-0,8698$ & i & * & i & i & 0,0106 & i * & & & \\
\hline 189 & 2,0444 & i & & i & & * & 1,8137 & $i$ & & i & $* 1$ & 0,00819 & * & & & \\
\hline 190 & $-0,6964$ & i & * & i & & i & $-0,8893$ & i & * & i & I & 0,0105 & |* & & & \\
\hline 191 & 1,6081 & i & & i & & i & 1,5982 & i & & i & $* 1$ & 0,00845 & 1 * & & & \\
\hline 192 & 1,2665 & i & & i & * & i & 1,3835 & i & & i & * $\quad 1$ & 0,00994 & i * & & & \\
\hline 193 & $-0,5637$ & i & * & & & i & $-0,7428$ & i & * & i & $i$ & 0,0121 & i * & & & \\
\hline 194 & $-0,4681$ & i & * & i & & i & $-0,6295$ & i & * & i & i & 0,00857 & i * & & & \\
\hline 195 & $-0,5799$ & I & * & I & & i & $-0,7615$ & i & * & i & i & 0,00931 & i * & & & \\
\hline 196 & $-0,5765$ & i & * & i & & i & $-0,7576$ & i & * & i & i & 0,00907 & * & & & \\
\hline 197 & $-0,5005$ & i & * & i & & i & $-0,6687$ & i & * & $i$ & i & 0,00625 & |* & & & \\
\hline 198 & $-0,6238$ & I & * & & & i & $-0,8107$ & i & * & i & i & 0,00630 & |* & & & \\
\hline 199 & $-0,9251$ & 1 & $*$ & i & & I & $-1,1120$ & i & * & i & $i$ & 0,0473 & i & * & & \\
\hline 200 & 1, 3391 & I & & I & * & I & 1,4333 & i & & i & * i & 0,0448 & i & * & & \\
\hline 201 & 1,0714 & i & & i & $*$ & i & 1,2366 & i & & i & * $\quad 1$ & 0,1503 & 1 & & * & \\
\hline 202 & $-0,5534$ & i & * & i & & i & $-0,7310$ & i & * & i & i & 0,00535 & |* & & & \\
\hline 203 & $-0,8879$ & I & * & i & & i & $-1,0782$ & i & * & i & i & 0,0566 & $i$ & * & & \\
\hline 204 & 1,2140 & 1 & & i & * & I & 1,3459 & i & & i & * i & 0,0318 & $1 *$ & & & \\
\hline 205 & $-0,6543$ & 1 & * & I & & I & $-0,8442$ & I & * & i & I & 0,00544 & $1^{*}$ & & & \\
\hline 206 & $-0,7288$ & i & $*$ & i & & i & $-0,9231$ & i & * & i & i & 0,00574 & |* & & & \\
\hline
\end{tabular}


(continuação...)

Tabela 11: Análise de Resíduos do Conjunto de dados

\begin{tabular}{|c|c|c|c|c|c|c|c|c|c|c|c|c|c|c|c|c|c|}
\hline \multirow[b]{2}{*}{ Obs } & \multicolumn{6}{|c|}{$\begin{array}{c}\text { Resíduo de Pearson } \\
\text { (1 unid }=0,29)\end{array}$} & \multicolumn{6}{|c|}{$\begin{array}{l}\text { Resíduo Deviance } \\
(1 \text { unid }=0,24)\end{array}$} & \multicolumn{5}{|c|}{$\begin{array}{l}\text { Matriz Diagonal } \mathrm{H} \\
(1 \text { unid }=0,02)\end{array}$} \\
\hline & Valor & -8 & -4 & 02 & 46 & 8 & Valor & -8 & -4 & 02 & 68 & & Valor & 02 & 468 & 12 & 16 \\
\hline 207 & $-0,6579$ & 1 & * & & & $T$ & $-0,8481$ & 1 & * & $T$ & & $T$ & 0,00528 & 1* & & & $\bar{T}$ \\
\hline 208 & 1,4965 & i & & i & * & i & 1,5333 & i & & i & $*$ & I & 0,00495 & 1* & & & i \\
\hline 209 & $-0,5542$ & I & * & I & & 1 & $-0,7319$ & 1 & * & I & & 1 & 0,00980 & 1 * & & & I \\
\hline 210 & $-0,6141$ & I & * & I & & I & $-0,8000$ & I & * & I & & 1 & 0,00835 & I * & & & I \\
\hline 211 & 1,7547 & i & & i & * & i & 1,6768 & i & & i & * & $i$ & 0,00904 & | * & & & i \\
\hline 212 & $-0,6055$ & I & * & & & I & $-0,7903$ & I & * & i & & I & 0,0166 & $1 *$ & & & i \\
\hline 213 & 1,2530 & i & & i & * & i & 1,3739 & i & & i & * & i & 0,0200 & | * & & & i \\
\hline 214 & 1,2925 & I & & I & * & i & 1,4016 & i & & i & $*$ & i & 0,0194 & | * & & & i \\
\hline 215 & $-0,8756$ & I & $*$ & 1 & & 1 & $-1,0669$ & I & * & I & & i & 0,0440 & 1 & * & & i \\
\hline 216 & 1,4189 & I & & I & * & i & 1,4853 & I & & I & * & 1 & 0,0168 & $1 *$ & & & i \\
\hline 217 & $-0,5083$ & i & * & i & & i & $-0,6780$ & i & * & i & & i & 0,0131 & $1 *$ & & & i \\
\hline 218 & $-0,5224$ & i & * & i & & I & $-0,6947$ & I & * & i & & i & 0,0126 & | * & & & I \\
\hline 219 & $-0,5142$ & 1 & * & I & & I & $-0,6850$ & I & * & i & & i & 0,0185 & $1 *$ & & & i \\
\hline 220 & $-0,7516$ & i & $*$ & i & & i & $-0,9464$ & i & $*$ & i & & i & 0,0196 & |* & & & i \\
\hline 221 & 1,7036 & i & & i & * & i & 1,6502 & i & & i & * & I & 0,0164 & $1 *$ & & & I \\
\hline 222 & $-0,6557$ & I & * & & & I & $-0,8458$ & i & $*$ & i & & i & 0,00967 & 1* & & & i \\
\hline 223 & $-0,5986$ & i & * & i & & i & $-0,7826$ & i & * & i & & i & 0,00968 & | * & & & i \\
\hline 224 & $-0,5174$ & i & * & i & & i & $-0,6887$ & i & * & I & & i & 0,00762 & $1^{*}$ & & & i \\
\hline 225 & $-0,7814$ & i & & i & & i & $-0,9763$ & i & $*$ & i & & i & 0,0591 & i & * & & i \\
\hline 226 & $-0,5530$ & i & * & $i$ & & i & $-0,7305$ & i & * & I & & i & 0,0121 & * & & & i \\
\hline 227 & $-0,5013$ & i & * & i & & i & $-0,6696$ & i & $*$ & I & & 1 & 0,00680 & * & & & i \\
\hline 228 & $-0,4836$ & | & * & i & & i & $-0,6484$ & i & * & i & & i & 0,00745 & * & & & i \\
\hline 229 & $-0,6143$ & i & * & i & & i & $-0,8002$ & i & * & I & & I & 0,00762 & |* & & & i \\
\hline 230 & $-0,6077$ & i & * & i & & i & $-0,7928$ & i & $*$ & i & & I & 0,00785 & |* & & & i \\
\hline 231 & $-0,6416$ & i & * & i & & I & $-0,8304$ & I & * & I & & I & 0,00738 & | * & & & i \\
\hline 232 & 1,5585 & i & & i & * & i & 1,5699 & i & & i & * & i & 0,00738 & |* & & & i \\
\hline 233 & $-0,9239$ & i & & i & & i & $-1,1110$ & I & * & I & & I & 0,1425 & 1 & & * & I \\
\hline 234 & $-0,6631$ & i & * & i & & i & $-0,8538$ & i & * & i & & i & 0,00877 & i* & & & i \\
\hline 235 & $-0,6836$ & i & * & $i$ & & i & $-0,8757$ & i & $*$ & i & & i & 0,00909 & * & & & i \\
\hline 236 & $-0,5791$ & i & * & i & & i & $-0,7605$ & 1 & * & i & & | & 0,00461 & * & & & i \\
\hline 237 & $-0,7178$ & i & * & i & & i & $-0,9116$ & I & $*$ & I & & 1 & 0,00453 & * & & & i \\
\hline 238 & 1,3863 & i & & i & * & I & 1,4644 & I & & I & * & I & 0,00437 & * & & & I \\
\hline 239 & $-0,5995$ & i & * & i & & i & $-0,7836$ & i & * & i & & i & 0,00803 & |* & & & i \\
\hline 240 & $-0,6157$ & i & * & i & & i & $-0,8017$ & i & * & i & & i & 0,00917 & * & & & i \\
\hline 241 & $-0,7260$ & i & $*$ & i & & i & $-0,9201$ & I & $*$ & i & & | & 0,0130 & * & & & i \\
\hline 242 & 1,1702 & i & & i & * & i & 1,3135 & i & & i & * & i & 0,0111 & 1 * & & & i \\
\hline 243 & $-0,5747$ & i & * & $i$ & & i & $-0,7555$ & i & * & i & & I & 0,0125 & * & & & i \\
\hline 244 & $-0,5977$ & $i$ & * & i & & i & $-0,7816$ & i & $*$ & i & & i & 0,00744 & |* & & & i \\
\hline 245 & $-0,8473$ & i & & i & & i & $-1,0403$ & i & $*$ & I & & I & 0,0141 & * & & & I \\
\hline 246 & $-0,6357$ & i & $*$ & i & & i & $-0,8239$ & i & $*$ & i & & i & 0,00993 & * & & & i \\
\hline 247 & 2,0097 & i & & i & & * i & 1,7984 & i & & i & * & $i$ & 0,00956 & | * & & & i \\
\hline 248 & $-0,7481$ & i & * & I & & i & $-0,9428$ & i & * & i & & 1 & 0,00460 & * & & & i \\
\hline 249 & $-0,7479$ & I & * & I & & I & $-0,9426$ & I & $*$ & i & & i & 0,00467 & |* & & & i \\
\hline 250 & $-0,6335$ & i & * & i & & i & $-0,8214$ & i & * & i & & I & 0,0371 & 1 & & & i \\
\hline 251 & $-0,6810$ & i & * & i & & i & $-0,8730$ & i & * & $i$ & & I & 0,0141 & * & & & i \\
\hline 252 & $-0,6160$ & i & $*$ & i & & i & $-0,8021$ & i & * & $i$ & & i & 0,00881 & * & & & i \\
\hline 253 & $-0,6493$ & i & * & i & & 1 & $-0,8387$ & 1 & $*$ & I & & I & 0,0150 & * & & & I \\
\hline 254 & 1,4596 & i & & i & * & i & 1,5108 & i & & i & $*$ & i & 0,00964 & |* & & & i \\
\hline 255 & 1,4829 & i & & i & * & i & 1,5250 & i & & i & * & 1 & 0,0103 & | * & & & i \\
\hline 256 & 1,8495 & i & & i & * & i & 1,7241 & I & & I & * & i & 0,00726 & $1^{*}$ & & & । \\
\hline 257 & $-0,5490$ & i & $*$ & & & i & $-0,7259$ & I & * & 1 & & 1 & 0,00687 & |* & & & i \\
\hline 258 & $-0,5239$ & i & * & i & & i & $-0,6965$ & i & $*$ & i & & i & 0,0100 & * & & & i \\
\hline
\end{tabular}


(continuação...)

Tabela 11: Análise de Resíduos do Conjunto de dados

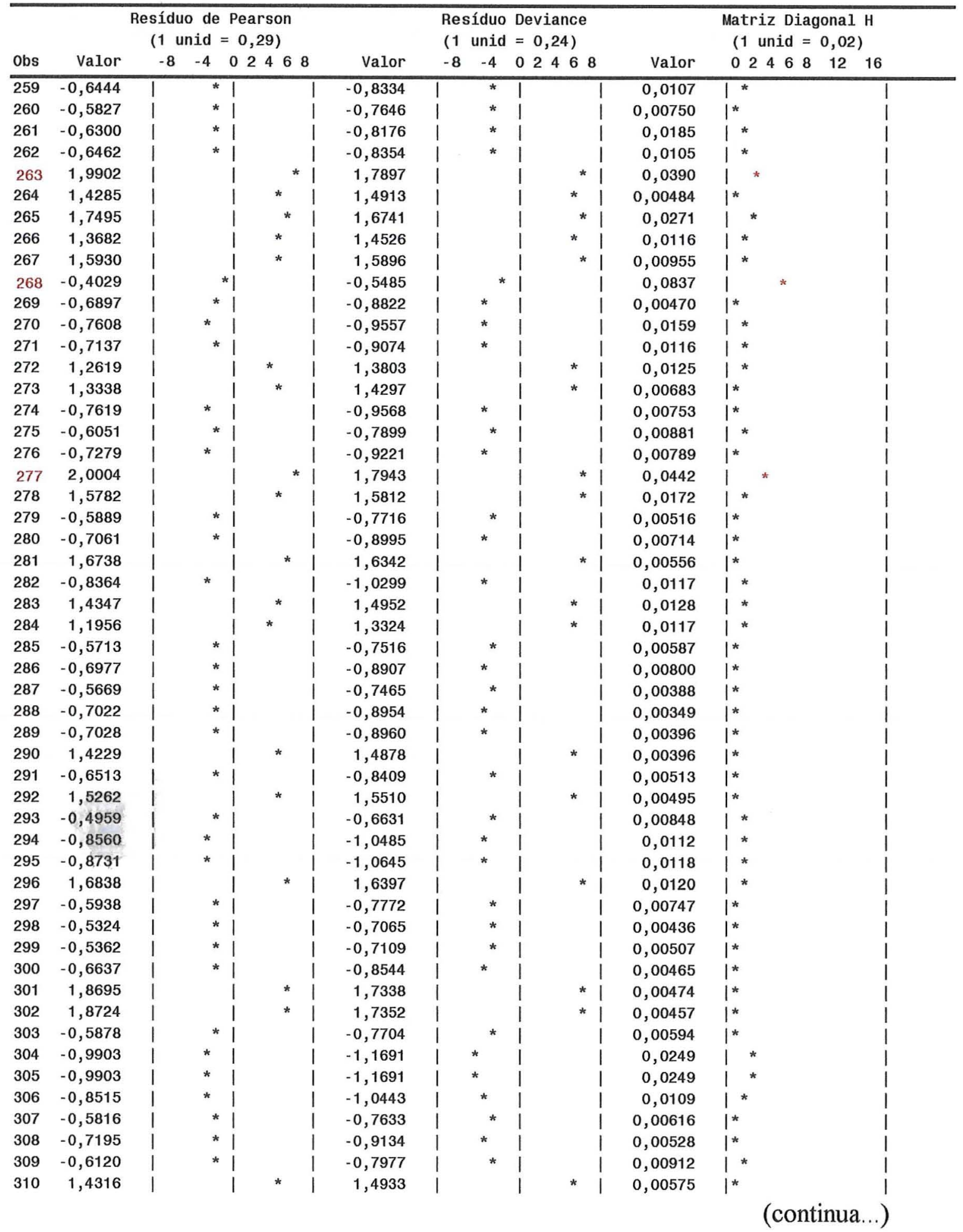


(continuação...)

Tabela 11: Análise de Resíduos do Conjunto de dados

\begin{tabular}{|c|c|c|c|c|c|c|c|c|c|c|c|c|c|c|c|c|}
\hline \multirow[b]{2}{*}{ Obs } & \multicolumn{7}{|c|}{$\begin{array}{c}\text { Resíduo de Pearson } \\
(1 \text { unid }=0,29)\end{array}$} & \multicolumn{5}{|c|}{$\begin{array}{l}\text { Resíduo Deviance } \\
(1 \text { unid }=0,24)\end{array}$} & \multicolumn{4}{|c|}{$\begin{array}{l}\text { Matriz Diagonal } \mathrm{H} \\
(1 \text { unid }=0,02)\end{array}$} \\
\hline & Valor & -8 & -4 & 024 & $46 \varepsilon$ & 8 & Valor & -8 & -4 & 0 & 468 & & Valor & 02468 & 12 & 16 \\
\hline 311 & $-0,9830$ & 1 & * & I & & 1 & $-1,1629$ & 1 & * & 1 & & 1 & 0,0599 & * & & $T$ \\
\hline 312 & $-0,6912$ & i & * & & & i & $-0,8837$ & i & * & i & & i & 0,00551 & * & & I \\
\hline 313 & 1,4582 & 1 & & i & * & i & 1,5099 & i & & i & * & i & 0,00578 & |* & & i \\
\hline 314 & $-0,5612$ & i & * & & & i & $-0,7400$ & i & * & i & & i & 0,00518 & * & & I \\
\hline 315 & $-0,8716$ & 1 & * & 1 & & i & $-1,0631$ & i & * & i & & i & 0,0624 & i & & i \\
\hline 316 & $-0,7241$ & I & * & & & I & $-0,9182$ & i & * & i & & | & 0,00624 & |* & & i \\
\hline 317 & $-0,6174$ & i & * & & & i & $-0,8036$ & i & * & i & & I & 0,00499 & * & & I \\
\hline 318 & $-0,7678$ & i & * & i & & i & $-0,9627$ & i & $*$ & i & & i & 0,00497 & * & & I \\
\hline 319 & 1,1669 & i & & | & * & I & 1,3110 & i & & i & * & 1 & 0,0220 & * & & 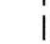 \\
\hline 320 & $-0,5296$ & I & * & & & I & $-0,7032$ & I & * & I & & I & 0,00474 & |* & & i \\
\hline 321 & $-0,6381$ & i & * & & & I & $-0,8266$ & i & * & i & & | & 0,0351 & 1 \% & & I \\
\hline 322 & $-0,7711$ & I & * & & & i & $-0,9660$ & i & * & i & & i & 0,0232 & i* & & i \\
\hline 323 & $-0,7345$ & i & * & i & & i & $-0,9290$ & i & * & i & & I & 0,0114 & * & & 1 \\
\hline 324 & 1,8379 & $i$ & & i & * & i & 1,7185 & i & & I & * & 1 & 0,0115 & * & & I \\
\hline 325 & $-0,6555$ & I & * & i & & i & $-0,8456$ & i & * & i & & i & 0,0129 & | * & & 1 \\
\hline 326 & $-0,8073$ & 1 & * & & & i & $-1,0018$ & i & * & i & & i & 0,0123 & i* & & i \\
\hline 327 & $-0,8073$ & i & * & & & i & $-1,0018$ & i & * & i & & i & 0,0123 & * & & I \\
\hline 328 & $-0,7553$ & i & * & i & & i & $-0,9501$ & i & * & i & & i & 0,00834 & i & & i \\
\hline 329 & $-0,6644$ & $i$ & * & i & & i & $-0,8551$ & i & * & i & & i & 0,00958 & | * & & i \\
\hline 330 & 1,8877 & 1 & & I & * & i & 1,7424 & i & & i & $*$ & i & 0,00813 & j* & & i \\
\hline 331 & $-0,5762$ & i & * & i & & i & $-0,7572$ & i & * & $i$ & & i & 0,00864 & * & & I \\
\hline 332 & $-0,5762$ & i & * & i & & i & $-0,7572$ & i & * & $i$ & & I & 0,00864 & i & & I \\
\hline 333 & $-0,8125$ & i & * & & & i & $-1,0069$ & i & * & i & & I & 0,00797 & * & & I \\
\hline 334 & $-0,7680$ & I & * & & & I & $-0,9629$ & i & * & i & & i & 0,0123 & i * & & i \\
\hline 335 & $-0,8097$ & I & * & 1 & & I & $-1,0041$ & I & * & I & & I & 0,00773 & $1^{*}$ & & I \\
\hline 336 & 1,2351 & i & & i & * & I & 1,3612 & 1 & & 1 & * & I & 0,00773 & $1^{*}$ & & I \\
\hline 337 & $-0,5794$ & 1 & * & & & i & $-0,7609$ & i & * & i & & i & 0,00631 & $i^{*}$ & & i \\
\hline 338 & $-0,6115$ & i & * & & & i & $-0,7971$ & i & * & i & & I & 0,0262 & 1 * & & I \\
\hline 339 & $-0,5860$ & i & * & & & I & $-0,7684$ & 1 & * & | & & | & 0,00615 & * & & I \\
\hline 340 & $-0,7097$ & i & * & & & i & $-0,9032$ & i & * & i & & i & 0,00711 & |* & & I \\
\hline 341 & 1, 3932 & i & & i & * & i & 1,4689 & i & & I & * & 1 & 0,00685 & * & & 1 \\
\hline 342 & $-0,6539$ & i & * & & & I & $-0,8438$ & 1 & * & i & & i & 0,0106 & * & & I \\
\hline 343 & 1,4491 & i & & i & * & I & 1,5043 & I & & i & * & 1 & 0,00711 & * & & I \\
\hline 344 & 1,5040 & i & & | & * & I & 1,5377 & 1 & & i & * & i & 0,00606 & * & & 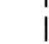 \\
\hline 345 & $-0,6440$ & i & * & & & i & $-0,8330$ & i & * & $i$ & & i & 0,0129 & i * & & i \\
\hline 346 & $-0,6440$ & $i$ & * & & & I & $-0,8330$ & I & * & i & & I & 0,0129 & * & & I \\
\hline 347 & $-0,6251$ & i & * & & & I & $-0,8122$ & I & * & i & & i & 0,00849 & * & & 1 \\
\hline 348 & $-0,6251$ & i & * & & & i & $-0,8122$ & i & * & i & & i & 0,00849 & 1 * & & i \\
\hline 349 & 1,6080 & 1 & & i & * & i & 1,5981 & i & & i & * & i & 0,00817 & i * & & i \\
\hline 350 & $-0,6406$ & i & * & i & & i & $-0,8292$ & 1 & * & i & & i & 0,00695 & $1^{*}$ & & I \\
\hline 351 & $-0,5722$ & i & * & i & & i & $-0,7526$ & 1 & * & I & & 1 & 0,0364 & 1 * & & \\
\hline 352 & $-0,7882$ & i & & & & I & $-0,9831$ & 1 & * & i & & I & 0,00667 & $1^{*}$ & & \\
\hline 353 & $-0,7829$ & i & * & & & I & $-0,9778$ & I & * & i & & i & 0,00640 & * & & \\
\hline 354 & 1,2687 & i & & 1 & * & I & 1,3851 & 1 & & | & * & i & 0,00667 & * & & \\
\hline 355 & 1,4148 & i & & i & * & i & 1,4827 & i & & i & * & i & 0,00740 & |* & & \\
\hline 356 & $-0,5771$ & i & * & i & & I & $-0,7582$ & i & * & i & & i & 0,00465 & * & & \\
\hline 357 & $-0,5763$ & i & * & & & I & $-0,7573$ & i & $*$ & i & & i & 0,00477 & * & & \\
\hline 358 & $-0,5754$ & i & * & & & i & $-0,7564$ & i & * & i & & i & 0,00493 & * & & \\
\hline 359 & $-0,5082$ & i & * & I & & | & $-0,6778$ & | & * & i & & | & 0,0387 & 1 * & & \\
\hline 360 & $-0,6908$ & i & * & i & & i & $-0,8834$ & i & * & i & & i & 0,00552 & $1^{*}$ & & \\
\hline 361 & $-0,5590$ & i & * & i & & I & $-0,7375$ & 1 & * & i & & | & 0,00612 & * & & \\
\hline 362 & $-0,5498$ & I & $*$ & i & & i & $-0,7268$ & i & $*$ & i & & i & 0,00646 & i* & & \\
\hline
\end{tabular}


(continuação...)

Tabela 11: Análise de Resíduos do Conjunto de Dados

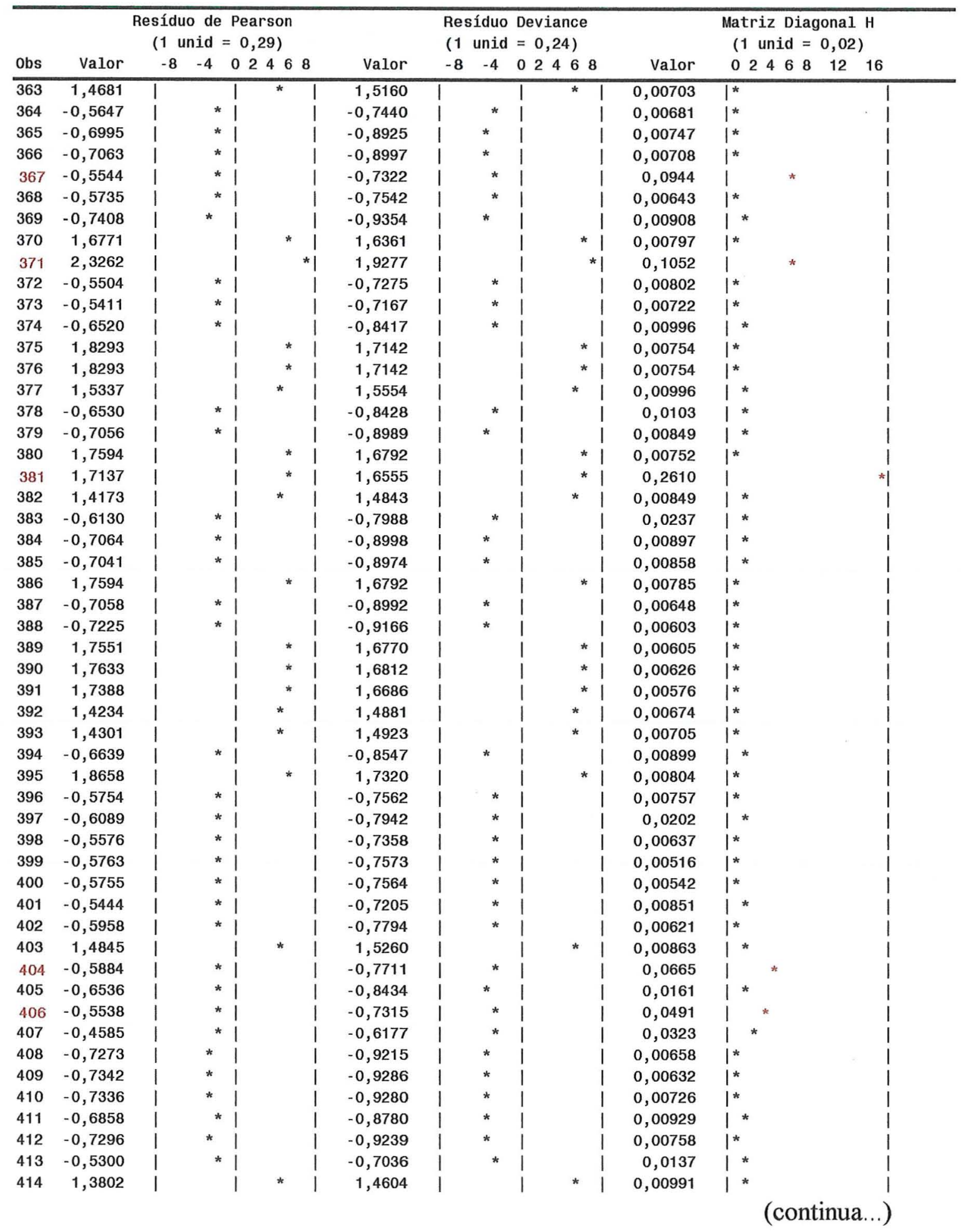


(continuação...)

Tabela 11: Análise de Resíduos do Conjunto de Dados

\begin{tabular}{|c|c|c|c|c|c|c|c|c|c|c|c|c|c|c|c|}
\hline \multirow[b]{2}{*}{ Obs } & \multicolumn{5}{|c|}{$\begin{array}{c}\text { Resíduo de Pearson } \\
(1 \text { unid }=0,29)\end{array}$} & \multicolumn{5}{|c|}{$\begin{array}{l}\text { Resíduo Deviance } \\
(1 \text { unid }=0,24)\end{array}$} & \multicolumn{5}{|c|}{$\begin{array}{l}\text { Matriz Diagonal } \mathrm{H} \\
(1 \text { unid }=0,02)\end{array}$} \\
\hline & Valor & -8 & -40 & 246 & 8 & Valor & -8 & -4 & 024 & 468 & Valor & & 2468 & 12 & 16 \\
\hline 415 & 1,3705 & $T$ & $T$ & * & $T$ & 1,4541 & $T$ & & $T$ & * & 0,0104 & 1 * & & & T \\
\hline 416 & 1,3802 & i & $i$ & * & i & 1,4604 & i & & | & * & 0,00991 & | * & & & i \\
\hline 417 & $-0,6964$ & i & * 1 & & i & $-0,8892$ & i & * & i & & 0,0122 & i * & & & i \\
\hline 418 & $-0,5539$ & I & * 1 & & i & $-0,7315$ & i & * & i & & 0,0355 & 1 & * & & i \\
\hline 419 & $-0,7289$ & 1 & * 1 & & i & $-0,9232$ & 1 & * & i & & 0,0150 & * & & & 1 \\
\hline 420 & $-0,2634$ & i & $* 1$ & & i & $-0,3662$ & i & * & $i$ & & 0,0791 & i & * & & i \\
\hline 421 & 1,7127 & I & i & * & i & 1,6550 & 1 & & 1 & * & 0,0180 & * & & & | \\
\hline 422 & $-0,6073$ & i & $* 1$ & & i & $-0,7924$ & I & * & i & & 0,00766 & |* & & & 1 \\
\hline 423 & 1,7755 & i & i & * & i & 1,6874 & i & & i & $*$ & 0,00388 & |* & & & 1 \\
\hline 424 & 1,3660 & | & I & * & | & 1,4511 & | & & 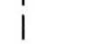 & $*$ & 0,00478 & * & & & 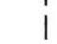 \\
\hline 425 & $-0,5689$ & i & $* 1$ & & i & $-0,7488$ & i & * & 1 & & 0,00396 & |* & & & 1 \\
\hline 426 & $-0,7224$ & i & $* 1$ & & | & $-0,9164$ & | & & I & & 0,00776 & * & & & 1 \\
\hline 427 & $-0,1467$ & i & $* 1$ & & i & $-0,2064$ & i & & *1 & & 0,0724 & i & * & & i \\
\hline 428 & 1,6277 & i & i & * & i & 1,6091 & i & & 1 & $\star$ & 0,00770 & * & & & I \\
\hline 429 & $-0,5525$ & 1 & $* 1$ & & | & $-0,7300$ & 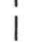 & * & 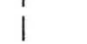 & & 0,00627 & * & & & 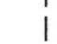 \\
\hline 430 & $-0,5445$ & i & $* i$ & & i & $-0,7207$ & i & * & i & & 0,00589 & |* & & & i \\
\hline 431 & $-0,6118$ & i & $* 1$ & & I & $-0,7975$ & I & * & 1 & & 0,0338 & 1 * & * & & I \\
\hline 432 & $-0,6065$ & i & * & & i & $-0,7915$ & 1 & * & 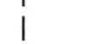 & & 0,00546 & * & & & 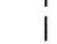 \\
\hline 433 & $-0,7391$ & i & * 1 & & i & $-0,9336$ & i & * & i & & 0,00573 & |* & & & I \\
\hline 434 & 1,7936 & | & I & * & i & 1,6965 & | & & | & * & 0,00738 & * & & & i \\
\hline 435 & $-0,6930$ & i & $* i$ & & I & $-0,8856$ & i & $*$ & i & & 0,00555 & $1^{*}$ & & & i \\
\hline 436 & $-0,7447$ & i & $* 1$ & & I & $-0,9393$ & | & $*$ & 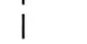 & & 0,00548 & $1^{*}$ & & & i \\
\hline 437 & 1,7498 & | & i & * & I & 1,6743 & i & & i & $*$ & 0,00649 & |* & & & i \\
\hline 438 & $-0,5472$ & i & $* 1$ & & I & $-0,7238$ & I & * & i & & 0,00477 & * & & & i \\
\hline 439 & 1,0612 & | & I & * & 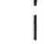 & 1,2283 & i & & 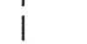 & * & 0,0841 & 1 & * & & 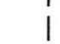 \\
\hline 440 & $-0,5954$ & i & $* i$ & & i & $-0,7790$ & i & * & i & & 0,00554 & $1^{*}$ & & & I \\
\hline 441 & 1,3557 & i & i & * & i & 1,4444 & | & & i & * & 0,00568 & $1^{*}$ & & & 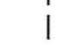 \\
\hline 442 & $-0,5891$ & i & $* 1$ & & I & $-0,7719$ & | & * & i & & 0,00556 & $1^{*}$ & & & i \\
\hline 443 & $-0,5214$ & i & $* 1$ & & I & $-0,6935$ & i & * & i & & 0,00507 & $1^{*}$ & & & 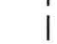 \\
\hline 444 & $-0,5996$ & | & * 1 & & I & $-0,7837$ & 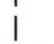 & * & 1 & & 0,0617 & 1 & * & & 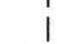 \\
\hline 445 & $-0,5643$ & i & * 1 & & i & $-0,7436$ & i & * & i & & 0,00535 & $1^{*}$ & & & I \\
\hline 446 & $-0,6915$ & i & $* 1$ & & I & $-0,8841$ & 1 & * & i & & 0,00611 & $1^{*}$ & & & 1 \\
\hline 447 & $-0,6940$ & i & * 1 & & | & $-0,8868$ & | & * & i & & 0,00590 & $j^{*}$ & & & i \\
\hline 448 & $-0,6439$ & i & $* 1$ & & 1 & $-0,8329$ & i & * & | & & 0,0108 & $1 *$ & & & I \\
\hline 449 & $-0,6627$ & 1 & $* 1$ & & i & $-0,8533$ & 1 & * & 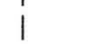 & & 0,00858 & * & & & 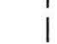 \\
\hline 450 & $-0,5397$ & i & * 1 & & 1 & $-0,7151$ & | & * & i & & 0,00672 & * & & & 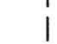 \\
\hline 451 & $-0,6825$ & i & * 1 & & i & $-0,8746$ & | & * & | & & 0,00715 & * & & & 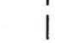 \\
\hline 452 & 1,8338 & i & 1 & * & I & 1,7165 & | & & 1 & * & 0,00678 & $1^{*}$ & & & 1 \\
\hline 453 & $-0,6061$ & i & * 1 & & 1 & $-0,7910$ & 1 & * & 1 & & 0,00853 & 1 * & & & i \\
\hline 454 & $-0,7650$ & i & * 1 & & 1 & $-0,9599$ & 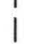 & * & 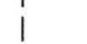 & & 0,00859 & * & & & 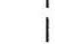 \\
\hline 455 & $-0,5088$ & i & * i & & i & $-0,6785$ & i & * & | & & 0,00673 & $1^{*}$ & & & I \\
\hline 456 & $-0,4943$ & i & $* 1$ & & I & $-0,6612$ & i & * & i & & 0,0169 & * & & & 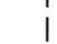 \\
\hline 457 & $-0,6088$ & i & $* 1$ & & 1 & $-0,7941$ & | & * & | & & 0,0136 & i * & & & I \\
\hline 458 & $-0,6207$ & i & * 1 & & I & $-0,8073$ & i & * & i & & 0,2013 & 1 & & * & I \\
\hline 459 & $-0,7034$ & i & * & & | & $-0,8966$ & 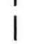 & * & 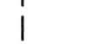 & & 0,00403 & * & & & 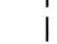 \\
\hline 460 & $-0,7029$ & i & * & & | & $-0,8961$ & i & * & 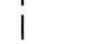 & & 0,00417 & |* & & & 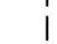 \\
\hline 461 & 1,2323 & i & i & * & 1 & 1,3592 & | & & 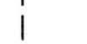 & * & 0,00712 & $1^{*}$ & & & 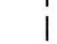 \\
\hline 462 & $-0,5146$ & i & * & & i & $-0,6855$ & 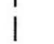 & * & 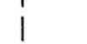 & & 0,00489 & * & & & 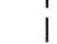 \\
\hline 463 & $-0,5172$ & i & * & & i & $-0,6886$ & i & * & i & & 0,00504 & $1^{*}$ & & & 1 \\
\hline 464 & $-0,5737$ & i & * & & i & $-0,7544$ & 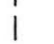 & * & 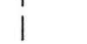 & & 0,0405 & 1 & * & & 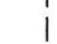 \\
\hline 465 & $-0,5578$ & i & * & & 1 & $-0,7360$ & | & * & i & & 0,00482 & * & & & 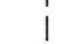 \\
\hline 466 & $-0,7121$ & i & $* 1$ & & | & $-0,9058$ & 1 & * & | & & 0,00596 & $1^{*}$ & & & i \\
\hline
\end{tabular}


(continuação...)

Tabela 11: Análise de Resíduos do Conjunto de Dados

\begin{tabular}{|c|c|c|c|c|c|c|c|c|c|c|c|c|c|c|c|c|}
\hline \multirow[b]{2}{*}{ Obs } & \multicolumn{6}{|c|}{$\begin{array}{c}\text { Resíduo de Pearson } \\
(1 \text { unid }=0,29)\end{array}$} & \multicolumn{5}{|c|}{$\begin{array}{l}\text { Resíduo Deviance } \\
(1 \text { unid }=0,24)\end{array}$} & \multicolumn{5}{|c|}{$\begin{array}{l}\text { Matriz Diagonal } \mathrm{H} \\
(1 \text { unid }=0,02)\end{array}$} \\
\hline & Valor & -8 & $\begin{array}{lll}-4 & 0 & 2\end{array}$ & 468 & & Valor & -8 & -4 & 02 & 468 & 8 & Valor & 0246 & 8 & 12 & 16 \\
\hline 467 & 1,9729 & 1 & 1 & * & & 1,7820 & 1 & & 1 & * & 1 & 0,00839 & $I^{*}$ & & & $\overline{1}$ \\
\hline 468 & $-0,6112$ & i & $* 1$ & & i & $-0,7967$ & i & $*$ & i & & i & 0,00869 & |* & & & i \\
\hline 469 & $-0,6495$ & 1 & $* 1$ & & i & $-0,8390$ & i & * & 1 & & I & 0,00741 & |* & & & i \\
\hline 470 & $-0,5692$ & i & $* i$ & & I & $-0,7493$ & i & * & i & & i & 0,00823 & | * & & & I \\
\hline 471 & 1,3615 & i & i & * & i & 1,4482 & i & & i & * & & 0,00433 & |* & & & 1 \\
\hline 472 & 1,3904 & i & i & * & i & 1,4670 & $i$ & & i & $*$ & i & 0,00902 & |* & & & i \\
\hline 473 & 1,6314 & I & I & * & I & 1,6111 & i & & i & $*$ & $i$ & 0,00880 & |* & & & I \\
\hline 474 & 1,4915 & i & i & * & i & 1,5302 & i & & | & $*$ & I & 0,00686 & * & & & I \\
\hline 475 & $-0,5602$ & I & * 1 & & I & $-0,7388$ & I & * & I & & 1 & 0,0116 & |* & & & i \\
\hline 476 & 1, 3194 & i & i & * & i & 1,4200 & i & & i & $*$ & & 0,0115 & 1 * & & & I \\
\hline 477 & 1,2972 & i & i & * & i & 1,4049 & i & & i & $*$ & I & 0,0113 & * & & & i \\
\hline 478 & $-0,6610$ & i & $* i$ & & i & $-0,8514$ & i & * & i & & i & 0,0122 & * & & & \\
\hline 479 & $-0,5316$ & i & * 1 & & i & $-0,7055$ & i & * & i & & i & 0,0109 & |* & & & \\
\hline 480 & $-0,5359$ & i & $* 1$ & & I & $-0,7106$ & i & * & i & & i & 0,00446 & * & & & \\
\hline 481 & $-0,5383$ & i & $* i$ & & i & $-0,7134$ & i & * & i & & i & 0,00428 & * & & & 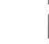 \\
\hline 482 & 1,4608 & i & i & * & i & 1,5115 & i & & i & * & i & 0,00570 & * & & & 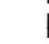 \\
\hline 483 & $-0,5352$ & I & $* i$ & & i & $-0,7098$ & i & * & i & & $i$ & 0,00493 & |* & & & 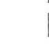 \\
\hline 484 & $-0,6575$ & i & $* 1$ & & I & $-0,8477$ & i & * & i & & i & 0,00492 & * & & & 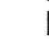 \\
\hline 485 & $-0,7053$ & 1 & $* 1$ & & I & $-0,8987$ & i & * & i & & i & 0,00635 & |* & & & 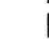 \\
\hline 486 & $-0,7348$ & i & * 1 & & i & $-0,9293$ & i & * & i & & i & 0,00414 & * & & & 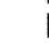 \\
\hline 487 & $-0,5606$ & i & $* 1$ & & i & $-0,7393$ & i & * & i & & i & 0,00491 & * & & & 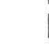 \\
\hline 488 & $-0,6503$ & i & $* i$ & & i & $-0,8398$ & i & * & i & & i & 0,00434 & |* & & & 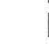 \\
\hline 489 & $-0,6369$ & i & $* 1$ & & i & $-0,8252$ & 1 & * & | & & I & 0,0286 & 1 * & & & \\
\hline 490 & $-0,7219$ & I & $* i$ & & i & $-0,9159$ & i & * & i & & i & 0,00655 & $1^{*}$ & & & 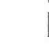 \\
\hline 491 & $-0,4108$ & i & *1 & & i & $-0,5585$ & i & * & 1 & & i & 0,0164 & * & & & t \\
\hline 492 & $-0,4361$ & I & ${ }^{*} \mid$ & & I & $-0,5901$ & I & * & i & & i & 0,0184 & i & & & 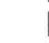 \\
\hline 493 & $-0,6880$ & i & $* i$ & & i & $-0,8804$ & i & * & i & & i & 0,0152 & |* & & & 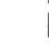 \\
\hline 494 & $-0,5822$ & 1 & * 1 & & I & $-0,7641$ & I & * & 1 & & i & 0,00445 & |* & & & 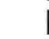 \\
\hline 495 & $-0,6556$ & i & $* i$ & & i & $-0,8457$ & i & * & i & & i & 0,00528 & |* & & & 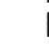 \\
\hline 496 & $-0,7761$ & i & * i & & i & $-0,9711$ & 1 & * & I & & i & 0,00528 & * & & & 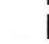 \\
\hline 497 & 1,2786 & 1 & I & * & i & 1,3920 & i & & i & $*$ & $i$ & 0,00543 & * & & & 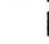 \\
\hline 498 & $-0,5715$ & i & $* 1$ & & i & $-0,7519$ & i & * & i & & I & 0,1452 & 1 & * & & 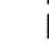 \\
\hline 499 & $-0,5474$ & 1 & * 1 & & 1 & $-0,7240$ & 1 & $*$ & 1 & & I & 0,0112 & 1* & & & \\
\hline 500 & $-0,5455$ & i & $* 1$ & & i & $-0,7218$ & i & * & i & & i & 0,0110 & $1 *$ & & & \\
\hline 501 & $-0,7103$ & i & $* i$ & & i & $-0,9038$ & i & * & I & & i & 0,00627 & $1^{*}$ & & & \\
\hline 502 & $-0,6939$ & 1 & $* 1$ & & i & $-0,8867$ & i & * & i & & i & 0,00575 & |* & & & \\
\hline 503 & $-0,6322$ & i & $* 1$ & & i & $-0,8201$ & i & * & $i$ & & i & 0,00602 & |* & & & \\
\hline 504 & 1,6970 & $i$ & $i$ & * & i & 1,6467 & i & & i & * & 1 & 0,00666 & |* & & & \\
\hline 505 & $-0,6815$ & i & $* 1$ & & i & $-0,8735$ & i & $*$ & i & & i & 0,00334 & |* & & & \\
\hline 506 & $-0,4925$ & 1 & * i & & i & $-0,6590$ & i & * & i & & i & 0,00687 & i* & & & \\
\hline 507 & $-1,0580$ & I & * $\quad 1$ & & i & $-1,2257$ & i & * & i & & i & 0,1375 & i & * & & \\
\hline 508 & $-0,7187$ & $i$ & * i & & i & $-0,9126$ & i & * & i & & i & 0,00474 & |* & & & \\
\hline 509 & $-0,5479$ & i & $* i$ & & i & $-0,7246$ & i & * & 1 & & I & 0,00445 & |* & & & \\
\hline 510 & 1,7999 & I & 1 & * & i & 1,6997 & i & & i & $*$ & $i$ & 0,00391 & |* & & & \\
\hline 511 & $-0,6706$ & i & $* i$ & & i & $-0,8618$ & i & * & i & & i & 0,00463 & * & & & \\
\hline 512 & 1,5015 & i & i & * & i & 1,5363 & i & & i & $*$ & i & 0,00382 & * & & & \\
\hline 513 & 1,0443 & i & i & * & i & 1,2144 & i & & i & * & $i$ & 0,1993 & i & & * & \\
\hline 514 & 1,3406 & 1 & 1 & * & I & 1,4343 & I & & 1 & * & I & 0,0149 & |* & & & \\
\hline 515 & $-0,3164$ & i & ${ }^{*} 1$ & & i & $-0,4368$ & i & * & i & & $i$ & 0,2086 & i & & * & * \\
\hline 516 & 1, 3296 & i & i & $*$ & i & 1,4270 & i & & 1 & $*$ & i & 0,0282 & 1 * & & & \\
\hline 517 & 1,6857 & i & i & * & i & 1,6406 & i & & i & $*$ & 1 & 0,00988 & | * & & & \\
\hline 518 & $-0,5185$ & i & $* i$ & & i & $-0,6901$ & $\mathrm{i}$ & $*$ & i & & $i$ & 0,0117 & | * & & & \\
\hline
\end{tabular}


(continuação...)

Tabela 11: Análise de Resíduos do Conjunto de Dados

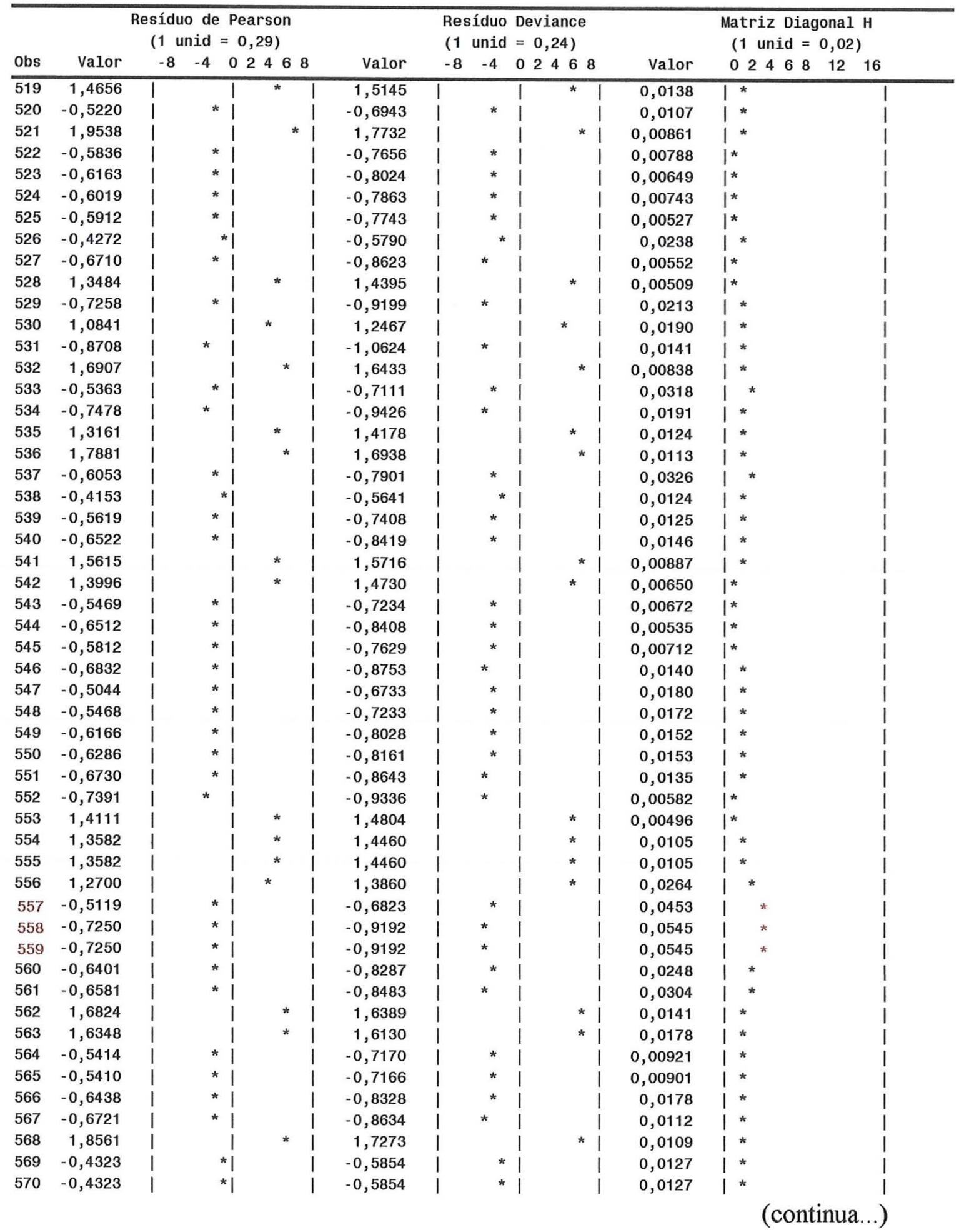


(continuação...)

Tabela 11: Análise de Resíduos do Conjunto de Dados

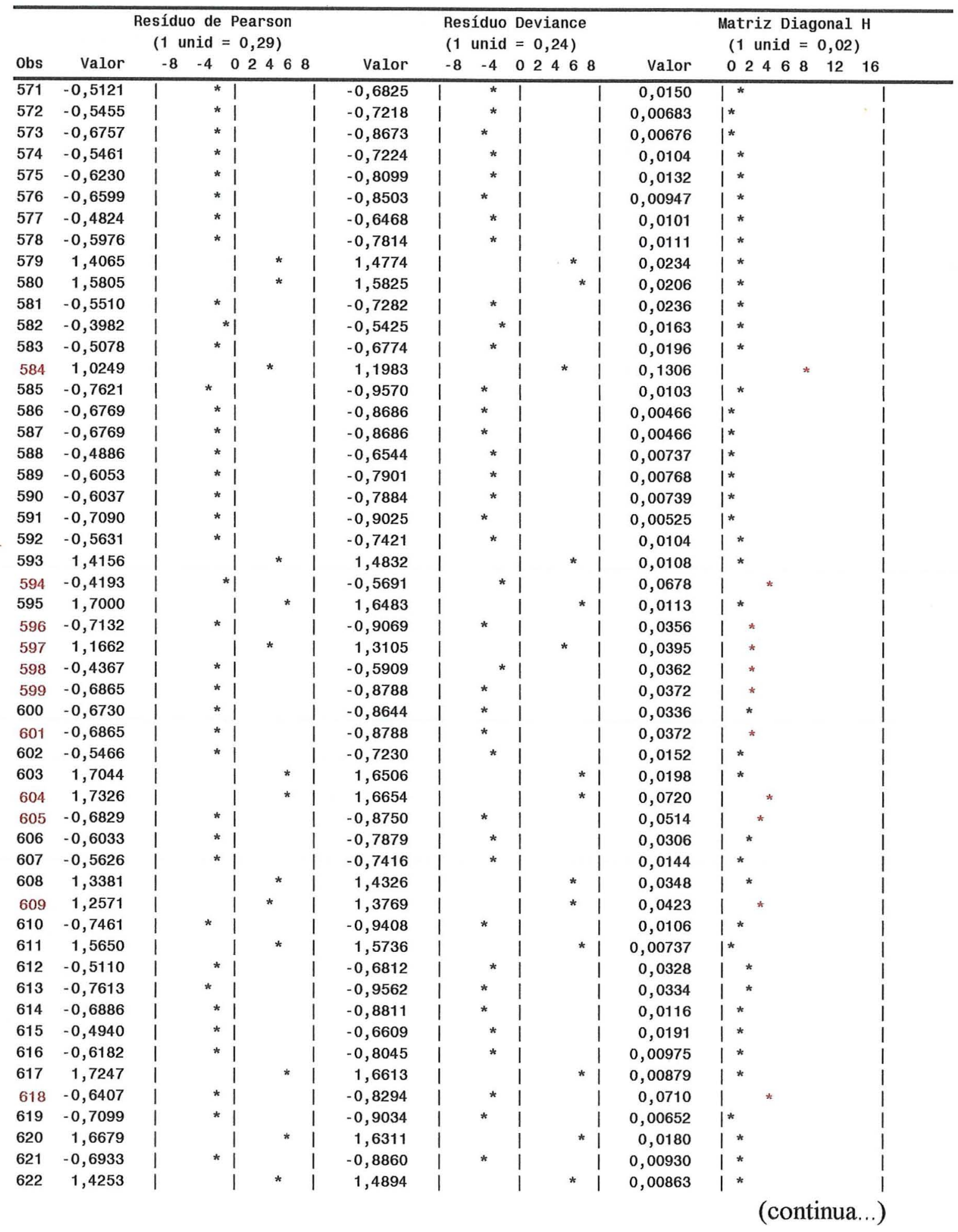


(continuação...)

Tabela 11: Análise de Resíduos do Conjunto de Dados

\begin{tabular}{|c|c|c|c|c|c|c|c|c|c|c|c|c|c|c|c|}
\hline \multirow[b]{2}{*}{ Obs } & \multicolumn{6}{|c|}{$\begin{array}{l}\text { Resíduo de Pearson } \\
(1 \text { unid }=0,29)\end{array}$} & \multicolumn{5}{|c|}{$\begin{array}{l}\text { Residuo Deviance } \\
(1 \text { unid }=0,24)\end{array}$} & \multicolumn{4}{|c|}{$\begin{array}{l}\text { Matriz Diagonal } H \\
(1 \text { unid }=0,02)\end{array}$} \\
\hline & valor & -8 & -4 & 02 & 46 & & Valor & -8 & -4 & 024 & 68 & Valor & 02468 & 12 & 16 \\
\hline$\overline{623}$ & $-0,6420$ & 1 & * & 1 & & 1 & $-0,8309$ & $T$ & * & 1 & 1 & 0,00618 & $T^{*}$ & & $T$ \\
\hline 624 & $-0,7426$ & 1 & * & 1 & & I & $-0,9372$ & 1 & * & 1 & 1 & 0,00931 & $1 *$ & & I \\
\hline 625 & 1,2665 & 1 & & 1 & * & 1 & 1,3835 & I & & 1 & $* 1$ & 0,00741 & $1^{*}$ & & I \\
\hline 626 & $-0,6113$ & i & & 1 & & i & $-0,7969$ & 1 & * & I & 1 & 0,0104 & $1 *$ & & I \\
\hline 627 & $-0,5399$ & i & * & 1 & & i & $-0,7153$ & i & * & i & 1 & 0,00629 & $1^{*}$ & & i \\
\hline 628 & 1,8573 & 1 & & 1 & * & l & 1,7279 & 1 & & 1 & $* 1$ & 0,00599 & $\left.\right|^{*}$ & & I \\
\hline 629 & 1,5119 & 1 & & 1 & $\star$ & I & 1,5425 & I & & I & * 1 & 0,00521 & $\left.\right|^{*}$ & & I \\
\hline 630 & $-0,5409$ & 1 & & 1 & & 1 & $-0,7164$ & 1 & * & I & I & 0,0106 & $1 *$ & & 1 \\
\hline 631 & $-0,6701$ & 1 & & i & & i & $-0,8612$ & $i$ & * & i & i & 0,0115 & $1 *$ & & i \\
\hline 632 & $-0,6218$ & i & & 1 & & i & $-0,8085$ & 1 & * & 1 & 1 & 0,0206 & * & & i \\
\hline 633 & $-0,6669$ & 1 & & * 1 & & I & $-0,8578$ & I & * & 1 & 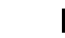 & 0,0198 & $1^{*}$ & & I \\
\hline 634 & $-0,5314$ & 1 & & 1 & & I & $-0,7053$ & i & * & 1 & 1 & 0,00956 & $1 *$ & & i \\
\hline 635 & $-0,8514$ & 1 & * & $i$ & & i & $-1,0441$ & 1 & * & 1 & 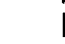 & 0,0296 & $1 *$ & & i \\
\hline 636 & 1,5189 & i & & i & * & i & 1,5467 & i & & i & $* 1$ & 0,0106 & I* & & i \\
\hline 637 & 1,5189 & 1 & & I & * & i & 1,5467 & 1 & & i & $* 1$ & 0,0106 & * & & $i$ \\
\hline 638 & 1,2931 & 1 & & i & * & i & 1,4020 & i & & i & * & 0,0128 & 1* & & i \\
\hline 639 & $-0,7433$ & i & * & 1 & & i & $-0,9380$ & 1 & * & 1 & i & 0,00599 & $1^{*}$ & & i \\
\hline 640 & 1,3401 & $i$ & & $i$ & * & i & 1,4340 & i & & i & $* 1$ & 0,00631 & $1^{*}$ & & i \\
\hline 641 & 1,3401 & i & & i & * & i & 1,4340 & 1 & & i & $* 1$ & 0,00631 & 1* & & i \\
\hline
\end{tabular}


Gráfico 1 - Gráfico dos Resíduos de Pearson
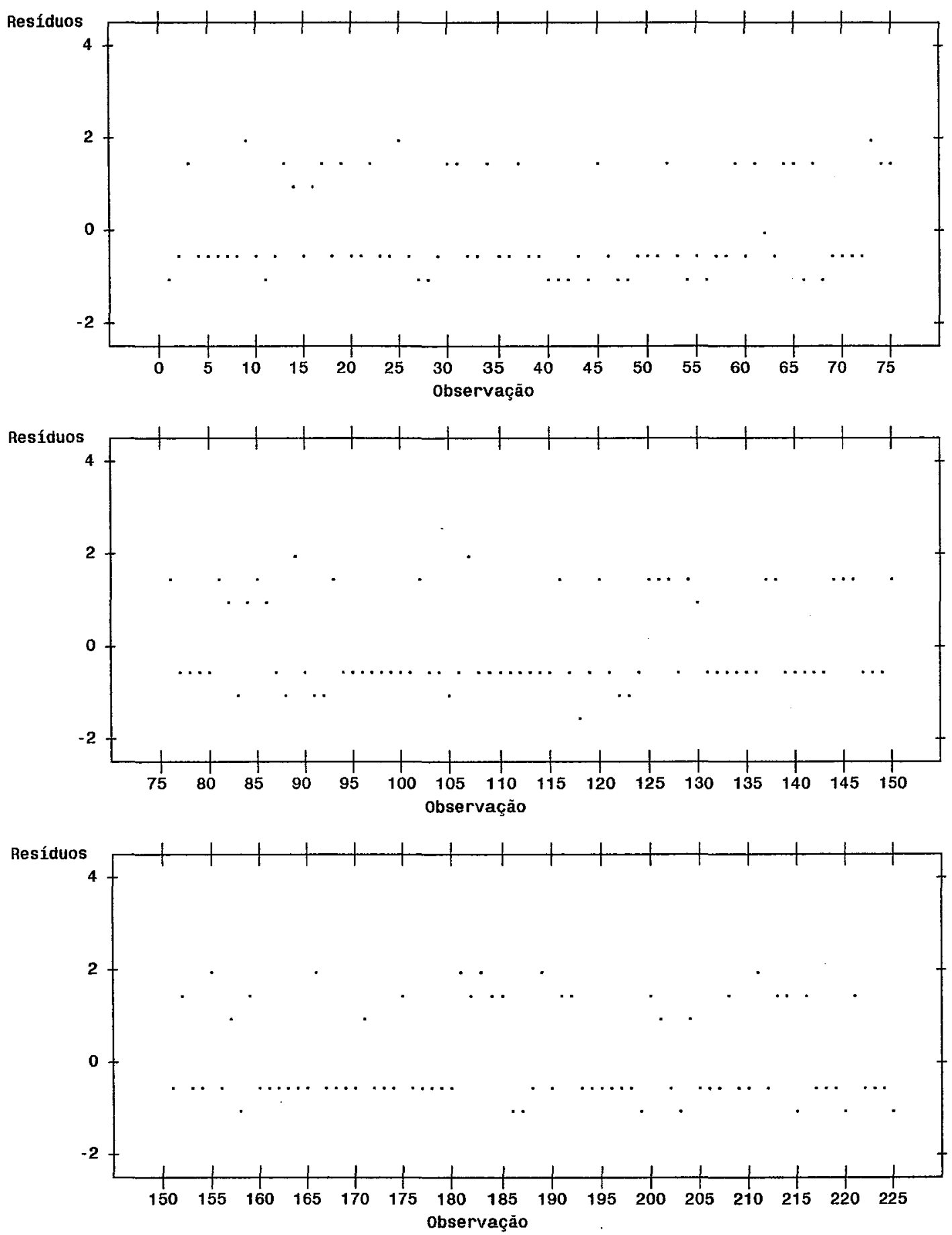

(continua ...) 
(continuação...)

Gráfico 1 - Gráfico dos Resíduos de Pearson
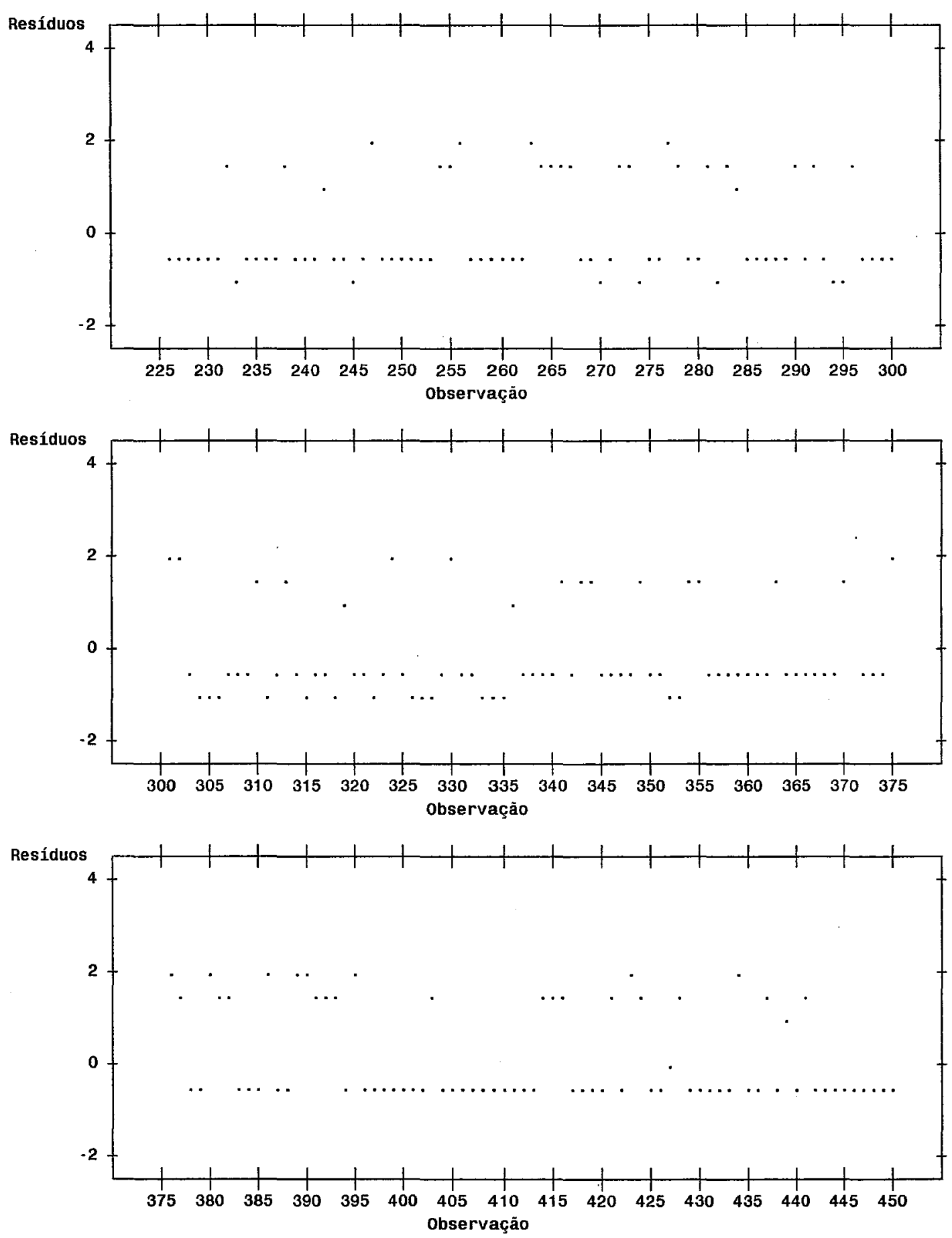

(continua...) 
(continuação...)

Gráfico 1 - Gráfico dos Resíduos de Pearson
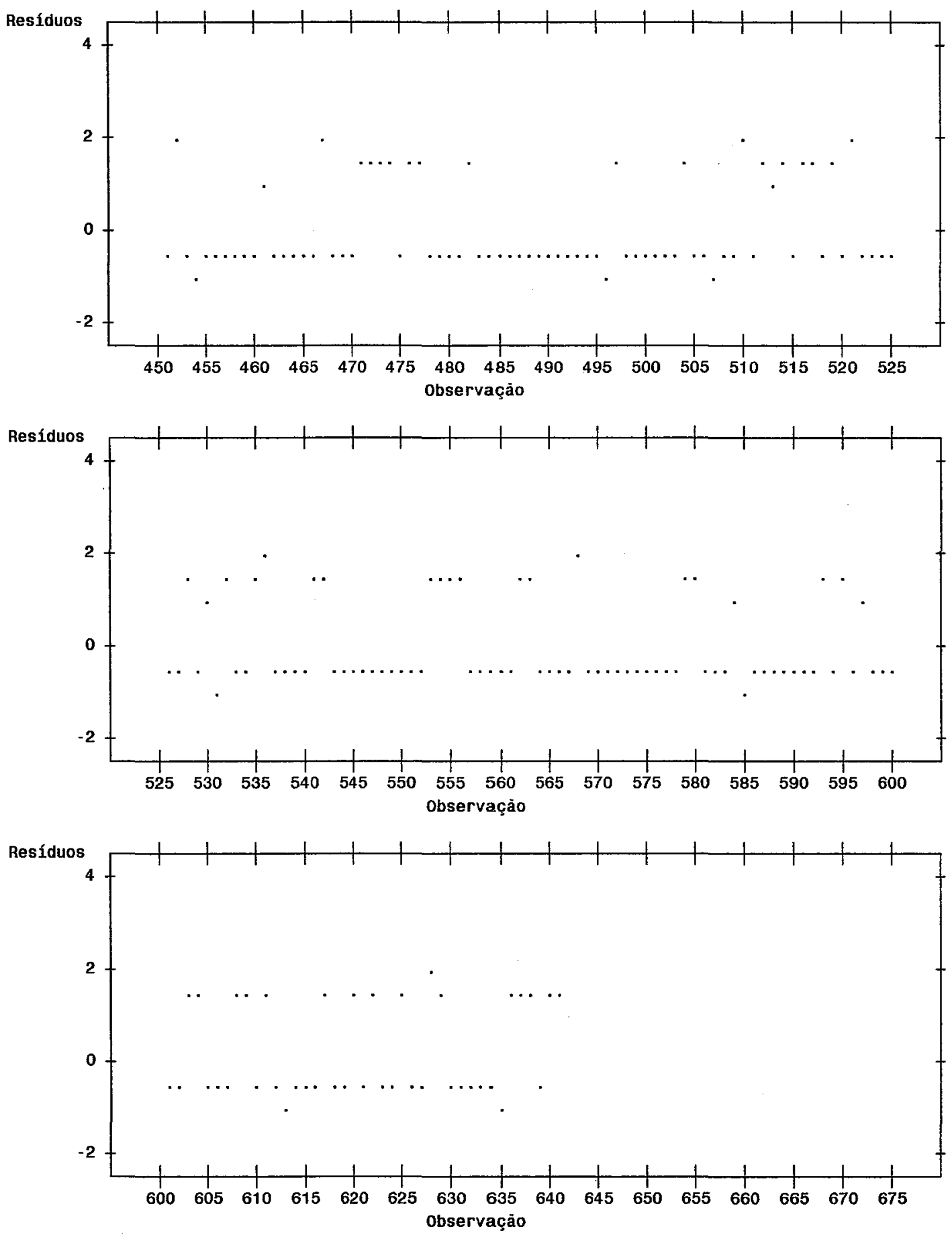
Gráfico 2- Gráfico dos Resíduos Deviance
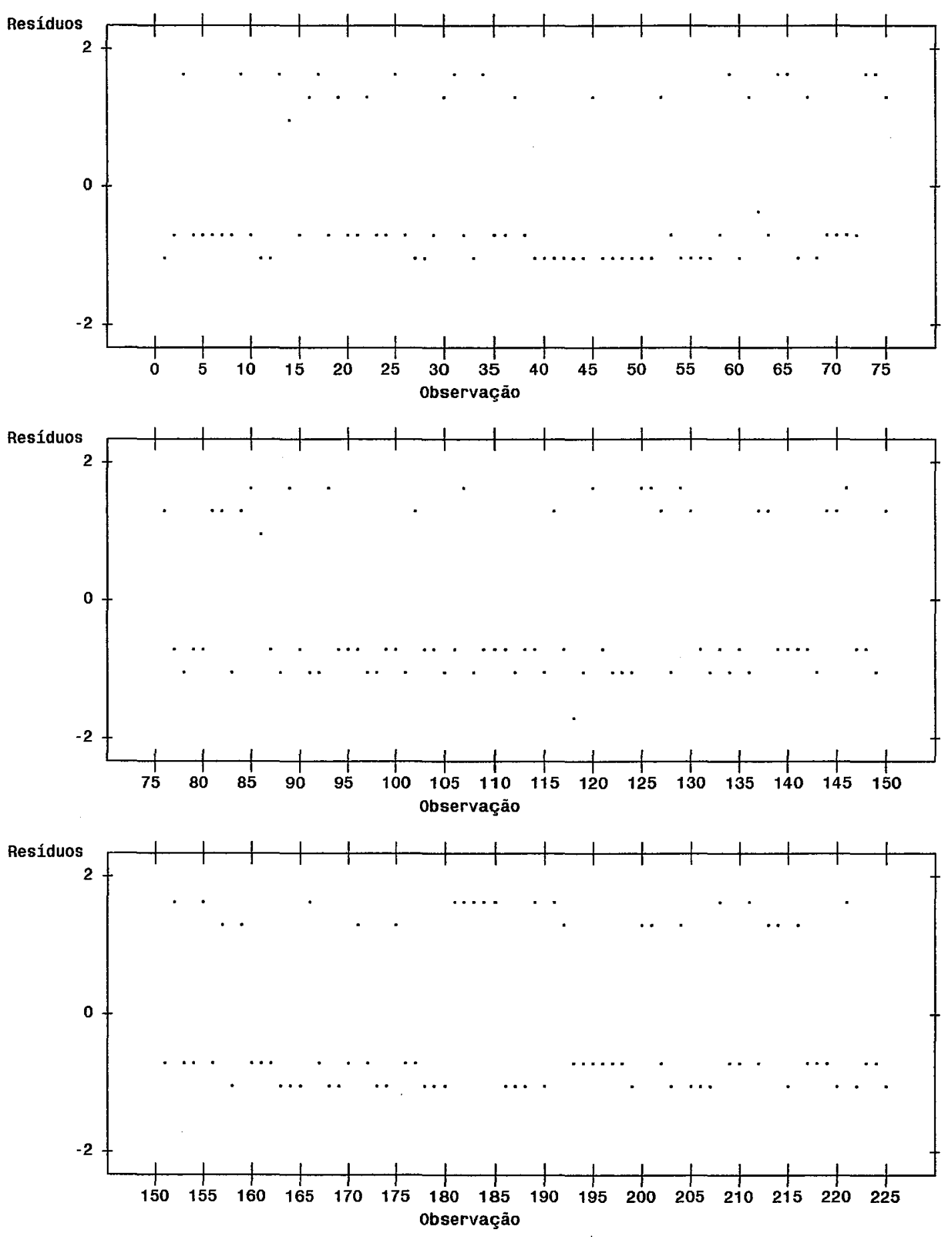

(continua...) 
(continuação...

Gráfico 2- Gráfico dos Resíduos Deviance
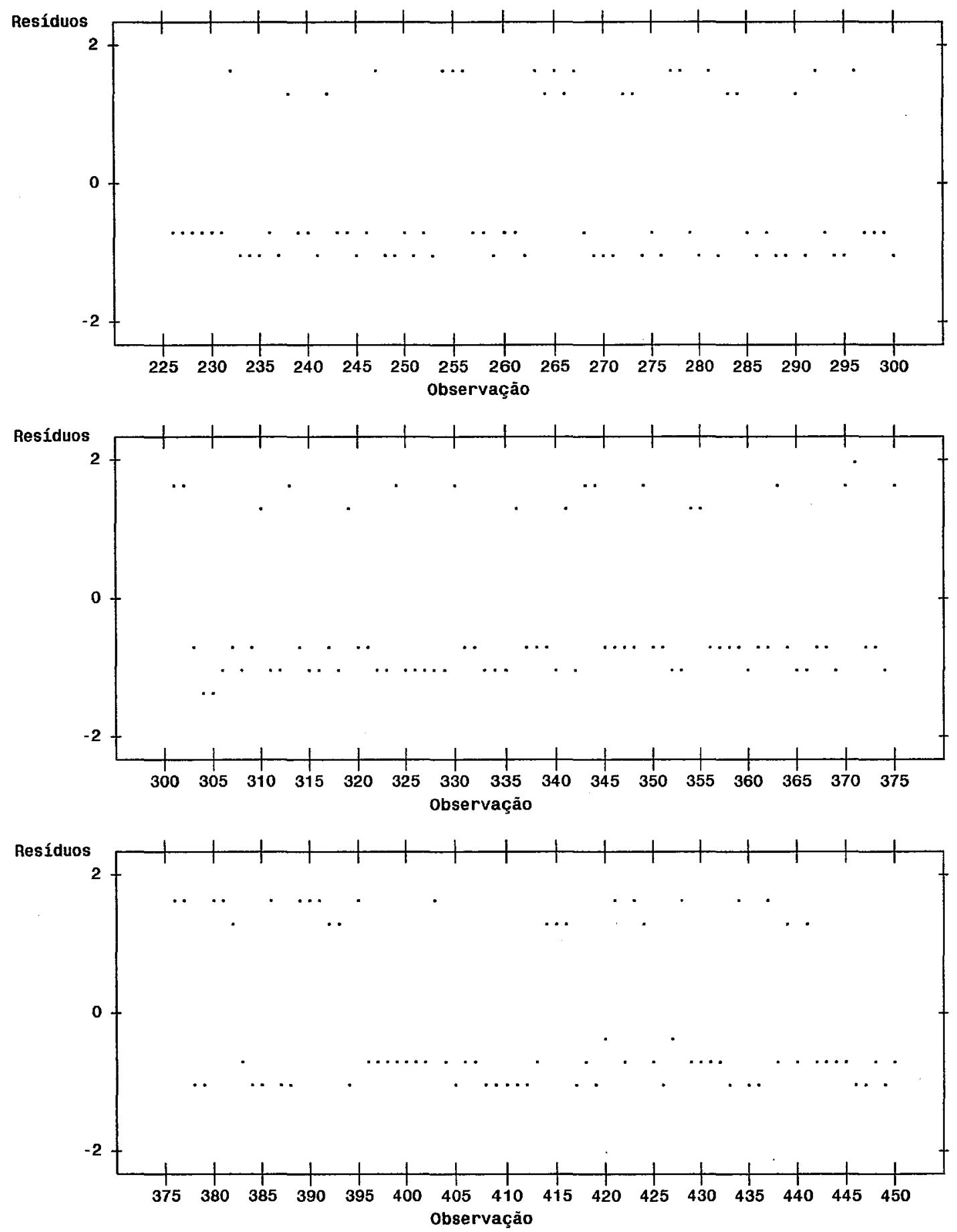

(continua...) 
(continuação...

Gráfico 2- Gráfico dos Resíduos Deviance
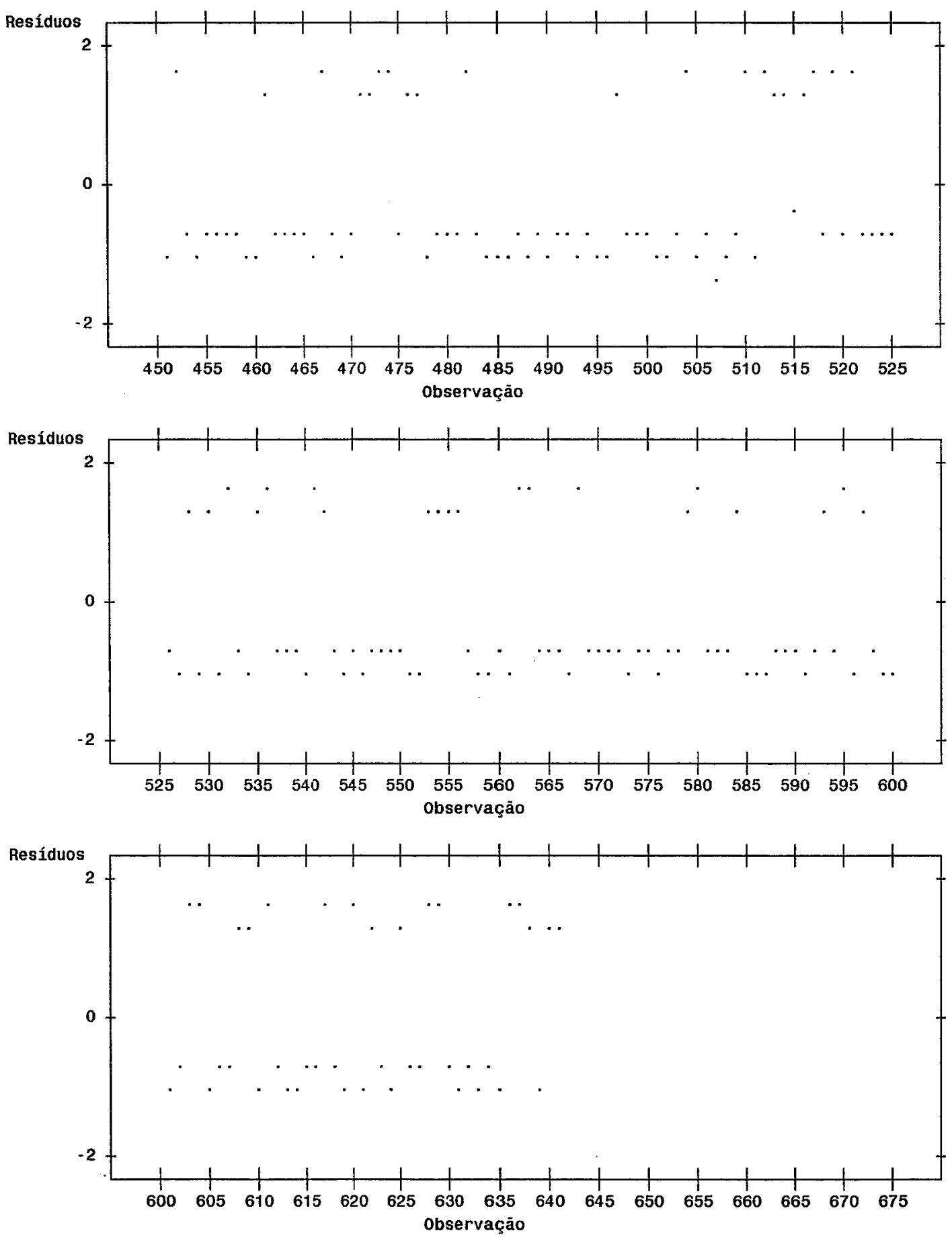
Gráfico 3 - Gráfico da Matriz Diagonal H
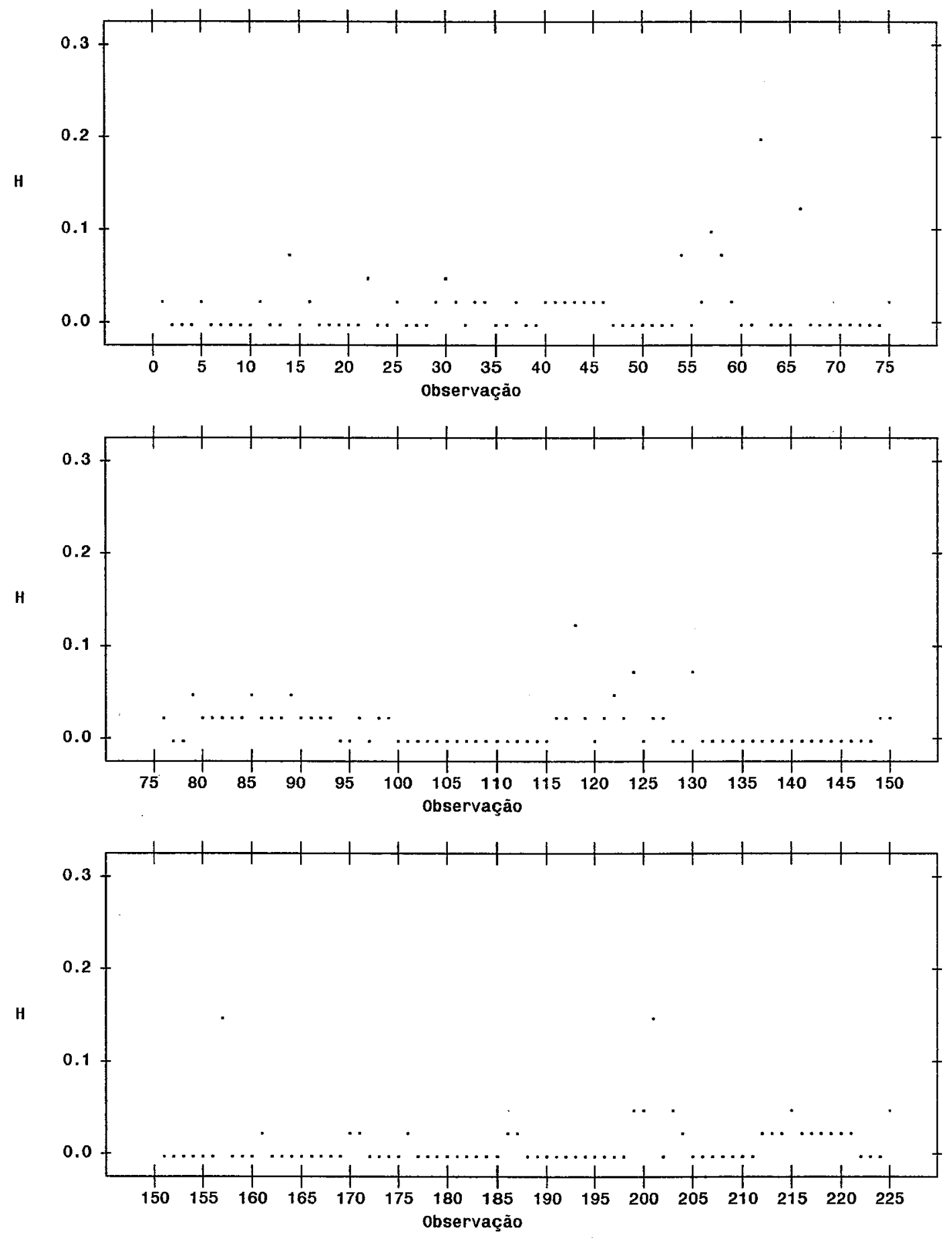

(continua...) 
(continuação...)

Gráfico 3 - Gráfico da Matriz Diagonal H

H
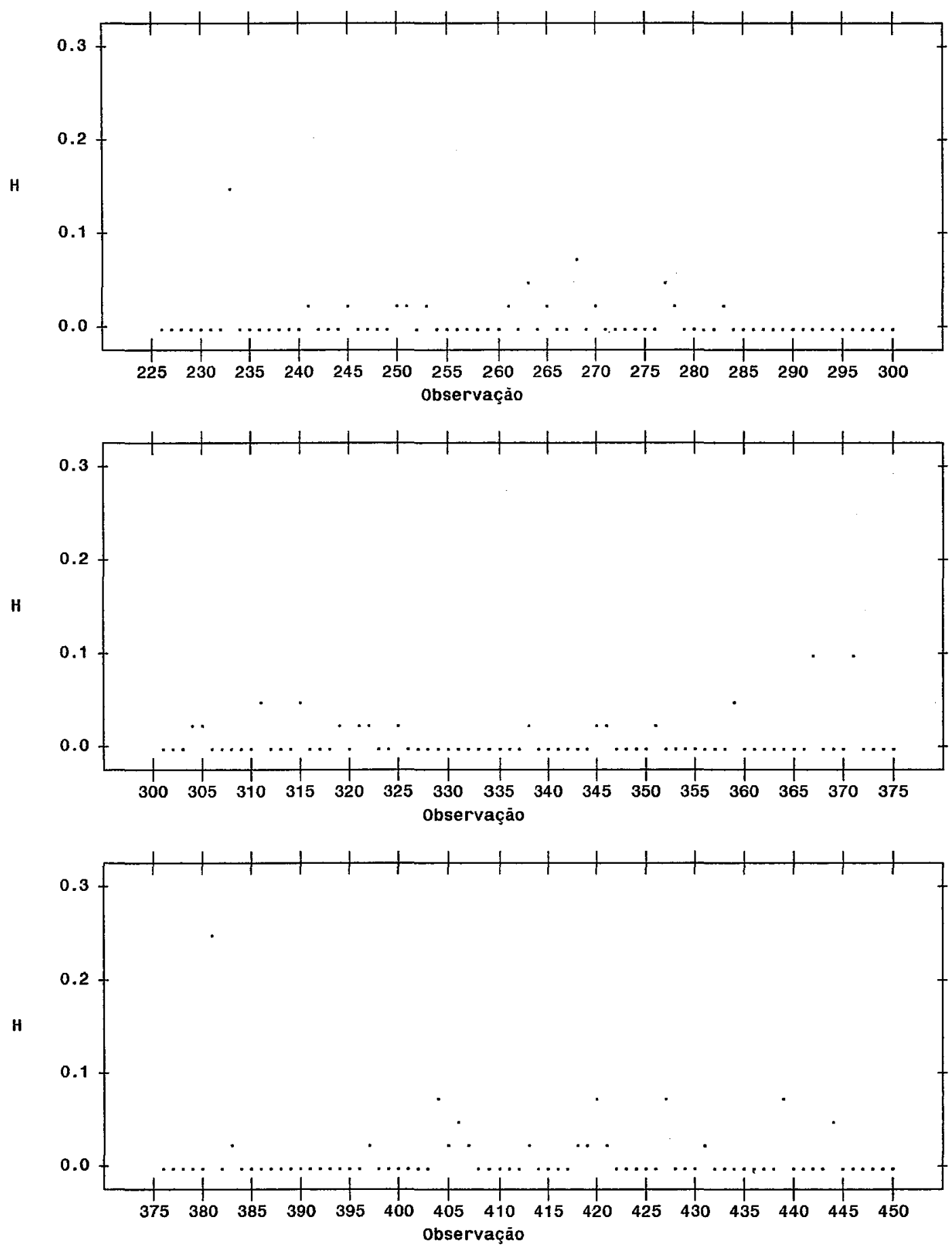

(continua...) 
(continuação...)

Gráfico 3 - Gráfico da Matriz Diagonal H

H

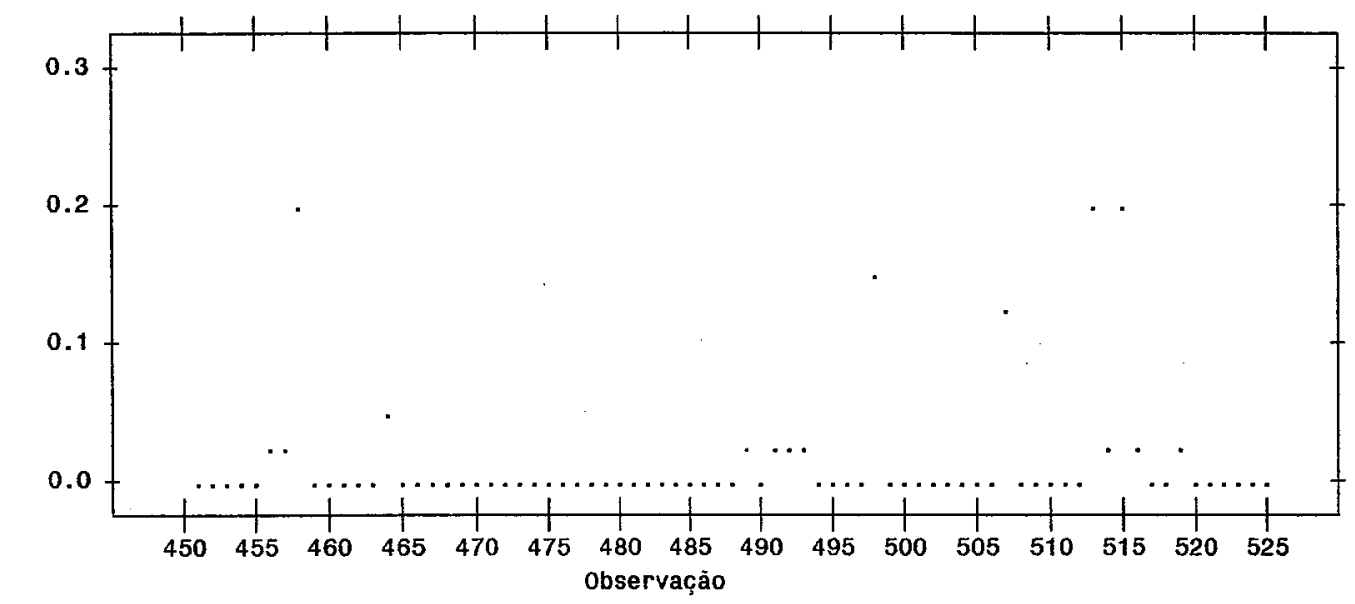

H
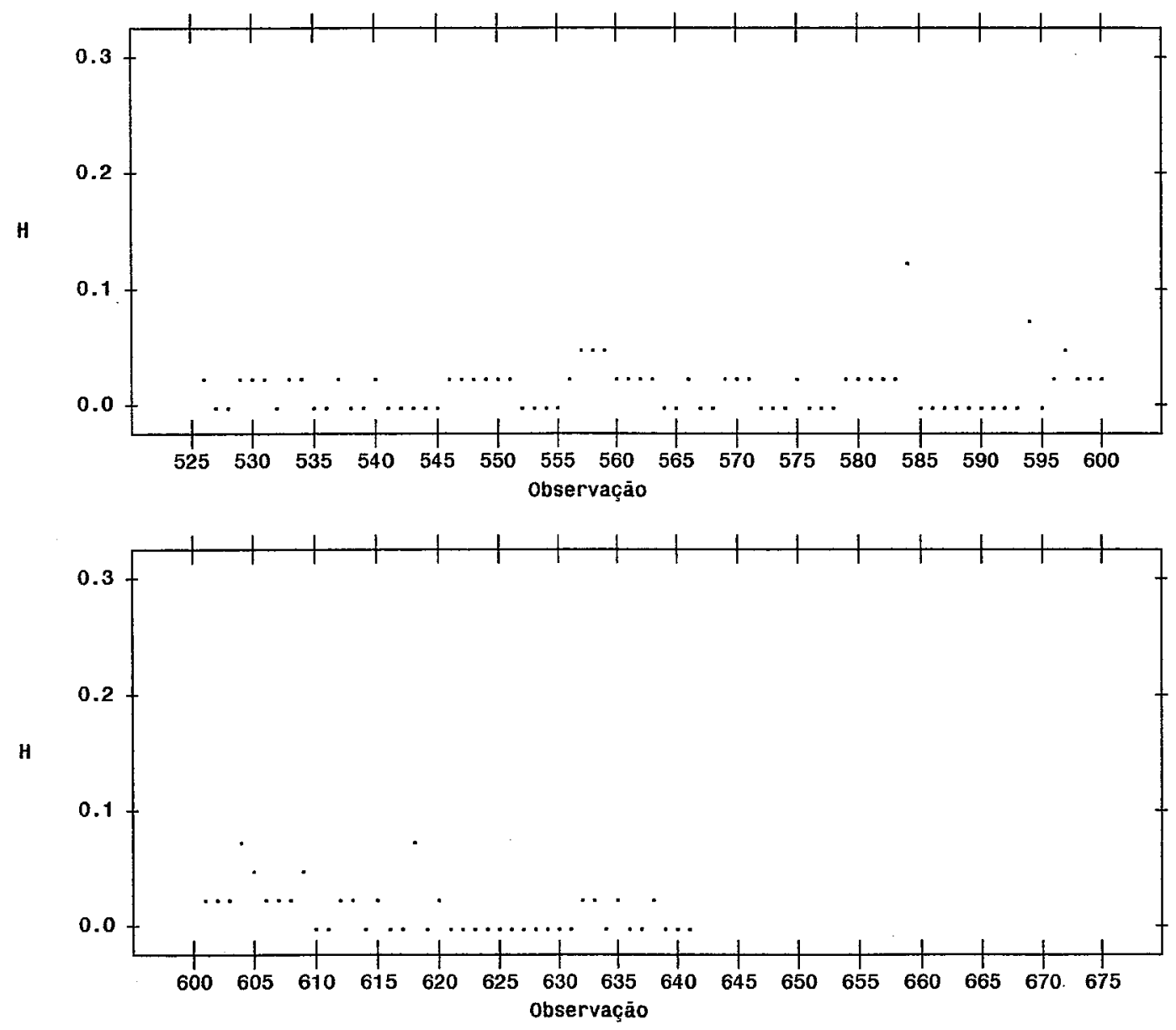